\title{
An experimentalist's guide to the matrix element in angle resolved photoemission
}

\author{
Simon Moser \\ Institute of Condensed Matter Physics, \\ Ecole Polytechnique Fédérale de Lausanne (EPFL), \\ CH-1015 Lausanne, \\ Switzerland \\ Advanced Light Source (ALS), \\ Berkeley, California 94720, \\ USA
}

(Dated: November 8, 2016)

\begin{abstract}
Angle resolved photoemission spectroscopy (ARPES) is commonly known as a powerful probe of the one-electron removal spectral function in ordered solid state. With increasing efficiency of light sources and spectrometers, experiments over a wide range of emission angles become more and more common. Consequently, the angular variation of ARPES spectral weight - often times termed "matrix element effect" - enters as an additional source of information. In this tutorial, we develop a simple but instructive free electron final state approach based on the three-step model to describe the intensity distribution in ARPES. We find a compact expression showing that the ARPES spectral weight of a given Bloch band is essentially determined by the momentum distribution (the Fourier transform) of its associated Wannier orbital - times a polarization dependent pre-factor. While the former is giving direct information on the symmetry and shape of the electronic wave function, the latter can give rise to surprising geometric effects. We discuss a variety of modern and instructive experimental showcases for which this simplistic formalism works astonishingly well and discuss the limits of this approach.
\end{abstract}

Keywords: ARPES, intensity distribution, matrix elements, dichroism, Fourier transform, tight binding

\section{CONTENTS}

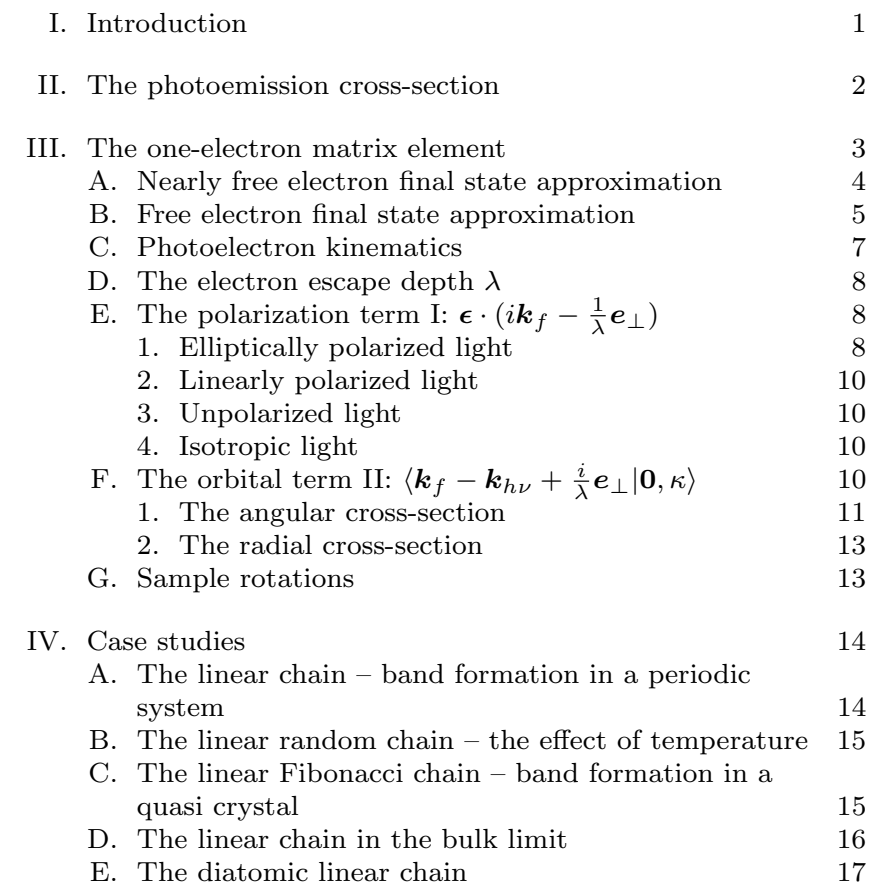

* E-mail address: skmoser@lbl.gov
F. Orbital mapping of ordered molecules $\quad 18$ G. Matrix elements in graphene 20 H. Matrix elements and spin orbit coupling 21 \begin{tabular}{|l|l|}
\hline I. Matrix elements in multilayer systems & 22 \\
\hline
\end{tabular} J. Matrix elements in doped oxides 23 K. Matrix elements in cuprates 24

V. Concluding remarks 26

\begin{tabular}{|ll}
\hline VI. Acknowledgements & 27
\end{tabular}

VII. Appendix: rotation of atomic orbitals 27

References 28

\section{INTRODUCTION}

Photoemission spectroscopy is an old and well established technique, dating back to the discovery of the photoelectric effect by Hertz (1887), Hallwachs (1888) and Lenard (1900) at the end of the 19th century and its explanation by Einstein (1905). Half a century later, Kai Siegbahn transformed the effect into a usable experimental technique which has been continuously developed further (Nordling et al. 1957). Many of the theoretical concepts and models used to interpret our today's experimental observations therefore have been developed many years ago and are summarized in reviews and textbooks Braun, 1996, Brundle and Baker, 1978, Carlson, 1975; 
Damascelli et al., 2003, Fadley, 2010; Himpsel, 1983 , Hüfner, 1995, 2007, Kevan, 1992, Plummer and Eberhardt, 1982, Turner, 1988).

Today, angle resolved photoemission spectroscopy (ARPES) experiments are performed routinely on solid state surfaces. With the advent of 3rd generation synchrotrons, improvements in resolution and detectors, it became possible to sample large volumes in energy and momentum space as a function of the photon polarization with very high throughput. Recent advances in detector technology are promising to further simultaneously add the spatial (Escher et al. 2005, Krömker et al. 2008), the spin (Tusche et al. | 2011; Vasilyev et al.|| 2015), and time (Bovensiepen and Kirchmann, 2012 ; Smallwood et al. 2012 Stolow et al. . 2004) degrees of freedom in an efficient manner. This typically implies that the experimentalist returns from the experiment with a vast amount of multidimensional data (Schoenhense et al. 2015$)$.

The main information extracted from an ARPES dataset is usually the band structure and its associated one-electron removal spectral function, which directly relates to the many body effects in the system. Typically it is overlooked however that ARPES provides additional information about the electronic system. One such example is the variation of ARPES intensity throughout three dimensional $\boldsymbol{k}$-space, often times dubbed "matrix element effects". Usually, these effects are considered a drawback rather than an asset, as they can be confused with many body interactions (Inosov et al., 2007), and are often times accounted for by hand-waving arguments.

More recently, a new branch of photoemission has started to focus on the spectral weight distribution of adsorbed molecules, which contains information about the real space distribution of electrons associated with a certain molecular state. The underlying experimental goal is to selectively map the molecular wave function, and the technique is typically referred to as "orbital tomography" (Berkebile et al., 2008, Koller et al., 2007, Puschnig et al., 2009). Another branch of photoemission focuses on spin orbit coupled systems, in which the spin- is locked to the orbital degrees of freedom. Photoemission, which primarily affects the electronic part of the wave function, is used here to indirectly probe the spin texture and again, the underlying mechanism is encoded in the intensity distribution of the photoelectrons (Jozwiak et al., 2013, Park and Louie, 2012, Zhu et al. 2013).

The theoretical concept behind many of these ideas however - i.e. a fully relativistic many body treatment of the photoemission cross-section - is somewhat complicated and hides the intuitive physical origin of many of these effects. It is therefore useful to return and consistently rebuild some simpler concepts that were already developed during the "early days" of photoemission.

This tutorial aims at consistently introducing the most important concepts of ARPES and its transition matrix element. It is guided by the free electron final state approximation in the three-step model, which can easily be generalized by the ambitious reader. We will give enough details as needed for a comprehensive understanding of the photoemission spectral weight, but staying concise enough to provide a useful "pencil and paper" toolset for the experimentalist on-the-job. The tutorial is organized as follows:

In Sec. II we review the photoemission cross-section derived from first order perturbation theory. We discuss all approximations necessary to separate the single electron transition matrix element from the one-electron removal spectral function.

In Sec. III, we derive a closed form expression for the transition matrix element in the nearly free electron final state approximation. For everyday use, we restrict the final state to one single plane wave component, mimicking its evanescent character inside the solid. We derive that the photoemission intensity essentially goes as the Fourier transform of the Wannier orbital associated to the probed Bloch band, times a polarization dependent pre-factor. In the tight binding approximation, the sooner takes a particularly simple form reflecting the local orbital symmetry. The latter turns out to be at the origin of surprising effects, like geometrical circular dichroism. Finally, we discuss how the photoemission cross-section may be influenced by sample rotations during the ARPES experiment.

In Sec. IV we turn to some instructive showcases of modern ARPES. We start from the concept of the linear chain and discuss band formation in finite and infinite systems. We then focus on some recent results for ordered organic molecules. These concepts are then expanded to two dimensional infinite systems like graphene and topological surface states. At the end of this tutorial, we will construct a simple model to explain the intensity variation in two complex oxides and finally discuss the limitations of our approach in Sec. V

\section{THE PHOTOEMISSION CROSS-SECTION}

Photoemission measures the transition probability $w_{f i}$ for an optical excitation between an $N$-electron ground state $\left|\Psi_{i}^{N}\right\rangle$ and one of the available final states $\left|\Psi_{f}^{N}\right\rangle$ with one photoelectron (Armitage et al., 2003 , Damascelli, 2004, Damascelli et al., 2003; Hüfner, 1995, 2007; Plummer and Eberhardt, 1982). Assuming the interaction between incoming light and matter to be weak, this process can be conveniently described by Fermi's Golden rule for first order processes

$$
w_{f i}=\frac{2 \pi}{\hbar}\left|\left\langle\Psi_{f}^{N}\left|H_{i n t}\right| \Psi_{i}^{N}\right\rangle\right|^{2} \delta\left(E_{f}^{N}-E_{i}^{N}-h \nu\right),
$$

where $E_{i}^{N}$ and $E_{f}^{N}$ are the initial and final state energies of the $N$-particle system, respectively, and $h \nu$ is the 
photon energy.

In the non relativistic limit, the light matter interaction can be treated as incoming light. In second quantization, this Hamiltonian can be expressed as

$$
H_{i n t}=\sum_{\boldsymbol{k}_{f}, \boldsymbol{k}} M_{\boldsymbol{k}_{f} \boldsymbol{k}} \hat{c}_{\boldsymbol{k}_{f}}^{\dagger} \hat{c}_{\boldsymbol{k}}
$$

$H_{\text {int }}=\sum_{i=1}^{N}\left[\frac{e}{m} \boldsymbol{A}\left(\boldsymbol{r}_{i}\right) \cdot \hat{\boldsymbol{p}}_{i}+\frac{e^{2}}{2 m} \boldsymbol{A}^{2}\left(\boldsymbol{r}_{i}\right)+\frac{e \hbar}{2 m} \hat{\boldsymbol{\sigma}}_{i} \cdot \nabla \times \boldsymbol{A}\left(\boldsymbol{r}_{i}\right)\right.$

$$
\left.-\frac{e^{2} \hbar}{(2 m c)^{2}} \hat{\boldsymbol{\sigma}}_{i} \cdot \frac{\partial \boldsymbol{A}_{i}\left(\boldsymbol{r}_{i}\right)}{\partial t} \times \boldsymbol{A}\left(\boldsymbol{r}_{i}\right)\right]
$$

where $\hat{\boldsymbol{p}}_{i}$ is the momentum operator and $\boldsymbol{r}_{i}$ the position of electron $i$. The $\hat{\boldsymbol{\sigma}}_{i}$ are the Pauli spin matrices. Neglecting the spin dependent parts and the terms quadratic in the vector potential $\boldsymbol{A}$, we remain with

$$
\begin{aligned}
H_{i n t} & \sim \frac{e}{m} \sum_{i=1}^{N} \boldsymbol{A}\left(\boldsymbol{r}_{i}\right) \cdot \hat{\boldsymbol{p}}_{i}=\frac{e}{m c} \sum_{i=1}^{N} e^{i \boldsymbol{k}_{h \nu} \cdot \boldsymbol{r}_{i}} \boldsymbol{\epsilon} \cdot \hat{\boldsymbol{p}}_{i} \\
& =-i \frac{\hbar e}{m c} \sum_{i=1}^{N} e^{i \boldsymbol{k}_{h \nu} \cdot \boldsymbol{r}_{i}} \boldsymbol{\epsilon} \cdot \boldsymbol{\nabla}_{i}
\end{aligned}
$$

where $\boldsymbol{\epsilon}$ is the polarization vector (assumed to be constant in space) and $\boldsymbol{k}_{h \nu}$ is the momentum vector of the where $\hat{c}_{\boldsymbol{k}}$ annihilates an electron in Bloch state $\left|\Psi_{\kappa \boldsymbol{k}}\right\rangle$ with momentum $\boldsymbol{k}$ and band index $\kappa$ and $\hat{c}_{\boldsymbol{k}_{f}}^{\dagger}$ creates a photoelectron $\left|\Psi_{\kappa_{f} \boldsymbol{k}_{f}}\right\rangle$ with momentum $\boldsymbol{k}_{f}$ and band index $\kappa_{f}$. The matrix element $M_{\boldsymbol{k}_{f} \boldsymbol{k}}$ is defined as

$$
M_{\boldsymbol{k}_{f} \boldsymbol{k}}=-i \frac{\hbar e}{m c}\left\langle\Psi_{\kappa_{f} \boldsymbol{k}_{f}}\left|e^{i \boldsymbol{k}_{h \nu} \cdot \boldsymbol{r}} \boldsymbol{\epsilon} \cdot \nabla\right| \Psi_{\kappa \boldsymbol{k}}\right\rangle
$$

Within the "sudden approximation", we can assume the ionization process to be very rapid and the emitted photoelectron to be decoupled from the $N-1$ electrons remaining in the solid. The final state thus factorizes into the $(N-1)$ electron state $\left|\Psi_{f}^{N-1}\right\rangle$ with energy $E_{f}^{N-1}$ and the photoelectron state $\left|\Psi_{\kappa_{f} \boldsymbol{k}_{f}}\right\rangle$ with kinetic energy $E_{k i n}$ :

$$
\left|\Psi_{f}^{N}\right\rangle \sim \hat{\mathcal{A}}\left(\left|\Psi_{\kappa_{f} \boldsymbol{k}_{f}}\right\rangle \otimes\left|\Psi_{f}^{N-1}\right\rangle\right)=\hat{c}_{\boldsymbol{k}_{f}}^{\dagger}\left|\Psi_{f}^{N-1}\right\rangle
$$

The operator $\hat{\mathcal{A}}$ assures the proper anti-symmetrization of the associated $N$ electron wave functions. Eq. 1 can so be rewritten as

$$
\begin{aligned}
w_{f i} & =\frac{2 \pi}{\hbar}\left|\left\langle\Psi_{f}^{N-1}\left|\hat{c}_{\boldsymbol{k}_{f}} \sum_{\boldsymbol{k}_{f}^{\prime}, \boldsymbol{k}} M_{\boldsymbol{k}_{f}^{\prime} \boldsymbol{k}} \hat{c}_{\boldsymbol{k}_{f}^{\prime}}^{\dagger} \hat{c}_{\boldsymbol{k}}\right| \Psi_{i}^{N}\right\rangle\right|^{2} \delta\left(E_{f}^{N-1}+E_{k i n}-E_{i}^{N}-h \nu\right) \\
& =\frac{2 \pi}{\hbar}\left|\sum_{\boldsymbol{k}} M_{\boldsymbol{k}_{f} \boldsymbol{k}}\left\langle\Psi_{f}^{N-1}\left|\hat{c}_{\boldsymbol{k}}\right| \Psi_{i}^{N}\right\rangle\right|^{2} \delta\left(E_{f}^{N-1}+E_{k i n}-E_{i}^{N}-h \nu\right),
\end{aligned}
$$

where the sum $\sum_{\boldsymbol{k}}$ runs over the entire first Brillouin zone. Neglecting interference effects, i.e. assuming there is only one channel $\hat{c}_{\boldsymbol{k}}$ connecting the initial state $\left|\Psi_{i}^{N}\right\rangle$ with the (N-1) electron state $\left|\Psi_{f}^{N-1}\right\rangle$, we can approximate

$$
w_{f i}=\frac{2 \pi}{\hbar} \sum_{\boldsymbol{k}}\left|M_{\boldsymbol{k}_{f} \boldsymbol{k}}\right|^{2} \underbrace{\left|\left\langle\Psi_{f}^{N-1}\left|\hat{c}_{\boldsymbol{k}}\right| \Psi_{i}^{N}\right\rangle\right|^{2} \delta\left(E_{f}^{N-1}+E_{k i n}-E_{i}^{N}-h \nu\right)}_{\sum_{f} \Rightarrow A^{-}(\boldsymbol{k}, \omega)} .
$$

Summing the curly bracket over all possible $(N-1)$ electron eigenstates $\left|\Psi_{f}^{N-1}\right\rangle$, we identify the one-electron removal spectral function

$$
A^{-}(\boldsymbol{k}, \omega)=\sum_{f}\left|\left\langle\Psi_{f}^{N-1}\left|\hat{c}_{\boldsymbol{k}}\right| \Psi_{i}^{N}\right\rangle\right|^{2} \delta\left(\omega-E_{f}^{N-1}+E_{i}^{N}\right)
$$

which contains the many body effects of the system and 
is not further discussed in this work 1

The one-electron matrix element $M_{\boldsymbol{k}_{f} \boldsymbol{k}}$ depends largely on extrinsic factors like the photon momentum $\boldsymbol{k}_{h \nu}$, the polarization $\boldsymbol{\epsilon}$ and the photoelectron final state $\left|\Psi_{\kappa_{f} \boldsymbol{k}_{f}}\right\rangle$ and is the main focus of this tutorial.

\section{THE ONE-ELECTRON MATRIX ELEMENT}

While Siegbahn established electron spectroscopy for chemical analysis (ESCA) as a routine experimental technique (Gelius and Siegbahn, 1972, Nordling et al., 1957), first theoretical efforts to calculate the photoemission cross section quantitatively were on the way. Historically, the three-step model by Berglund and Spicer (1964) was one of the first approaches - dividing the photoemission process into three steps: photoelectron excitation; photoelectron transport to the surface; and photoelectron escape into the vacuum. Relativistic- and many body effects were completely neglected and both the initial- and the final states were treated - similarly as in this work - as stationary single-particle Bloch states of the infinite crystal. Later in the 1970s, authors such as Mahan (1970), Schaich and Ashcroft (1971), Gadzuk (1974a b. 1975) and Grobman (1978) studied the photoemission cross section of adsorbate systems, and developed sophisticated but somewhat complicated many body models. Feder and Meister (1969), Feder (1981), Feder et al. (1983), Liebsch (1974) and Liebsch and Plummer (1974), Liebsch (1976) and Spanjaard et al. (1977) further accounted for self-energy corrections in the final state, and for multiple scattering effects of the outgoing photoelectron in the semi-infinite crystal. This work was complemented by Pendry (1976) and Hopkinson and Pendry (1980) who implemented self energy corrections both for the initial and the final state in a one-step approach. Due to the photoelectron's spin as an additional source of information, relativistic effects were later taken into account by Borstel et al. (1981) and by Wöhlecke and Borstel (1983, 1984), and integrated into a relativistic onestep theory by Thörner and Borstel (1984) and Braun et al. (1985) in the 1980s, which has been continously developed further (for reviews see Braun (1996); Braun and Donath (2004); Ebert et al. (2011); Minár (2011, 2014); and Minár et al. (2011)).

Although modern many body one-step approaches are without doubt superior to simplistic one electron models, the numerical effort behind is cumbersome and the

\footnotetext{
${ }^{1}$ For an introductory discussion on the spectral function see for example Chuang (2001); Damascelli (2004); Damascelli et al. (2003); Hengsberger et al. (1999); Hüfner (1995] 2007); Lanzara et al. (2001); LaShell et al. (2000); Mahan (1993); Rotenberg (2010); Rotenberg and Kevan (2002); Shi et al. (2004); Valla et al. $(1999 \mathrm{a} \mathrm{b})$
}

results obtained can be beyond one's physical intuition. Thus, more simplistic but still powerful toy models were desired to capture the essence of the photoemission cross section from simple principles. In this spirit, authors such as Gobeli et al. (1964), Hermanson (1993), Anderson et al. (1978) and Borstel et al. (1979) developed selection rules for analyzing photoemission data based on symmetry arguments. Goldberg et al. (1981) calculated the orbital cross-sections of oriented atoms to explain the photoemission intensity variations obtained from solids, and Nemšák et al. (2012) incorporated these results into an interactive open access program (Fadley and Nemšák, 2014).

Based on such simple models, Ueno et al. (1997, 1993) determined the orientation of adsorbed molecules from ARPES intensity variations. More recently, the wave function properties of quantum well states Mugarza et al. 2000) and adsorbed organic molecules (Berkebile et al., 2008; Kera et al., 2006, Koller et al., 2007; Liu et al. 2014) were studied. And in some cases, even the real space electronic wave function could be reconstructed directly from the photoemission data (Jung et al., 2010, Lueftner et al., 2014, Mugarza et al., 2003, Puschnig et al. (2009). Moreover, simplistic models routinely serve to identify the orbital character of electronic band structure (Wang et al. , 2012) or even their spintexture (Zhu et al., 2013).

In this work, we will consistently develop one of the most simplistic approaches based on the three-step model: the free electron final state approximation. We assume all many body effects to be covered appropriately by the spectral contributions in Eq. 8 and outsource them into the spectral function of Eq. 9 . We further completely neglect multiple scattering or other lifetime effects and treat the outgoing photoelectron as nearly free. Surprisingly, we find in Sec. IV that the core of the photoemission intensity in a number of systems is described astonishingly well, which makes this model a useful tool for a rough estimation of photoemission intensity.

\section{A. Nearly free electron final state approximation}

In general, both the initial state $\left|\Psi_{\kappa \boldsymbol{k}}\right\rangle$ and the electron final state $\left|\Psi_{\kappa_{f} \boldsymbol{k}_{f}}\right\rangle$ have to respect the symmetry properties of the system. If the system under study is a gas of atoms, then both the initial and final state can be described by atomic wave functions with principal quantum number $n$, azimuthal quantum number $l$, and magnetic quantum number $m$, and the one-electron matrix element refers to an atomic transition $\left\langle n^{\prime} l^{\prime} m^{\prime}\left|e^{i \boldsymbol{k}_{h \nu} \cdot \boldsymbol{r}} \boldsymbol{\epsilon} \cdot \boldsymbol{\nabla}\right| n l m\right\rangle$, which in the long wavelength limit $e^{i \boldsymbol{k}_{h \nu} \cdot \boldsymbol{r}} \sim 1$ respects the well known dipole selection rules (Condon and Shortley, 1935, Cowan, 1981).

In an extended system, these atomic basis functions "combine" and form the wave function of a new eigen- 


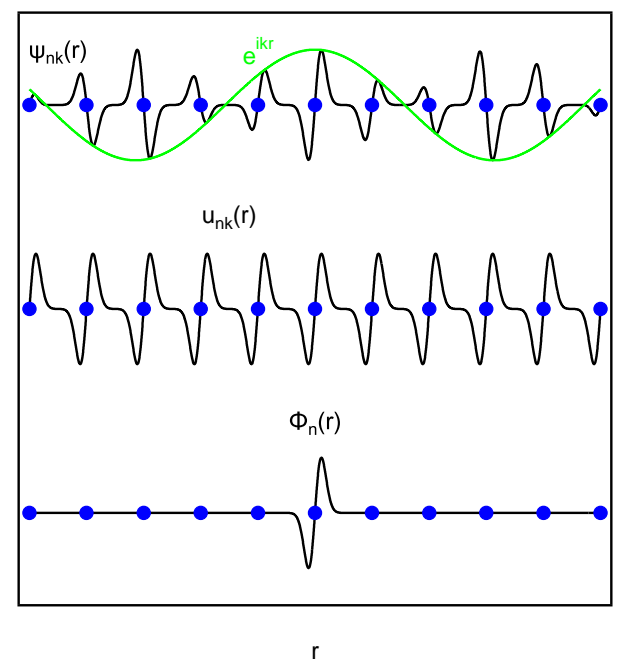

FIG. 1 Transformation from Bloch functions (top) to Wannier functions (bottom). (Top) Real-space representation of a Bloch function $\Psi_{\kappa k}(r)=\left\langle r \mid \Psi_{\kappa k}\right\rangle=e^{i k r} u_{k}(r)$ associated with a single band in 1D for one value of the wave vector $k$. Filled circles indicate lattice atoms, and the green line indicates the $e^{i k r}$ envelope. (Middle) Lattice periodic function $u_{k}(r)$ associated with the same band, forming periodic images of one another. (Bottom) Single site Wannier function $\Phi_{\kappa}(r)=\langle r \mid 0, \kappa\rangle$.

state. In the ordered crystalline solids we discuss here, translational symmetry requires both the initial and final state wave function to take the form of a Bloch wave. The new symmetry properties of this combined system then have an additional impact on the one-electron matrix element, and its associated selection rules.

As we will see later, it is convenient to develop the initial Bloch state $\left|\Psi_{\kappa \boldsymbol{k}}\right\rangle$ in terms of its localized Fourier components - the Wannier states

$$
|\boldsymbol{R}, \kappa\rangle=\frac{V}{(2 \pi)^{3}} \int_{B Z} d^{3} \boldsymbol{k} e^{-i \boldsymbol{k} \cdot \boldsymbol{R}}\left|\Psi_{\kappa \boldsymbol{k}}\right\rangle .
$$

$\boldsymbol{R}$ is a real space lattice vector, $V$ the real-space primitive cell volume and the integral is carried out over the entire Brillouin zone 2 The Bloch state so can be written as an inverse Fourier transform

$\left|\Psi_{\kappa \boldsymbol{k}}\right\rangle=\sum_{\boldsymbol{R}, R_{\perp}<0} e^{i \boldsymbol{k} \cdot \boldsymbol{R}}|\boldsymbol{R}, \kappa\rangle$,

where the sum runs over all lattice sites $\boldsymbol{R}$, which we artificially restrict to half space $R_{\perp}<0$. A simple example of how wave functions associated to Bloch- and Wannier states transform into each other is shown in Fig. 1.

If the kinetic energy of the photoelectron in the final state is sufficiently high, then it can be expected to behave independently/nearly free, i.e. to only weakly interact with the ions of the crystalline solid. It is thus convenient to develop $\left|\Psi_{\kappa_{f} \boldsymbol{k}_{f}}\right\rangle$ in terms of plane waves $e^{i \boldsymbol{k}_{f} \cdot \boldsymbol{r}} \equiv\left\langle\boldsymbol{r} \mid \boldsymbol{k}_{f}\right\rangle$ :

$$
\left|\Psi_{\kappa_{f} \boldsymbol{k}_{f}}\right\rangle=\sum_{\boldsymbol{G}} C_{\kappa_{f}}\left(\boldsymbol{k}_{f}-\boldsymbol{G}\right)\left|\boldsymbol{k}_{f}-\boldsymbol{G}\right\rangle
$$

where the sum is over reciprocal lattice vectors $\boldsymbol{G}$ (Henk, 2002, Henk et al. 1989, 1993). The coefficients $C_{\kappa_{f}}$ are solutions of the central equation (Kittel, 1996)

$$
\left(\frac{\hbar^{2} \boldsymbol{k}_{f}^{2}}{2 m}-E_{\boldsymbol{k}_{f}}^{\kappa_{f}}\right) C_{\kappa_{f}}\left(\boldsymbol{k}_{f}\right)+\sum_{\boldsymbol{G}} U_{\boldsymbol{G}} C_{\kappa_{f}}\left(\boldsymbol{k}_{f}-\boldsymbol{G}\right)=0,
$$

and $U_{G}$ are the Fourier coefficients of the crystal potential

$$
U(\boldsymbol{r})=\sum_{\boldsymbol{G}} U_{\boldsymbol{G}} e^{i \boldsymbol{G} \cdot \boldsymbol{r}}
$$

The matrix element thus can be written as

$$
\begin{aligned}
M_{\boldsymbol{k}_{f} \boldsymbol{k}} & \propto\left\langle\Psi_{\kappa_{f} \boldsymbol{k}_{f}}\left|e^{i \boldsymbol{k}_{h \nu} \cdot \boldsymbol{r}} \boldsymbol{\epsilon} \cdot \boldsymbol{\nabla}\right| \Psi_{\kappa \boldsymbol{k}}\right\rangle \\
& =\sum_{\boldsymbol{G}} C_{\kappa_{f}}^{*}\left(\boldsymbol{k}_{f}-\boldsymbol{G}\right) \sum_{\boldsymbol{R}, R_{\perp}<0} e^{i \boldsymbol{k} \cdot \boldsymbol{R}}\left\langle\boldsymbol{k}_{f}-\boldsymbol{G}-\boldsymbol{k}_{h \nu}|\boldsymbol{\epsilon} \cdot \boldsymbol{\nabla}| \boldsymbol{R}, \kappa\right\rangle \\
& =i \boldsymbol{\epsilon} \cdot \sum_{\boldsymbol{G}} C_{\kappa_{f}}^{*}\left(\boldsymbol{k}_{f}-\boldsymbol{G}\right) \sum_{\boldsymbol{R}, R_{\perp}<0} e^{i \boldsymbol{k} \cdot \boldsymbol{R}_{(}}\left(\boldsymbol{k}_{f}-\boldsymbol{G}-\boldsymbol{k}_{h \nu}\right)\left\langle\boldsymbol{k}_{f}-\boldsymbol{G}-\boldsymbol{k}_{h \nu} \mid \boldsymbol{R}, \kappa\right\rangle \\
& =i \boldsymbol{\epsilon} \cdot \sum_{\boldsymbol{G}} C_{\kappa_{f}}^{*}\left(\boldsymbol{k}_{f}-\boldsymbol{G}\right)\left(\boldsymbol{k}_{f}-\boldsymbol{G}\right) \sum_{\boldsymbol{R}, R_{\perp}<0} e^{-i\left(\boldsymbol{k}-\boldsymbol{k}_{f}+\boldsymbol{G}+\boldsymbol{k}_{h \nu}\right) \cdot \boldsymbol{R}} \underbrace{e^{i \boldsymbol{K}_{\|} \boldsymbol{R}_{\|}}}_{\equiv 1}\left\langle\boldsymbol{k}_{f}-\boldsymbol{G}-\boldsymbol{k}_{h \nu} \mid \mathbf{0}, \kappa\right\rangle,
\end{aligned}
$$

\footnotetext{
2 Note that the definition of the Wannier states implies an energy integration over the entire Bloch band, and the Wannier states are not Eigenstates of the Hamiltonian. For an introduction to
}

Wannier functions, see e.g. Brouder et al. (2007); Geller and Kohn (1993); Marzari et al. (2012); Panati and Pisante (2013); and Wannier (1937). 
where we made use of $\boldsymbol{\epsilon} \cdot \boldsymbol{k}_{h \nu}=0$, of $\boldsymbol{\nabla}^{\dagger}=-\nabla^{3}$ and where we performed a coordinate transformation $\boldsymbol{r} \rightarrow \boldsymbol{x}=\boldsymbol{r}-\boldsymbol{R}$. Further, the translation invariance $e^{i \boldsymbol{K}_{\|} \boldsymbol{R}_{\|}}=1$ with respect to some arbitrary in plane reciprocal lattice vector $\boldsymbol{K}_{\|}$ was taken into account. Writing $\sum_{\boldsymbol{R}, R_{\perp}<0}$ in terms of integrals parallel and perpendicular to the crystal surface $\frac{N}{V} \int d R_{\|} \int_{-\infty}^{0} d R_{\perp}$, the total matrix element becomes

$$
M_{\boldsymbol{k}_{f} \boldsymbol{k}} \propto 2 \pi i \frac{N}{V} \boldsymbol{\epsilon} \cdot \sum_{\boldsymbol{G}} C_{\kappa_{f}}^{*}\left(\boldsymbol{k}_{f}-\boldsymbol{G}\right)\left(\boldsymbol{k}_{f}-\boldsymbol{G}\right)\left\langle\boldsymbol{k}_{f}-\boldsymbol{G}-\boldsymbol{k}_{h \nu} \mid \mathbf{0}, \kappa\right\rangle \delta\left(\boldsymbol{k}_{\|}-\boldsymbol{k}_{f \|}+\boldsymbol{G}_{\|}+\boldsymbol{k}_{h \nu \|}+\boldsymbol{K}_{\|}\right) \frac{i}{k_{\perp}-k_{f \perp}+G_{\perp}+k_{h \nu \perp}} .
$$

\section{B. Free electron final state approximation}

As we saw in the previous section, both the the initialand the electron final state equally impact the oneelectron matrix element $M_{\boldsymbol{k}_{f} \boldsymbol{k}}$ and its associated selection rules. However, a proper description of the final state in a given system is typically difficult and not in reach for the experimentalist who tries to get a rough estimate on the photoemission intensity.

We thus imagine the most simple situation conceivable, and assume that the nearly free electron final state can be well approximated by one single plane wave component $\left|\boldsymbol{k}_{f}\right\rangle$. However, this does not accommodate the fact that photoemission is a surface process. For one, the penetration depth of the incoming photon beam is finite $(\mathcal{O}(100 \mathrm{~nm}))$. Second and important for our considerations, the outgoing electrons interact with the solid through electron-electron and electron-phonon interac- tion. Consequently, the electrons only possess a limited lifetime and are strongly damped with characteristic mean free paths of $\sim \mathcal{O}(5 \AA))^{4}$ A minimalistic model for the nearly free electron final state needs to take this damping into account, as e.g. described by an evanescent plane wave

$$
\left\langle\boldsymbol{r} \mid \boldsymbol{k}_{f}-\frac{i}{\lambda} \boldsymbol{e}_{\perp}\right\rangle=e^{i \boldsymbol{k}_{f} \boldsymbol{r}} e^{r_{\perp} / \lambda}
$$

where $\lambda$ is a phenomenological parameter describing the damping of the wave inside the solid (Fig. 2) (Henk, 2002 Henk et al. 1993). $\boldsymbol{e}_{\perp}$ is the surface normal vector. We thus have

$$
C_{\kappa_{f}}(\boldsymbol{k})=\delta\left(\boldsymbol{k}-\boldsymbol{k}_{f}+\frac{i}{\lambda} \boldsymbol{e}_{\perp}\right)
$$

$$
M_{\boldsymbol{k}_{f} \boldsymbol{k}} \propto 2 \pi i \frac{N}{V} \boldsymbol{\epsilon} \cdot \sum_{\boldsymbol{G}} \delta\left(\boldsymbol{G}+\frac{i}{\lambda} \boldsymbol{e}_{\perp}\right)\left(\boldsymbol{k}_{f}-\boldsymbol{G}\right)\left\langle\boldsymbol{k}_{f}-\boldsymbol{G}-\boldsymbol{k}_{h \nu} \mid \mathbf{0}, \kappa\right\rangle \delta\left(\boldsymbol{k}_{\|}-\boldsymbol{k}_{f \|}+\boldsymbol{G}_{\|}+\boldsymbol{k}_{h \nu \|}+\boldsymbol{K}_{\|}\right) \frac{i}{k_{\perp}-k_{f \perp}+G_{\perp}+k_{h \nu \perp}}
$$

which simplifies to

$$
M_{\boldsymbol{k}_{f} \boldsymbol{k}} \propto 2 \pi \frac{N}{V} \underbrace{\boldsymbol{\epsilon} \cdot\left(i \boldsymbol{k}_{f}-\frac{1}{\lambda} \boldsymbol{e}_{\perp}\right)}_{I} \underbrace{\left\langle\boldsymbol{k}_{f}-\boldsymbol{k}_{h \nu}+\frac{i}{\lambda} \boldsymbol{e}_{\perp} \mid \mathbf{0}, \kappa\right\rangle}_{I I} \underbrace{\delta\left(\boldsymbol{k}_{\|}-\boldsymbol{k}_{f \|}+\boldsymbol{k}_{h \nu \|}+\boldsymbol{K}_{\|}\right)}_{I I I} \underbrace{\frac{1}{i\left(k_{\perp}-k_{f \perp}+k_{h \nu \perp}\right)+1 / \lambda}}_{I V} .
$$

This expression is the central result of this work. We find four major contributions to the matrix element.

\footnotetext{
${ }^{3}$ We assume that $\Phi_{n}(\boldsymbol{r}-\boldsymbol{R}) \equiv\langle\boldsymbol{r} \mid \boldsymbol{R}, n\rangle$ is exponentially localized at lattice site $\boldsymbol{R}$ and thus $\lim _{r \rightarrow \infty} \Phi_{n}(\boldsymbol{r}-\boldsymbol{R})=0$. By partial integration we obtain $\int d^{3} \boldsymbol{r} \Psi^{*}(\boldsymbol{r}) \boldsymbol{\nabla} \phi_{n}(\boldsymbol{r}-\boldsymbol{R})=\left[\Psi^{*}(\boldsymbol{r}) \Phi_{n}(\boldsymbol{r}-\right.$ $\boldsymbol{R})]_{-\infty}^{\infty}-\int d^{3} \boldsymbol{r} \nabla \Psi^{*}(\boldsymbol{r}) \Phi_{n}(\boldsymbol{r}-\boldsymbol{R})=-\int d^{3} \boldsymbol{r} \nabla \Psi^{*}(\boldsymbol{r}) \Phi_{n}(\boldsymbol{r}-\boldsymbol{R})$ and thus $\nabla^{\dagger}=-\nabla$.

4 The electrons which lose energy on their way out of the solid are typically termed "secondary electrons". Their number as a function of binding energy is often times modeled by an integral "Shirley background" (Shirley 1972) or - more correct but also more complex - by a "Tougaard background" (Tougaard, 1990)
}

Term I contains the polarization information of the ARPES experiment. This term is zero whenever the photoelectrons on the way to the analyzer move perpendicular to the polarization vector. The analyzer slit of the photoelectron detector should thus be oriented to maximize the dot product $\boldsymbol{\varepsilon} \cdot \boldsymbol{k}_{f}$. Taking into account more but one plane wave component to the final state in Eq. $[16$ considerably weakens this criterion. This is particularly important at low photon energies as shown recently in graphene by Gierz et al. (2011). A detailed assessment of term I is given in Sec. III.E.

Term II describes the angular distribution of photoelectrons based on the local orbital properties. Es- 


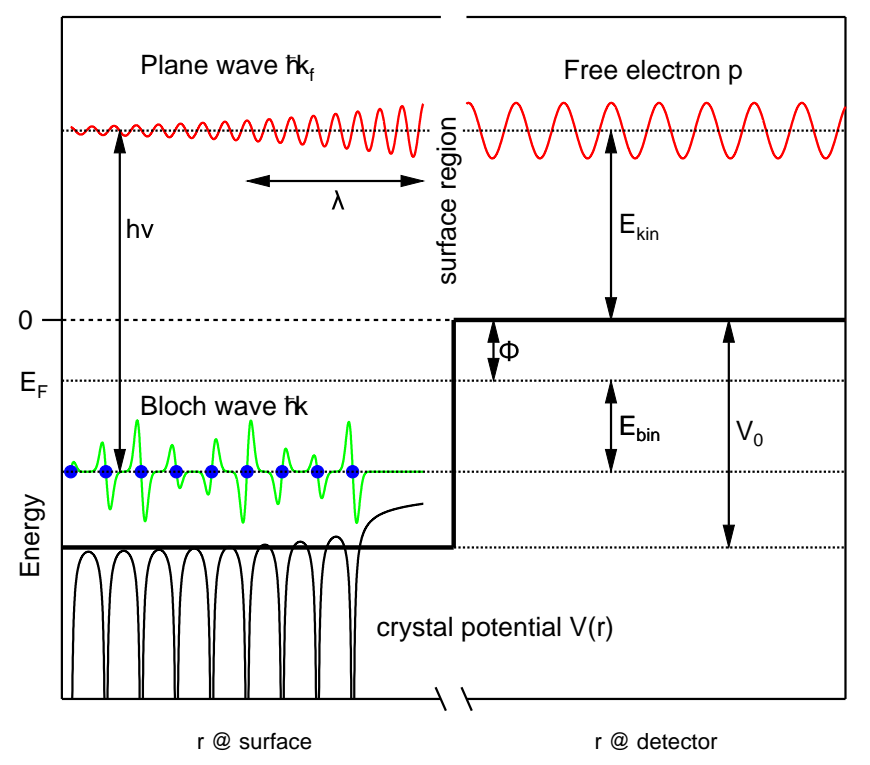

FIG. 2 Photoemission from an initial Bloch state $\hbar k$ into a damped final state plane wave $\hbar k_{f}$. Along the surface normal, translation symmetry is broken and the photoelectron is refracted.

sentially, it is given by the Fourier transform $\left\langle\boldsymbol{k}_{f}-\right.$ $\boldsymbol{k}_{h \nu}+\frac{i}{\lambda} \boldsymbol{e}_{\perp}|\mathbf{0}, \kappa\rangle$ of the damped Wannier orbital. Since the damping $1 / \lambda \sim \mathcal{O}\left(0.2 \AA^{-1}\right)$ and $k_{h \nu}=2 \pi \nu / c \sim$ $\mathcal{O}\left(0.05 \AA^{-1}\right)$ are typically small with respect to $k_{f} \sim$ $\sqrt{2 m E_{k i n}} \sim \mathcal{O}\left(5 \AA^{-1}\right)$ in UV- and soft x-ray photoemission with $h \nu \sim \mathcal{O}(100 \mathrm{eV}),\left\langle\boldsymbol{k}_{f}-\boldsymbol{k}_{h \nu}+\frac{i}{\lambda} \boldsymbol{e}_{\perp} \mid \mathbf{0}, \kappa\right\rangle$ will be typically well approximated by $\left\langle\boldsymbol{k}_{f} \mid \mathbf{0}, \kappa\right\rangle$. As the modulus square of a Fourier transform is equivalent to the Fourier transform of the autocorrelation function, the photoemission intensity can so be interpreted as a measure of the autocorrelation of the Wannier orbital $\langle\boldsymbol{r} \mid \mathbf{0}, \kappa\rangle$ associated to Bloch state $\left|\Psi_{\kappa \boldsymbol{k}}\right\rangle$. A more detailed discussion of term II is given in Sec. III.F.

Term III describes the in plane momentum conservation modulo some reciprocal lattice vector $\boldsymbol{K}_{\|}$due to the translational invariance of the lattice. In the UV and soft $\mathrm{x}$-ray regime, $k_{h \nu}$ can typically be neglected. Towards higher energies, i.e. towards the hard x-ray regime, deviations from the dipole approximation however become very important (Gray et al. 2012,2011 ; Papp et al. 2011 . Plucinski et al. 2008, Woicik, 2016). For the out of plane component IV, we find a Lorentzian "pseudo momentum conservation" $\left|M_{\boldsymbol{k}_{f} \boldsymbol{k}}\right|^{2} \propto \frac{1}{\left(k_{\perp}-k_{f \perp}+k_{h \nu \perp}\right)^{2}+1 / \lambda^{2}} \stackrel{\lambda \rightarrow \infty}{\longrightarrow}$ $\pi \delta\left(k_{\perp}-k_{f \perp}+k_{h \nu \perp}\right)$, which becomes more strict with larger penetration depth $\lambda$ of the final state. The sample surface thus can be viewed as a source of momentum to the photoelectron.

The introduction of the damping term $e^{r_{\perp} / \lambda}$ phenomenologically captures some important aspects of sur-

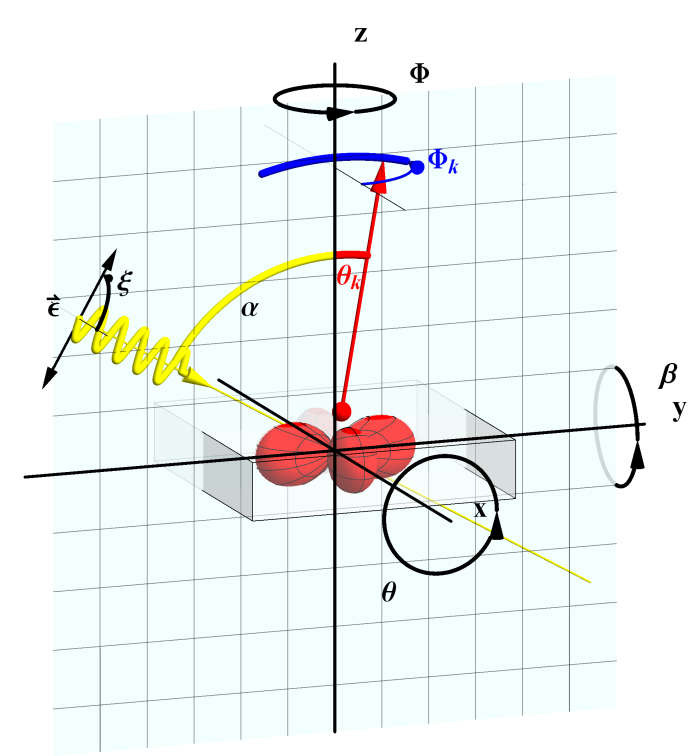

FIG. 3 Typical geometry of an ARPES setup with $\pi$ polarized light (yellow wiggles, $\xi=\pi / 2$ ) and $\pi$-polarized analyzer slits (blue curve, $\phi_{k}=\pi / 2$ ). All possible sample rotations are indicated by arrow-headed circles. The sample in this example can be rotated around $x$-, $y$ - and $z$-axis by dependent angles $\theta, \beta$ and $\phi$, respectively. At most synchrotrons, the polarization $\xi$ can be chosen as well. In a setup with a typical hemispherical analyzer, the orientation of the analyzer slit and thus $\phi_{k}$ of the photoelectron is typically fixed. whereas a whole angular range $\theta_{k}$ of photoelectrons (blue arc) enters the analyzer at the same time.

face photoemission. However, it has to be noted that this approach needs to be taken with great care, as lifetime effects can typically not be restricted to the final state alone. Moreover, the surface wave function is in general not very well approximated by a simple exponential cutoff of the bulk Bloch wave, but requires careful microscopic modeling. Even then, photoemission always measures the "convolution" of the initial and the final state close to the surface, where the details of the latter are largely neglected in this model.

\section{Photoelectron kinematics}

For completeness and consistency, we quickly derive the kinematic relations in between the photelectron momentum inside $\hbar \boldsymbol{k}_{f}$ and the photoelectron momentum $\boldsymbol{p}$ outside the solid (Damascelli, 2004, Eberhardt and Plummer, 1980, Hüfner, 1995, Plummer and Eberhardt, 1982). It is instructive to recall the propagation of an unbound free electron across a step potential as illustrated in Fig. 2: 


$$
V(\boldsymbol{r})=\left\{\begin{array}{cc}
-V_{0} & r_{\perp}<0 \\
0 & r_{\perp}>0
\end{array}\right.
$$

where $V_{0}$ is the inner potential of the solid. It describes the loss of kinetic energy of a photoelectron crossing the surface barrier and is roughly given by the sum of work function and valence band-width. Energy conservation during the emission process requires that $E\left(r_{\perp}<0\right)=$ $E\left(r_{\perp}>0\right)$ and thus

$$
\frac{\hbar^{2} \boldsymbol{k}_{f}^{2}}{2 m}=\frac{\boldsymbol{p}^{2}}{2 m}+V_{0}
$$

Since the in plane momentum $\hbar \boldsymbol{k}_{f \|}=\boldsymbol{p}_{\|}$remains conserved due to translation symmetry, the out of plane momentum has to absorb the inner potential and we find

$$
\frac{\hbar^{2} k_{f \perp}^{2}}{2 m}=\frac{p_{\perp}^{2}}{2 m}+V_{0} .
$$

In the typical ARPES geometry of Fig. 3, the emission angles $\left(\phi_{k}, \theta_{k}\right)$ and the kinetic energy $E_{k i n}$ of the photoelectron are measured, which relate to the photoelectron momentum as

$$
\boldsymbol{p}=\left(\begin{array}{c}
p_{x} \\
p_{y} \\
p_{z}
\end{array}\right)=\sqrt{2 m E_{k i n}} \times\left(\begin{array}{c}
\sin \theta_{k} \cos \phi_{k} \\
\sin \theta_{k} \sin \phi_{k} \\
\cos \theta_{k}
\end{array}\right)
$$

We thus have

$$
\begin{aligned}
\hbar^{2} \boldsymbol{k}_{f \|}^{2} & =\boldsymbol{p}_{\|}^{2}=p_{x}^{2}+p_{y}^{2}=2 m E_{k i n} \sin ^{2} \theta_{k} \\
\hbar^{2} k_{f \perp}^{2} & =p_{\perp}^{2}+2 m V_{0}=2 m\left(E_{k i n} \cos ^{2} \theta_{k}+V_{0}\right),
\end{aligned}
$$

and finally

$$
\begin{aligned}
& k_{f \|}=\frac{1}{\hbar} \sqrt{2 m E_{k i n}} \sin \theta_{k} \\
& k_{f \perp}=\frac{1}{\hbar} \sqrt{2 m\left(E_{k i n} \cos ^{2} \theta_{k}+V_{0}\right)} .
\end{aligned}
$$

According to Fig. 2, the kinetic energy $E_{k i n}$ further is related to the binding energy $E_{B}$ of the electron via photon energy $h \nu$ and the work function $\Phi$ of the solid: $E_{k i n}=h \nu-E_{B}-\Phi 5$

\footnotetext{
5 Note that in the experiment, the photoelectron has to be "absorbed" by the electron analyzer. The analyzer is in electrical equilibrium with the sample and thus the quantity $\Phi$ which enters here is actually the work function of the photoelectron analyzer.
}

\section{The electron escape depth $\lambda$}

In the last sections, we introduced $\lambda$ as a phenomenological parameter to describe the finite penetration depth of the final state, i.e. the information- or probing- depth of the photoemission experiment. Even though the validity of one single $\lambda$-parameter to describe the surface is certainly debatable, we may attribute it to the inelastic mean free path of the photoelectron. $\lambda$ then corresponds to the electron's average travel distance in between successive inelastic collisions, which can be calculated quite accurately from models (Tanuma et al., 1987, 1988) which are continuously improved further (Shinotsuka et al. 2015). Additional elastic scattering might play a role as well and $\lambda$ could be interpreted in terms of the effective attenuation length, which can be determined from over layer experiments (Graber et al. 2011).

Mostly however, $\lambda$ is determined by electron-electron interaction. In the energy range of $E_{k i n} \sim \mathcal{O}(100 \mathrm{eV})$, the nearly free electron final state approximation applies and the electron-electron interaction is determined by the plasma frequency of the solid. This quantity only depends on the free electron density and is largely independent of the constituents of the solid. Hence, $\lambda$ is probably fairly well described by the empirical "universal curve" developed by Seah and Dench (1979)

$$
\lambda=c_{1} E^{-2}+c_{2} \sqrt{E}
$$

where $E=E_{k i n}+\Phi$ is the electrons' energy above the Fermi level and $c_{1}$ and $c_{2}$ are material parameters. In the $50-100 \mathrm{eV}$ energy range, $\lambda$ is as low as a few $\AA$. This underlines the high surface sensitivity of photoemission, which requires atomically clean surfaces prepared under ultra high vacuum conditions.

\section{E. The polarization term I: $\boldsymbol{\epsilon} \cdot\left(i \boldsymbol{k}_{f}-\frac{1}{\lambda} \boldsymbol{e}_{\perp}\right)$}

The influence of the experimental geometry on the distribution of spectral weight relies primarily on the projection of the polarization vector onto the final state wave vector $\boldsymbol{\epsilon} \cdot\left(i \boldsymbol{k}_{f}-\frac{1}{\lambda} \boldsymbol{e}_{\perp}\right)$. In the following we will see that this term has a major impact on the distribution of photoelectrons which is important to be taken into account in the design of experimental setups. Besides, this term plays a major role for the calibration and interpretation of dichroism data.

\section{Elliptically polarized light}

We start from a general ARPES configuration as depicted in Fig. 3. The polarization vector $\epsilon$ can be written in Jones notation 


$$
\boldsymbol{\epsilon}=\epsilon e^{i \eta}\left(\begin{array}{c}
\cos \xi \\
e^{i \delta} \cos \alpha \sin \xi \\
e^{i \delta} \sin \alpha \sin \xi
\end{array}\right)
$$

where $\alpha$ is the angle in between incoming x-ray beam and electron analyzer, $\xi$ defines the ratio and $\delta$ defines the relative phase in between the principal components of $\epsilon$, respectively. If $\delta=0$, then $\xi$ is identical to the angle in between $\boldsymbol{\epsilon}$ and the $x$-axis. $\eta$ is the absolute phase of the light which cancels in the calculation of the modulus square of the matrix element and is just noted for completeness.

To assess the polarization part of the matrix element, it is useful to rewrite its modulus square $\left|\boldsymbol{\epsilon} \cdot\left(i \boldsymbol{k}_{f}-\frac{1}{\lambda} \boldsymbol{e}_{\perp}\right)\right|^{2}$ in terms of Stokes parameters

$$
\begin{aligned}
& S_{0}=\epsilon^{2} \\
& S_{1}=\epsilon^{2} \cos (2 \xi) \\
& S_{2}=\epsilon^{2} \cos \delta \sin (2 \xi) \\
& S_{3}=\epsilon^{2} \sin \delta \sin (2 \xi),
\end{aligned}
$$

which are typically used to characterize the degree of polarization (Bahrdt et al. 2010; Finetti et al., 2004, Koide et al. 1993, 1991; Nahon and Alcaraz, 2004 Nahon et al., 2012 , Saito et al., 2014, Uschakow et al., 2013; Yamamoto et al. 2014). Using Eq. 28 and after some algebra, we find

$$
\begin{aligned}
\left|\boldsymbol{\epsilon} \cdot\left(i \boldsymbol{k}_{f}-\frac{1}{\lambda} \boldsymbol{e}_{\perp}\right)\right|^{2} & =\frac{S_{0}}{2}\left(k_{f x}^{2}+k_{f y}^{\prime 2}+\frac{\sin ^{2} \alpha}{\lambda^{2}}\right) \\
& +\frac{S_{1}}{2}\left(k_{f x}^{2}-k_{f y}^{\prime 2}-\frac{\sin ^{2} \alpha}{\lambda^{2}}\right) \\
& +S_{2} k_{f x} k_{f y}^{\prime}-S_{3} k_{f x} \frac{\sin \alpha}{\lambda},
\end{aligned}
$$

where $k_{f y}^{\prime}=\cos \alpha k_{f y}+\sin \alpha k_{f z}$.

We note that each Stokes parameter introduces a contribution of different symmetry to the distribution of photoelectrons. A particularly interesting example is circular dichroism. In the case of perfectly circular polarized

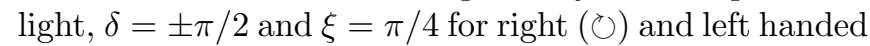
$(\circlearrowleft)$ polarization. We consequently encounter a nonzero contribution to the circular dichroism intensity

$$
\left|M_{\boldsymbol{k}_{f} \boldsymbol{k}}^{\circlearrowright}\right|^{2}-\left|M_{\boldsymbol{k}_{f} \boldsymbol{k}}^{\circlearrowleft}\right|^{2} \propto-\frac{2 \epsilon^{2} k_{f x} \sin \alpha}{\lambda}\left|\left\langle\boldsymbol{k}_{f} \mid \mathbf{0}, \kappa\right\rangle\right|^{2},
$$

which reflects the handedness of the photoemission experiment $\left(\alpha \neq 0\right.$ and $k_{f x} \neq 0$ for a finite $\left.\lambda\right)$. According to Eq. 25, the circular dichroism asymmetry

$$
\frac{\left|M_{\boldsymbol{k}_{f} \boldsymbol{k}}^{\circlearrowright}\right|^{2}-\left|M_{\boldsymbol{k}_{f} \boldsymbol{k}}^{\circlearrowleft}\right|^{2}}{\left|M_{\boldsymbol{k}_{f} \boldsymbol{k}}^{\circlearrowright}\right|^{2}+\left|M_{\boldsymbol{k}_{f} \boldsymbol{k}}^{\circlearrowleft}\right|^{2}} \propto \frac{2 k_{f x} \sin \alpha / \lambda}{k_{f x}^{2}+k_{f y}^{\prime 2}+\frac{\sin ^{2} \alpha}{\lambda^{2}}}
$$

gives a complex expression in terms of kinetic energy $E_{k i n}$ and emission angles $\phi_{k}$ and $\theta_{k}$ :

$$
\frac{\left|M_{\boldsymbol{k}_{f} \boldsymbol{k}}^{\circlearrowright}\right|^{2}-\left|M_{\boldsymbol{k}_{f} \boldsymbol{k}}^{\circlearrowleft}\right|^{2}}{\left|M_{\boldsymbol{k}_{f} \boldsymbol{k}}^{\circlearrowright}\right|^{2}+\left|M_{\boldsymbol{k}_{f} \boldsymbol{k}}^{\circlearrowleft}\right|^{2}} \propto \frac{2 \sqrt{x} \sin \alpha \cos \phi_{k} \sin \theta_{k}}{\sin ^{2} \alpha+x \cos ^{2} \phi_{k} \sin ^{2} \theta_{k}+\left(\sqrt{x \cos ^{2} \theta_{k}+y} \sin \alpha+\sqrt{x} \cos \alpha \sin \phi_{k} \sin \theta_{k}\right)^{2}}
$$

where we conveniently define the dimensionless quantities $x=\frac{2 m}{\hbar^{2}} \lambda^{2} E_{k i n}$ and $y=\frac{2 m}{\hbar^{2}} \lambda^{2} V_{0}$. For $\phi_{k}=0$ and $\theta_{k}=$ $\pi / 2$ this expression simplifies to

$$
\frac{\left|M_{\boldsymbol{k}_{f} \boldsymbol{k}}^{\circlearrowright}\right|^{2}-\left|M_{\boldsymbol{k}_{f} \boldsymbol{k}}^{\circlearrowleft}\right|^{2}}{\left|M_{\boldsymbol{k}_{f} \boldsymbol{k}}^{\circlearrowright}\right|^{2}+\left|M_{\boldsymbol{k}_{f} \boldsymbol{k}}^{\circlearrowleft}\right|^{2}} \propto \frac{2 \sqrt{x} \sin \alpha}{(y+1) \sin ^{2} \alpha+x} .
$$

For $\alpha \rightarrow \pi / 2$, we obtain an upper estimate of the geometric dichroism signal to expect. We find a maximum value of $1 / \sqrt{1+y}$ at $x_{0}=(y+1) \sin ^{2} \alpha$. Interestingly, both magnitude and position of the maximum depend sensitively on the inner potential $V_{0}$ and the penetration depth $\lambda$. Fig. 4 shows a plot of the circular dichroism asymmetry as function of the parameter $\lambda^{2} E_{k i n}$. Clearly, the geometric dichroism signal is maximum in the UV region and is softened by both an increasing $\lambda$ and an increasing inner potential $V_{0}$. Fig. 5 is a similar plot, where we assume that $\lambda\left(E_{k i n}\right)$ is a function of the kinetic energy phenomenologically described by the universal curve of Eq. 27 (Seah and Dench, 1979). In the UV energy range, geometric dichroism can be as high as $5-10 \%$, but is expected to fade away for high kinetic energies.

The experimental handedness causing nonzero dichroism is introduced by the incoming light vector $\boldsymbol{k}_{h \nu}$, outgoing photoelectron vector $\boldsymbol{k}_{f}$ and the surface normal vector $\frac{1}{\lambda} \boldsymbol{e}_{\perp}$ (Jahnke et al., 2002, Westphal et al., 1989). In a similar fashion, such an experimental handedness can be introduced by the chirality of the system itself (Schönhense, 1990), or by magnetism (Fanelsa et al. 1996).

Even if the role of this handedness (here the attenuation length $\lambda$ ) can be neglected, we note that the degree of polarization 


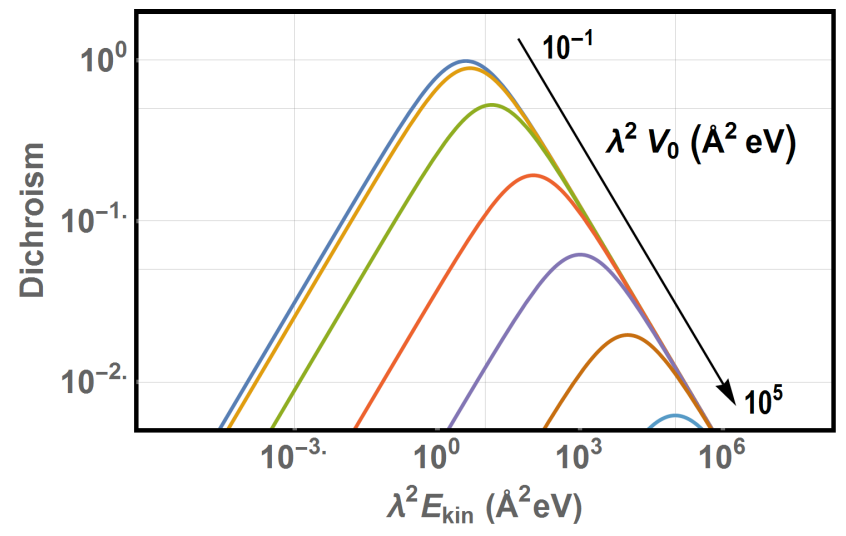

FIG. 4 Geometry induced circular dichroism asymmetry as a function of $\lambda^{2} E_{k i n}$ and calculated with $\log \left(\lambda^{2} V_{0}\right)=[-1 ; 5]$, $\alpha=\pi / 2, \theta_{k}=\pi / 2$ and $\phi_{k}=0$.

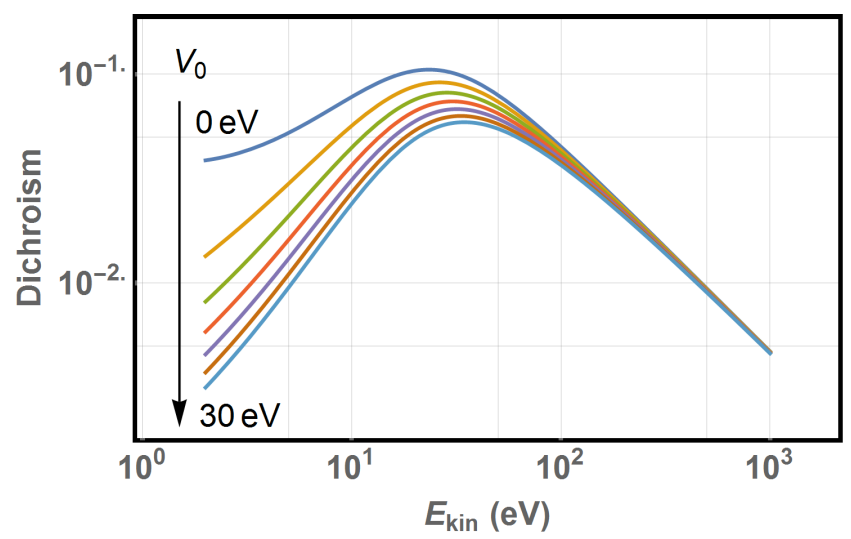

FIG. 5 Geometry induced circular dichroism asymmetry calculated for $\lambda$ according to the universal curve of Eq. 27] with $c_{1}=538$ and $c_{2}=0.41$ (Seah and Dench, 1979); $\alpha=\pi / 3$, $\theta_{k}=\pi / 2, \phi_{k}=0$ and $V_{0}=[0 ; 30]$.

$$
\Pi=\frac{\sqrt{S_{1}^{2}+S_{2}^{2}+S_{3}^{2}}}{S_{0}}
$$

in most experiments is typically lower than $100 \%$ and largely depends on the characteristics of the light source (e.g. the state of the undulator and its downstream optical components). This implies that the Stokes parameters for a given polarization state typically deviate from their nominal value, which according to Eq. 30 can result in geometric dichroism of quite complex symmetry. We further note that the degree of polarization at a beamline typically varies as a function of the photon energy (i.e. as a function of the undulator gap and phase), and the geometry derived dichroism asymmetry can therefore vary and potentially even change sign.

In view of these arguments, a proper calibration and characterization of the polarization state is of primordial importance to reliably separate these extrinsic from the interesting intrinsic dichroism effects of various origins which are not captured by our simplistic formalism Arpiainen et al. 2008; Ärrälä et al., 2013, Bian et al., 2012, Chassé and Rennert, 1997; Daimon et al., 1993, Fecher et al. 2002; Liu et al., 2011; Park and Louie, 2012, Park et al. 2012; Sánchez-Barriga et al., 2014, Schönhense, 1990: Wang et al., 2011, Zhu et al., 2013).

\section{Linearly polarized light}

In a linearly polarized configuration, $\delta=0$ and the polarization vector $\epsilon$ reduces to

$$
\boldsymbol{\epsilon}=\epsilon e^{i \eta}\left(\begin{array}{c}
\cos \xi \\
\cos \alpha \sin \xi \\
\sin \alpha \sin \xi
\end{array}\right),
$$

where $\xi$ is the orientation of the polarization vector as defined in Fig. 3. For $\sigma$-polarized light, $\xi=0$ and the polarization vector is perpendicular to the scattering plane. Only the Stokes parameters $S_{1}=S_{0}$ are nonzero and the polarization dependent term I becomes $\left|\boldsymbol{\epsilon} \cdot\left(i \boldsymbol{k}_{f}-\frac{1}{\lambda} \boldsymbol{e}_{\perp}\right)\right|^{2}=S_{0} k_{f x}^{2}$, which vanishes for $k_{f x}=0$. We note however that this suppression is considerably weakened once several plane wave components are taken into account in the final state (Gierz et al., 2011).

For $\pi$-polarized light, $\xi=\pi / 2$ and the polarization vector is in the scattering plane. Now $S_{1}=-S_{0}$ and thus $\left|\boldsymbol{\epsilon} \cdot\left(i \boldsymbol{k}_{f}-i \frac{1}{\lambda} \boldsymbol{e}_{\perp}\right)\right|^{2}=S_{0}\left(k_{f y}^{\prime 2}+\frac{\sin ^{2} \alpha}{\lambda^{2}}\right)$. Assuming $\lambda \rightarrow \infty$, this quantity vanishes for $k_{f y}=-k_{f z} \tan \alpha$. Examples for the effect of linear polarization on the distribution of photoelectrons emitted from $s$-, $p$ - and $d$-orbitals are shown in Fig. 6 .

\section{Unpolarized light}

Unpolarized light refers to the situation of light with polarization vectors distributed isotropically within a plane perpendicular to the incoming light direction but without a component parallel to it, e.g. provided by a Helium lamp. In this geometry, we have to integrate the polarization term over $\xi$ and obtain

$$
\left|M_{\boldsymbol{k}_{f} \boldsymbol{k}}\right|^{2} \propto \int_{0}^{\pi}\left|\boldsymbol{\epsilon} \cdot\left(i \boldsymbol{k}_{f}-\frac{1}{\lambda} \boldsymbol{e}_{\perp}\right)\right|^{2} \sin \xi d \xi=\frac{2}{3} \epsilon^{2}\left(k_{f}^{2}+\frac{1}{\lambda^{2}}+2 k_{f y} k_{f z} \sin (2 \alpha)+\left(k_{f y}^{2}-k_{f z}^{2}-\frac{1}{\lambda^{2}}\right) \cos (2 \alpha)\right)
$$


The polarization derived distribution of photoemission intensity expected from an unpolarized light source is clearly not isotropic, but can introduce nontrivial symmetries.

\section{Isotropic light}

Here, we refer to the hypothetical situation of light with polarization vectors distributed isotropically along all directions of 3D space as isotropic light. In this configuration, we can additionally integrate over $\alpha$ and obtain

$$
\begin{aligned}
\left|M_{\boldsymbol{k}_{f} \boldsymbol{k}}\right|^{2} & \propto \int_{0}^{2 \pi} \int_{0}^{\pi}\left|\boldsymbol{\epsilon} \cdot\left(i \boldsymbol{k}_{f}-\frac{1}{\lambda} \boldsymbol{e}_{\perp}\right)\right|^{2} \sin \xi d \xi d \alpha \\
& =\frac{4}{3} \pi \epsilon^{2}\left(k_{f}^{2}+\frac{1}{\lambda^{2}}\right) .
\end{aligned}
$$

As expected, the intensity distribution is now fully isotropic.

\section{F. The orbital term II: $\left\langle\boldsymbol{k}_{f}-\boldsymbol{k}_{h \nu}+\frac{i}{\lambda} \boldsymbol{e}_{\perp} \mid \mathbf{0}, \kappa\right\rangle$}

As we saw, the total matrix element $M_{\boldsymbol{k}_{f} \boldsymbol{k}}$ is to a large extent determined by the Fourier Transform $\left\langle\boldsymbol{k}_{f}-\right.$ $\boldsymbol{k}_{h \nu}+\frac{i}{\lambda} \boldsymbol{e}_{\perp}|\mathbf{0}, \kappa\rangle$ of the damped localized Wannier function $\langle\boldsymbol{r} \mid \mathbf{0}, \kappa\rangle$ associated to the Bloch state $\left|\Psi_{\kappa \boldsymbol{k}}\right\rangle$. In complex systems, the determination of the Wannier function can be a tedious task and is typically subject to the field of density functional theory. In many cases however, the Wannier state can be approximated by a tight binding form, i.e. a linear combination

$$
|\mathbf{0}, \kappa\rangle \sim \sum_{\boldsymbol{R}_{\boldsymbol{j}}} \sum_{n l m} c_{j n l m}^{\kappa}(\boldsymbol{k})\left|\boldsymbol{R}_{j}, n l m\right\rangle
$$

of atomic orbitals $\left|\boldsymbol{R}_{j}, n l m\right\rangle$ with quantum numbers $n$, $l$, and $m$, centered at coordinates $\boldsymbol{R}_{j}$ within the unit cell (Goringe et al., 1997; Koster and Slater, 1954). The coefficients $c_{j n l m}^{\kappa}(\boldsymbol{k})$ are the solutions of the tight-binding Hamiltonian, which in general depend on $\boldsymbol{k}$.

The Bloch state $\left|\Psi_{\kappa \boldsymbol{k}}\right\rangle$ has now the same form as the Bloch-sum formula in tight-binding theory

$$
\left|\Psi_{\kappa \boldsymbol{k}}\right\rangle=\sum_{\boldsymbol{R}} e^{i \boldsymbol{k} \cdot \boldsymbol{R}} \sum_{\boldsymbol{R}_{j}} \sum_{n l m} c_{j n l m}^{\kappa}(\boldsymbol{k})\left|\boldsymbol{R}+\boldsymbol{R}_{j}, n l m\right\rangle,
$$

where the Wannier state is replaced by the tight binding form of Eq. 39 and $\boldsymbol{R}$ labels all lattice sites of the solid.

Term II of the total matrix element can now be written as

$$
\begin{aligned}
M_{\boldsymbol{k}_{f} \boldsymbol{k}}^{\kappa} & \propto\left\langle\boldsymbol{k}_{f}-\boldsymbol{k}_{h \nu}+\frac{i}{\lambda} \boldsymbol{e}_{\perp}\left|\sum_{\boldsymbol{R}_{j}} \sum_{n l m} c_{j n l m}^{\kappa}(\boldsymbol{k})\right| \boldsymbol{R}_{j}, n l m\right\rangle \\
& =\sum_{\boldsymbol{R}_{j}} \sum_{n l m} c_{j n l m}^{\kappa}(\boldsymbol{k})\left\langle\boldsymbol{k}_{f}-\boldsymbol{k}_{h \nu}+\frac{i}{\lambda} \boldsymbol{e}_{\perp} \mid \boldsymbol{R}_{j}, n l m\right\rangle \\
& =\sum_{\boldsymbol{R}_{j}} \sum_{n l m} c_{j n l m}^{\kappa}(\boldsymbol{k}) e^{-i\left(\boldsymbol{k}_{f}-\boldsymbol{k}_{h \nu}+\frac{i}{\lambda} \boldsymbol{e}_{\perp}\right) \cdot \boldsymbol{R}_{j}}\left\langle\boldsymbol{k}_{f}-\boldsymbol{k}_{h \nu}+\frac{i}{\lambda} \boldsymbol{e}_{\perp} \mid \mathbf{0}, n l m\right\rangle \\
& =\sum_{\boldsymbol{R}_{j}} \sum_{n l m} c_{j n l m}^{\kappa}(\boldsymbol{k}) e^{-i\left(\boldsymbol{k}_{f}-\boldsymbol{k}_{h \nu}+\frac{i}{\lambda} \boldsymbol{e}_{\perp}\right) \cdot \boldsymbol{R}_{j}} M_{\boldsymbol{k}_{f}}^{n l m} \\
& \sim \sum_{\boldsymbol{R}_{j}} \sum_{n l m} c_{j n l m}^{\kappa}\left(\boldsymbol{k}_{f}-\boldsymbol{k}_{h \nu}-\boldsymbol{K}_{\|}\right) e^{-i\left(\boldsymbol{k}_{f}-\boldsymbol{k}_{h \nu}+\frac{i}{\lambda} \boldsymbol{e}_{\perp}\right) \cdot \boldsymbol{R}_{j}} M_{\boldsymbol{k}_{f}}^{n l m}
\end{aligned}
$$

where we exploited the quasi momentum conservation in the last step and wrote

$$
M_{\boldsymbol{k}_{f}}^{n l m} \propto\langle\boldsymbol{k}_{f}-\boldsymbol{k}_{h \nu}+\frac{i}{\lambda} \boldsymbol{e}_{\perp} \underbrace{|\mathbf{0 , n l m}\rangle}_{\equiv|n l m\rangle} .
$$

In the same fashion as the atomic wave functions combine and form the wave function of the Bloch state, the atomic matrix elements combine and form a generalized matrix element, reflecting the translational invariance of the solid as well as the local symmetry of the orbital. The main task is hence to perform a weighted sum over all Fourier components $M_{\boldsymbol{k}_{f}}^{n l m}$ of the damped atomic orbitals in the unit cell, which gives expression Eq. 41 the shape of a "form factor".

\section{The angular cross-section}

If the penetration depth of the final state can be neglected $(\lambda \rightarrow \infty)$, then we are left with calculating the 


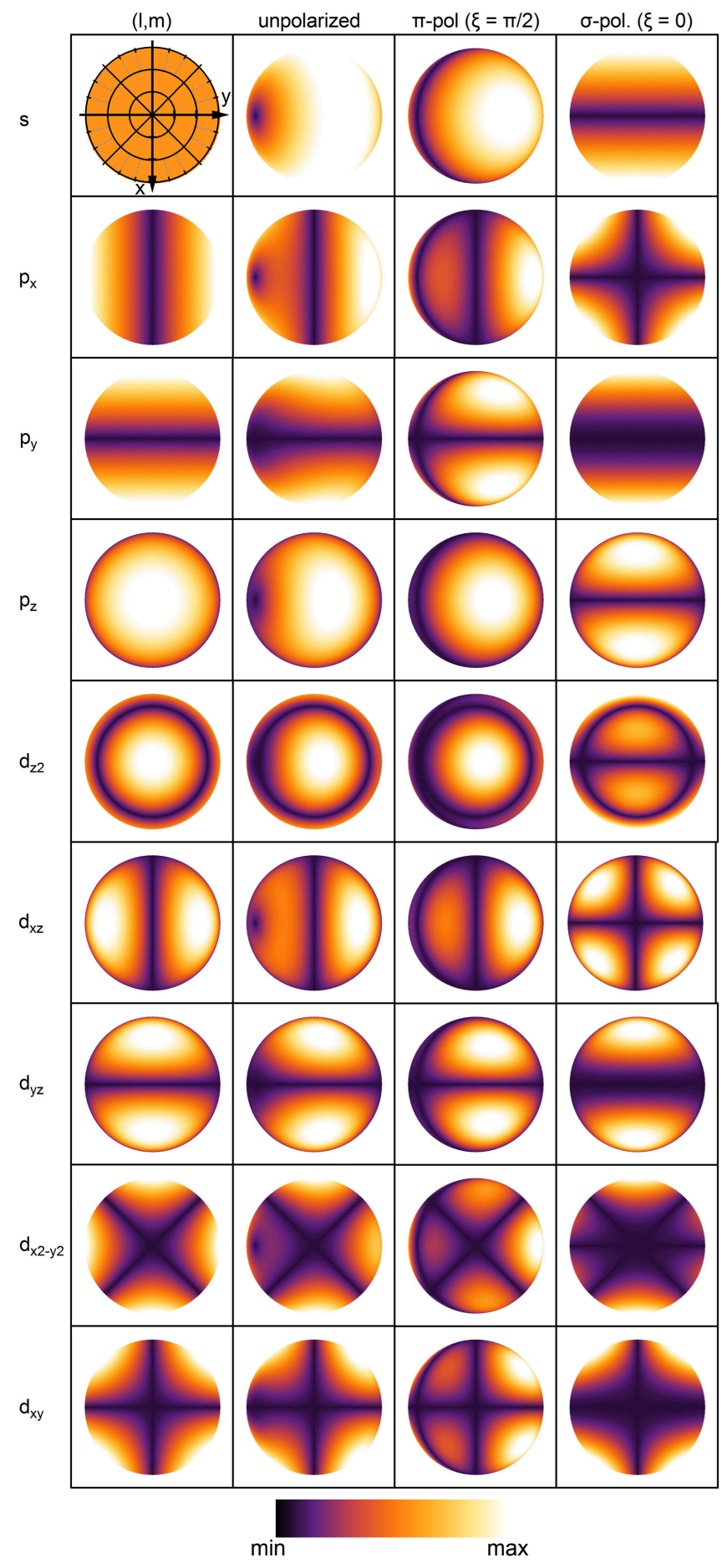

FIG. 6 Stereographic projection of the angular distribution $\left|M_{\boldsymbol{k}_{f}}^{n l m}\right|$ of photoelectrons emitted from $s$-, $p$ - and $d$-orbitals for different polarizations $\xi$ and an incident light angle of $\alpha=60^{\circ}$ (light coming from left). Whereas the photoelectron distribution in the unpolarized- and $\pi$-polarized light configurations essentially reflect symmetry of the atomic orbital $(l, m), \sigma$ polarization causes significant suppression of spectral weight along the $y z$ scattering plane.
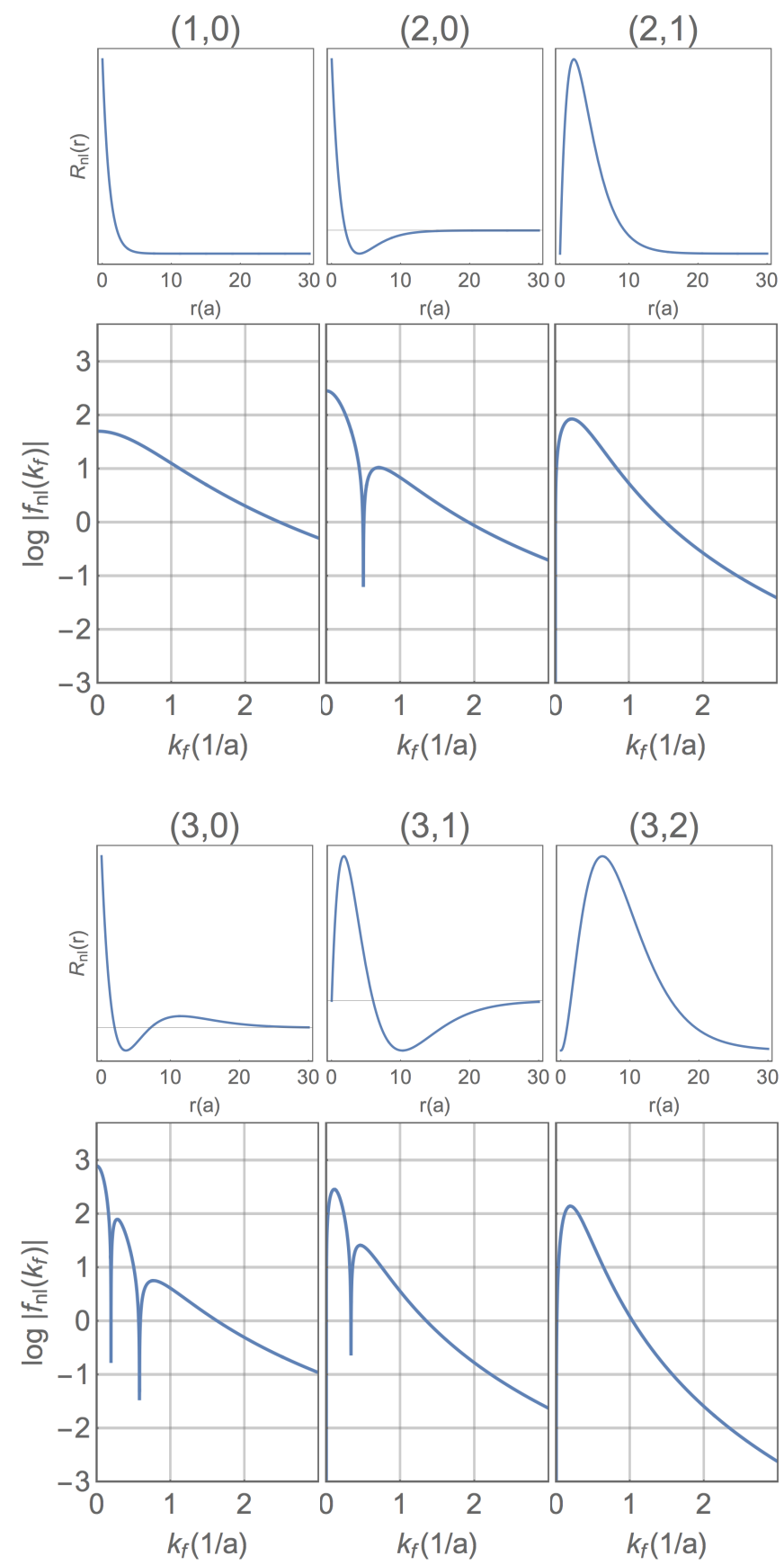

FIG. 7 The radial contribution to the matrix element $\left|f_{n l}\left(k_{f}\right)\right|$ calculated for several radial atomic wave functions $R_{n l}(r)$. At distinct values of $k_{f}$, sharp "Cooper minima" are observed.

Fourier transform of atomic wave functions of the form

$$
\Phi(\boldsymbol{r})=\langle\boldsymbol{r} \mid n l m\rangle=R_{n l}(r)\langle\boldsymbol{r} \mid l m\rangle,
$$

where $\boldsymbol{r}=\left\{r, \theta_{r}, \phi_{r}\right\}, R_{n l}(r)$ is the radial wave function, and $\langle\boldsymbol{r} \mid l m\rangle$ is a sherical harmonics. Podolsky and Pauling (1929) have shown that functions of this form possess a Fourier transform 


$$
\tilde{\Phi}\left(\boldsymbol{k}_{f}\right)=f_{n l}\left(k_{f}\right)\left\langle\boldsymbol{k}_{f} \mid l m\right\rangle,
$$

where $\boldsymbol{k}_{f}=\left\{k_{f}, \theta_{k}, \phi_{k}\right\}$, and the angular part remains invariant (Arfken et al. 2012, Drake and Cassar, 2006). The angular distribution of photoelectrons associated to a certain atomic orbital therefore reflects the symmetry of the orbital itself.

Exemplary plots of the angular distribution of $s, p$ and $d$ orbitals are shown in the left column " $(\mathrm{lm})$ " of Fig. 6 . These are identical to the angular distribution of photoelectrons emitted from these orbitals in a hypothetical isotropic light configuration (cf. Sec. III.E.4). Taking into account the polarization term I for unpolarized light (second column, cf. Sec. III.E.3, e.g. obtained from a helium lamp), the photoelectron distribution deviates slightly but overall still captures the orbital distribution very well. The third column shows the result for a $\pi$ polarized light configuration (cf. III.E.2). The polarization vector now suppresses all spectral components in the plane perpendicular to the incoming light polarization. If the angle between incoming light and detector $\alpha$ is sufficiently large, this leaves the photoelectrons close to normal emission basically unaffected and the angular distribution of photoelectrons still reflects the orbital symmetry. In $\sigma$-polarization, this node is always along the $y z$ scattering plane and the photoelectron distribution deviates significantly from the underlying orbital distribution. It is noted however that the suppression of spectral weight due to the polarization term is much less severe in the actual experiment as it is significantly weakened by additional plane wave contributions to the final state. Nevertheless a reduction of the photoemission cross section in $\sigma$-with respect to $\pi$-polarization can be observed quite generally.

\section{The radial cross-section}

Whereas the Fourier transform of the spherical harmonics $\langle\boldsymbol{r} \mid l m\rangle$ is just the same spherical harmonics $\left\langle\boldsymbol{k}_{f} \mid l m\right\rangle$ in Fourier space, the radial part $R_{n l}(r)$ gives a Hankel transform

$$
f_{n l}\left(k_{f}\right)=4 \pi(-i)^{l} \int_{0}^{\infty} d r r^{2} j_{l}\left(k_{f} r\right) R_{n l}(r)
$$

where $j_{l}\left(k_{f} r\right)$ is the spherical Bessel function Arfken et al., 2012 Drake and Cassar, 2006). Fig. 7 shows $\left|f_{n l}\left(k_{f}\right)\right|$ calculated for different radial atomic wave functions

$$
R_{n l}(r) \propto e^{-r / n a}\left(\frac{2 r}{n a}\right)^{l} L_{n-l-1}^{2 l+1}\left(\frac{2 r}{n a}\right)
$$

with associated Laguerre polynomial $L$ and characteristic orbital radius $a . f_{n l}\left(k_{f}\right)$ then can be expressed in analytic closed form

$$
f_{n l}\left(k_{f}\right) \propto \frac{(-i x)^{l}}{\left(x^{2}+1\right)^{l+2}} C_{n-l-1}^{l+1}\left(\frac{x^{2}-1}{x^{2}+1}\right),
$$

where $x=n k_{f} a$ and $C$ is a Gegenbauer polynomial (Drake and Cassar, 2006).

Overall, $\left|f_{n l}\left(k_{f}\right)\right|$ falls off with increasing $k_{f} \propto \sqrt{E_{k i n}}$ and develops $n-l-1$ sharp minima (corresponding to the $n-l-1$ nodes of the Gegenbauer polynomial), which leads to a severe suppression of photoemission intensity at distinct photon energies. These minima are very similar to the so called "Cooper minima" and we will name them as such in the following (Cooper, 1962, Fano and Cooper, 1968).

With increasing $k_{f}$, the final state plane wave - represented by the spherical Bessel function in the integral of Eq. 45 - oscillates and changes sign more rapidly in space. This leads to more cancellation effects for increasing $k_{f}$, and $\left|f_{n l}\left(k_{f}\right)\right|$ consequently vanishes for $k_{f} \rightarrow \infty$. The Cooper minima form at low $k_{f}$, where the final state plane wave (i.e. the spherical Bessel function $j_{l}\left(k_{f} r\right)$ ) changes its sign on a length scale of $\mathcal{O}(a)$. On the same length scale, the orbital wave $R_{n l}(r)$ decays and likewise exhibits a total of $n-l-1$ nodes. The interplay of sign changes of both waves so leads to the $n-l-1$ nodes in $\left|f_{n l}\left(k_{f}\right)\right|$ at distinct momenta $k_{f}$, or equivalently at distinct kinetic energies $E_{k i n}$.

According to Eq. 41, the radial cross section of all orbital contributions to a given electronic state combine and interfere according to their relative phases, resulting in quite complex expressions of the total photoemission intensity. If these orbitals are all of the same type, their radial cross section contributions factor out and can be directly measured by photon energy dependent experiments - given that the final state penetration depth can be neglected $(\lambda \rightarrow \infty)$ and the photon flux has been carefully calibrated ${ }^{6}$. Recently, the feasibility of this idea has been shown by Weiß et al. (2015) for PTCDA molecules with $p$-type electronic emitters, and their data has been successfully modeled by a similar formalism. One drawback however is the lack of information in the small $k_{f}$ regime, which is not accessible by photoemission. This prevents an unbiased inversion of Eq. 45 to recover the radial wave function directly from the data.

\footnotetext{
6 A mere normalization of the photoemission data to a standard reference signal like the absorption current of an upstream gold mesh is typically not sufficient but an absolute calibration of the photon flux is necessary.
} 


\section{G. Sample rotations}

In a typical ARPES experiment with a $2 \mathrm{D}$ detector, only a certain interval of angles $\theta_{k}$ at a given $\phi_{k}$ can be sampled at a time, determined by the acceptance angle of the analyzer and the orientation $\phi_{k}$ of the entrance slit. Due to this limitation, the sample typically has to be rotated to access the complete $\boldsymbol{k}$-space information of the material.

This rotation can be expressed in terms of a rotation matrix $\mathcal{R}(\theta, \beta, \phi)$, which rotates the sample according to three angles $\theta, \beta$ and $\phi$ around the $x$-, $y$ - and $z$-axis, respectively ${ }^{7}$. In the fixed coordinate system of the experiment, the atomic orbitals consequently transform according to a unitary transformation $U(\theta, \beta, \phi)$, which rotates the orbitals according to $\mathcal{R}(\theta, \beta, \phi)$. Neglecting $\lambda$ and $\boldsymbol{k}_{h \nu}$, the matrix element $M^{n l m}\left(\boldsymbol{k}_{f}\right)$ thus becomes a function of $\theta, \beta$, and $\phi$ and consequently transforms as

$$
\begin{aligned}
M_{\boldsymbol{k}_{f}}^{n l m} & \rightarrow M_{\boldsymbol{k}_{f}}^{n l m^{\prime}}(\theta, \beta, \phi) \\
& =\left\langle\boldsymbol{k}_{f}|U(\theta, \beta, \phi)| n l m\right\rangle \\
& =\sum_{m^{\prime}=-l}^{l}\left\langle\boldsymbol{k}_{f} \mid n l m^{\prime}\right\rangle\left\langle n l m^{\prime}|U(\theta, \beta, \phi)| n l m\right\rangle \\
& =\sum_{m^{\prime}=-l}^{l} D_{m^{\prime} m}^{l}(\theta, \beta, \phi)\left\langle\boldsymbol{k}_{f} \mid n l m^{\prime}\right\rangle \\
& =\sum_{m^{\prime}=-l}^{l} D_{m^{\prime} m}^{l}(\theta, \beta, \phi) M_{\boldsymbol{k}_{f}}^{n l m^{\prime}},
\end{aligned}
$$

where the $D_{m^{\prime} m}^{l}(\theta, \beta, \phi)$ are the coefficients of the Wigner D-matrix. These introduce additional orbital contributions to the matrix element, which can lead to a recovery of spectral weight upon rotation. The Wigner D-coefficients are given explicitly for the $s^{-}, p$-, $d$ - and $f$-orbitals in the appendix Sec. $\mathrm{VII}^{8}$

\section{CASE STUDIES}

In the subsequent sections, we will directly apply above results to a number of instructive showcases of increasing complexity. The basis for our considerations will be

\footnotetext{
7 The rotation angles $\theta$ and $\phi$ should not be confused with the angular coordinates $\theta_{k}$ and $\phi_{k}$ of the outgoing photoelectron defined in Eq. 24 and Fig. 3 .

8 Typically, the Wigner D-Matrix is expressed in terms of Euler angles in the $z-x-z$ convention, namely a first rotation $\alpha_{z_{0}}$ about the $z$-axis, a second rotation $\alpha_{x}$ about the $x$-axis and another rotation $\alpha_{z_{1}}$ about the $z$-axis. In general, great care has to be taken to express $\alpha_{z_{0}}, \alpha_{x}$ and $\alpha_{z_{1}}$ in terms of an arbitrary rotation (e.g. $\theta, \beta \phi$ as given here). Such a transformation of rotation $R(\theta, \beta, \phi)$ into a rotation around Euler angles $R\left(\alpha_{z_{1}}, \alpha_{x}, \alpha_{z_{0}}\right)$ can be difficult. For a detailed demonstration see Eberly (2008).
}

Eq. 20 and its tight binding approximation Eq. 41, If not otherwise noted, we will neglect the photon momentum $\boldsymbol{k}_{h \nu}$ as well as the influence of the finite penetration depth $\lambda$ on the final state. Further, we will often times assume measurements within small photon energy intervals $h \nu$ where the radial cross-section is assumed to be constant. The simulated ARPES intensity plots shown here were calculated with Mathematica (Wolfram Research, 2016).

\section{A. The linear chain - band formation in a periodic system}

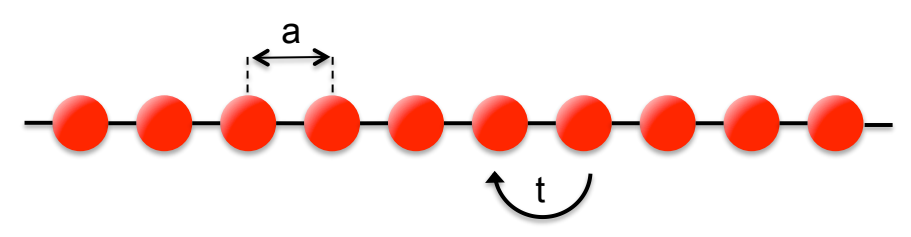

FIG. 8 The mono atomic linear chain.

Let us start with a simplistic but instructive model, the mono atomic linear chain. It consists of $N$ atoms on sites $\boldsymbol{R}_{j}$ with $j=1 \ldots N$ and spaced by the lattice constant $a$. Each atom contributes one atomic orbital $\left|\boldsymbol{R}_{j}, j\right\rangle$, coupled to its adjacent neighbors by the hopping parameter $t$. In the basis $\left\{\left|\boldsymbol{R}_{j}, j\right\rangle\right\}$, the tight binding Hamiltonian of this chain can be written as an $N \times N$ tridiagonal Toeplitz matrix

$$
H=\left(\begin{array}{cccccc}
\varepsilon & t & & \cdots & & 0 \\
t & \varepsilon & t & & & \\
& t & \varepsilon & t & & \vdots \\
\vdots & & \ddots & \ddots & \ddots & \\
& & & t & \varepsilon & t \\
0 & & \ldots & & t & \varepsilon
\end{array}\right)
$$

with eigenvalues (Noschese et al. 2013)

$$
\varepsilon_{\kappa}=\varepsilon+2 t \cos \left(\frac{\kappa}{N+1} \pi\right) \quad \kappa=1 \ldots N
$$

and eigenvectors

$$
|\boldsymbol{R}, \kappa\rangle=\sum_{j=1}^{N} \sin \left(\frac{\kappa j}{N+1} \pi\right)\left|\boldsymbol{R}_{j}, j\right\rangle \quad \kappa=1 \ldots N
$$

The photoemission matrix element of the eigenstates is proportional to their respective Fourier transforms, i.e. 


$$
\begin{aligned}
\left\langle\boldsymbol{k}_{f} \mid \boldsymbol{R}, \kappa\right\rangle & \propto \sum_{j=1}^{N} \sin \left(\frac{\kappa j}{N+1} \pi\right)\left\langle\boldsymbol{k}_{f} \mid \boldsymbol{R}_{j}, j\right\rangle \\
& =\sum_{j=1}^{N} \sin \left(\frac{\kappa j}{N+1} \pi\right) e^{-i \boldsymbol{k}_{f} \cdot \boldsymbol{R}_{j}}\left\langle\boldsymbol{k}_{f} \mid \mathbf{0}, j\right\rangle \\
& =\left\langle\boldsymbol{k}_{f} \mid \mathbf{0}, s\right\rangle \sum_{j=1}^{N} \sin \left(\frac{\kappa j}{N+1} \pi\right) e^{-i k_{f x} x_{j}} \\
& \propto \sum_{j=1}^{N} \sin \left(\frac{\kappa j}{N+1} \pi\right) e^{-i k_{f x} x_{j}}
\end{aligned}
$$

where $x_{j}=a \cdot j$. All atoms are assumed to contribute one $s$-orbital $|\mathbf{0}, s\rangle$ and $\left\langle\boldsymbol{k}_{f} \mid \mathbf{0}, s\right\rangle$ is thus isotropic.

In order to model the spectral contributions, we make use of the noninteracting limit $\left|\left\langle\Psi_{f}^{N-1}\left|\hat{c}_{\boldsymbol{k}}\right| \Psi_{i}^{N}\right\rangle\right|^{2} \delta(\omega-$ $\left.E_{f}^{N-1}+E_{i}^{N}\right) \rightarrow \delta\left(\omega-\varepsilon_{\boldsymbol{k}}\right)$, convoluted by a Gaussian to account for intrinsic and experimental broadening:

$$
G\left(\boldsymbol{k}, \omega-\varepsilon_{\boldsymbol{k}}\right) \sim \frac{1}{\sqrt{2 \pi} \sigma} e^{-\frac{\left(\omega-\varepsilon_{\boldsymbol{k}}\right)^{2}}{2 \sigma^{2}}} .
$$

According to Eq. 8, the ARPES signal of the linear chain is then calculated as

$$
w_{f i}=\sum_{\kappa}\left|\left\langle\boldsymbol{k}_{f} \mid \boldsymbol{R}, \kappa\right\rangle\right|^{2} G_{\kappa}\left(\omega-\varepsilon_{\kappa}\right),
$$

where we replaced the electron momentum $\boldsymbol{k}$ by the subscript $\kappa$ to underline the discrete nature of the finite system.

Calculation results for chains of various lengths are shown in Fig. 9. From chain lengths $N \gtrsim 5$ we can observe the formation of bands. Formally, an increasing length of the chain leads to an increasing number of eigenstates localizing in $k$-space. The constructive and destructive interference of the photoelectrons of all atomic emitters leads to sharper and sharper photoemission maxima in reciprocal space and a consequent formation of electronic bands (Henk, 2002), which can be observed in ARPES experiments on finite systems (Berkebile et al., 2008; Koller et al., 2007, Mugarza et al., 2003, Puschnig and Lueftner, 2015).

\section{B. The linear random chain - the effect of temperature}

To mimic the effect of temperature $T$, we assume the atoms to oscillate around their equilibrium position $\mu_{j}$ according to

$$
P_{j}\left(x_{j}\right)=\frac{1}{\sqrt{2 \pi} \sigma_{j}} e^{-\frac{\left(x_{j}-\mu_{j}\right)}{2 \sigma_{j}^{2}}}
$$

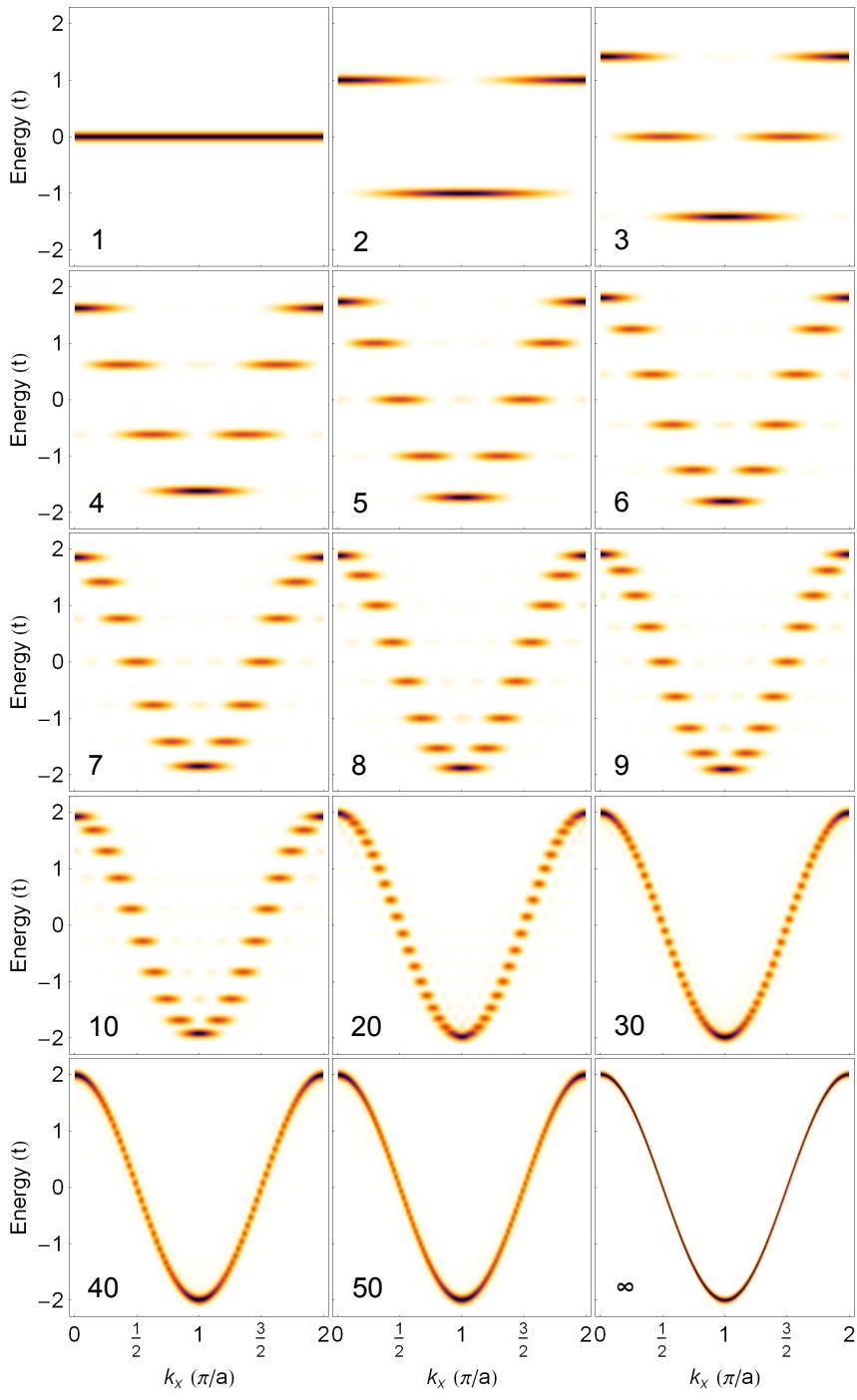

FIG. 9 Band formation calculated according to Eq. 54 for linear chains of different length $N$. The square root of intensity is shown for better contrast.

where $\sigma_{j}=\left\langle x_{j}^{2}\right\rangle \equiv \sigma_{T}$ is the longitudinal standard deviation of atom $j$. Assuming negligible temperature dependence of the Hamiltonian, Eq. 52 becomes

$$
\begin{aligned}
& \left\langle\boldsymbol{k}_{f} \mid \boldsymbol{R}, \kappa\right\rangle \propto \overline{\sum_{j=1}^{N} \sin \left(\frac{\kappa j}{N+1} \pi\right) e^{-i k_{f x} x_{j}}} \\
& \propto \sum_{j=1}^{N} \sin \left(\frac{\kappa j}{N+1} \pi\right) \overline{e^{-i k_{f x} x_{j}}},
\end{aligned}
$$

where the bar denotes the thermal average. We find

$$
\begin{aligned}
\overline{e^{-i k_{f x} x_{j}}} & =\int_{-\infty}^{\infty} d x_{j} P_{j}\left(x_{j}\right) e^{-i k_{f x} x_{j}} \\
& =e^{-i k_{f x} \mu_{j}} e^{-\frac{1}{2} k_{f x}^{2} \sigma_{T}^{2}},
\end{aligned}
$$


and thus

$$
\left\langle\boldsymbol{k}_{f} \mid \boldsymbol{R}, \kappa\right\rangle \propto e^{-\frac{1}{2} k_{f x}^{2} \sigma_{T}^{2}} \sum_{j=1}^{N} \sin \left(\frac{\kappa j}{N+1} \pi\right) e^{-i k_{f x} \mu_{j}}
$$

where $\left|e^{-\frac{1}{2} k_{f x}^{2} \sigma_{T}^{2}}\right|^{2}:=W(T)$ is the well known temperature dependent Debye-Waller factor, which phenomenologically describes the ratio in between the direct and the indirect transitions in the photoemission process (Hussain et al. 1980; Papp et al., 2011; Plucinski et al., 2008 Wagner et al., 1977; White et al. , 1987). The $k$ conserving direct transitions are suppressed for increasing $k_{f}$ due to the loss of short-range order with temperature. At the same time, indirect transitions involving phonon scattering get stronger and result in a "smearing" of the dispersive features. The photoemission signal thus becomes density of states like at higher temperatures and higher $k_{f}$ ("XPS limit"). For a quantitative description of these effects however, more accurate microscopic models have been developed (Braun et al. $, 1992,2013$, Gray et al., 2012, 2011; Larsson and Pendry, 1981).

\section{The linear Fibonacci chain - band formation in a quasi crystal}

To demonstrate how band formation effectively can take place over very small length scales and without need for translational invariance, we quickly discuss the case of a one dimensional quasi crystal. In a quasi crystal, long range periodicity is absent but the system still remains ordered. A well studied example is the Fibonacci chain (Ghosh and Karmakar, 1999, Gumbs and Ali, 1989, S. A. Ketabi and N. Shahtahmasebi, 2005, Sólyom, 2007; Zhong et al. , 1991). It consists of a chain of atoms whose spacing is determined by the Fibonacci sequence $\{A, B, A B, B A B, A B B A B, B A B A B B A B, \ldots\}$. Because of the lack of periodicity, its Fourier components are densely distributed, but still exhibit sharp features. These features eventually can lead to band like distributed spectral weight in ARPES. The position of the $j$ th atom of the Fibonacci chain can be constructed as

$$
x_{j}=a\left(j-1+\frac{1}{\tau}\left\lfloor\frac{j}{\tau}\right\rfloor\right) \quad j=1 \ldots N
$$

where $\tau=\frac{1+\sqrt{5}}{2}$ is the golden ratio and $\lfloor x\rfloor$ is the floor, i.e. the integer part of $x$. The distance in between atoms is thus either $a$ or $a(1+1 / \tau)$. We further assume - for simplicity and without changing the main conclusion of this section - that the hopping integrals in between atoms regardless of their distance remains $t$, and the eigenvalues
(Eq. 50) and eigenvectors (Eq. 51) remain the ones of the linear chain. In analogy, the angular distribution of photoelectrons is determined by the Fourier transform of Eq. 52, where $x_{j}$ now takes the form of Eq. 59 .

Calculation results for a spherical wave function $|\mathbf{0}, s\rangle$ are shown in Fig. 10, plotted as a function of the average lattice spacing

$$
a^{*}=\lim _{N \rightarrow \infty} \frac{x_{N}}{N-1}=\frac{1}{2}(5-\sqrt{5}) a .
$$

Already for chain lengths of $N \gtrsim 5$ atoms, one recognizes the formation of pseudo bands which get sharper and sharper with increasing length. However, the interference terms of all atoms do not single out one contribution. In fact, a whole multitude of band-like features appears, mirroring the distribution of Fourier components within the chain. Experimentally, such an effect has been demonstrated e.g. in the $2 \mathrm{D}$ decagonal quasi crystal $\mathrm{Al}_{71.8} \mathrm{Ni}_{14.8} \mathrm{Co}_{13.4}$ (Rotenberg et al. 2000 ).

\section{The linear chain in the bulk limit}

Let us now directly approach the linear chain from the infinite case $N \rightarrow \infty$, explicitly taking into account the long range periodicity of the lattice. The tight binding solution can now be directly written as

$$
\left|\Psi_{\kappa \boldsymbol{k}}\right\rangle=\sum_{\boldsymbol{R}_{j}} e^{i \boldsymbol{k} \cdot \boldsymbol{R}_{j}}\left|\boldsymbol{R}_{j}, \kappa\right\rangle
$$

with eigenvalues $\varepsilon_{\boldsymbol{k}}=\varepsilon_{\boldsymbol{k}+\boldsymbol{K}}=\varepsilon+2 t \cos \left(a k_{x}\right)$. The ARPES intensity is thus distributed as the Fourier transform of $\left|\Psi_{\kappa \boldsymbol{k}}\right\rangle$

$$
M_{\boldsymbol{k}_{f} \boldsymbol{k}}^{\kappa} \propto\left\langle\boldsymbol{k}_{f} \mid \Psi_{\kappa \boldsymbol{k}}\right\rangle=\sqrt{2 \pi} \frac{N}{L} \delta\left(\boldsymbol{k}+\boldsymbol{K}-\boldsymbol{k}_{f}\right)\left\langle\boldsymbol{k}_{f} \mid \mathbf{0}, \kappa\right\rangle,
$$

which depends only on the spatial distribution of $\langle\boldsymbol{r} \mid \mathbf{0}, \kappa\rangle$. The corresponding spectral weight is given by

$$
\begin{aligned}
w_{f i} & \propto \sum_{\boldsymbol{k}}\left|\left\langle\boldsymbol{k}_{f} \mid \mathbf{0}, \kappa\right\rangle\right|^{2} \delta\left(\boldsymbol{k}+\boldsymbol{K}-\boldsymbol{k}_{f}\right) G\left(\boldsymbol{k}, \omega-\varepsilon_{\boldsymbol{k}}\right) \\
& =\left|\left\langle\boldsymbol{k}_{f} \mid \mathbf{0}, \kappa\right\rangle\right|^{2} G\left(\boldsymbol{k}_{f}, \omega-\varepsilon_{\boldsymbol{k}_{f}}\right) .
\end{aligned}
$$

Calculations for an $s$-orbital $|\mathbf{0}, s\rangle$ are shown in the bottom right of of Fig. 9 .

\section{E. The diatomic linear chain}

As an instructive generalization of the mono atomic chain, we study the diatomic linear chain with atomic orbitals $\left|\boldsymbol{R}_{A}, A\right\rangle$ and $\left|\boldsymbol{R}_{B}, B\right\rangle$ on lattice sites $\boldsymbol{R}_{A}=$ 


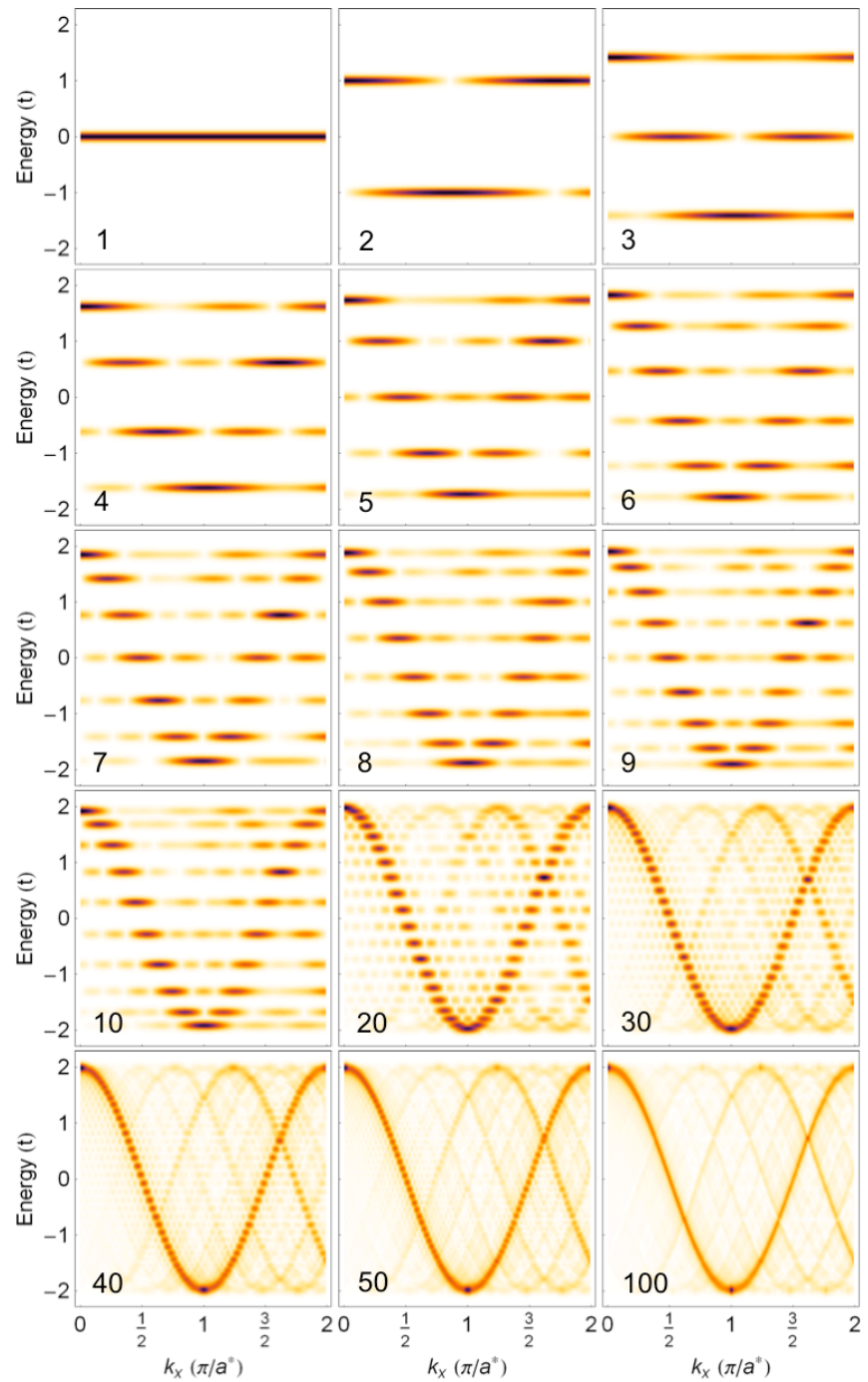

FIG. 10 Band formation calculated according to Eq. 54 for Fibonacci chains of different length $N$. The square root of intensity is shown for better contrast.

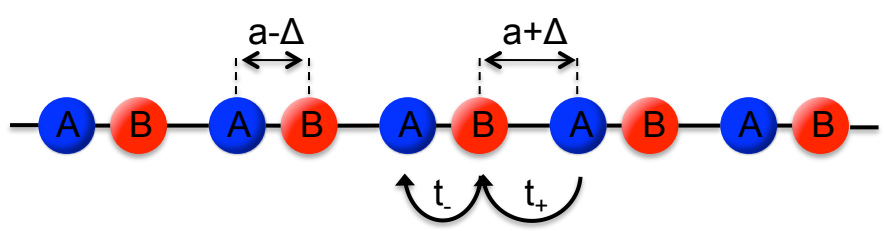

FIG. 11 The diatomic linear chain.

$\frac{a-\Delta}{2}\{-1,0,0\}$ and $\boldsymbol{R}_{B}=\frac{a-\Delta}{2}\{1,0,0\}$ within the primitive unit cell, respectively (Fig. 11). In this basis, the tight binding Hamiltonian reads

$$
H=\left(\begin{array}{cc}
\varepsilon & h_{\mathbf{k}} \\
h_{\mathbf{k}}^{*} & \varepsilon
\end{array}\right)
$$

where $h_{\mathbf{k}}$ describes charge hopping in between sub lat- tices $\mathrm{A}$ and $\mathrm{B}$. The eigenvalues of $H$ are given by $\varepsilon_{\mathbf{k}}^{ \pm}=$ $\varepsilon \pm\left|h_{\mathbf{k}}\right|$, with eigenstates $\left\{c_{A}^{ \pm}, c_{B}^{ \pm}\right\}=\frac{1}{\sqrt{2}}\left\{1, \pm \frac{h_{\mathbf{k}}}{\left|h_{\mathbf{k}}\right|}\right\}$. The tight binding form of the Bloch wave thus reads

$$
\left|\Psi_{ \pm \boldsymbol{k}}\right\rangle=\frac{1}{\sqrt{N}} \sum_{\boldsymbol{R}} e^{i \boldsymbol{k} \boldsymbol{R}}|\boldsymbol{R}, \pm\rangle
$$

where the sum goes over all lattice sites $\boldsymbol{R}$ and \pm labels bonding and anti bonding states

$$
|\boldsymbol{R}, \pm\rangle=\frac{1}{\sqrt{2}}\left(e^{i \boldsymbol{R}_{A} \cdot \boldsymbol{k}}\left|\boldsymbol{R}+\boldsymbol{R}_{A}, A\right\rangle \pm \frac{h_{\mathbf{k}}}{\left|h_{\mathbf{k}}\right|} e^{i \boldsymbol{R}_{B} \cdot \boldsymbol{k}}\left|\boldsymbol{R}+\boldsymbol{R}_{B}, B\right\rangle\right)
$$

In the free electron final state approximation, the photoemission matrix element is expressed by the Fourier transform of the tight binding orbital

$$
\begin{aligned}
M_{\boldsymbol{k}_{f} \boldsymbol{k}}^{ \pm} & \propto\left\langle\boldsymbol{k}_{f} \mid \mathbf{0}, \pm\right\rangle \\
& =\left\langle\boldsymbol { k } _ { f } \left|\frac{1}{\sqrt{2}}\left(e^{i \boldsymbol{k} \cdot \boldsymbol{R}_{A}}\left|\boldsymbol{R}_{A}, A\right\rangle \pm \frac{h_{\mathbf{k}}}{\left|h_{\mathbf{k}}\right|} e^{i \boldsymbol{k} \cdot \boldsymbol{R}_{B}}\left|\boldsymbol{R}_{B}, B\right\rangle\right)\right.\right. \\
& =\frac{1}{\sqrt{2}}\left(e^{i \boldsymbol{k} \cdot \boldsymbol{R}_{A}}\left\langle\boldsymbol{k}_{f} \mid \boldsymbol{R}_{A}, A\right\rangle \pm \frac{h_{\mathbf{k}}}{\left|h_{\mathbf{k}}\right|} e^{i \boldsymbol{k} \cdot \boldsymbol{R}_{B}}\left\langle\boldsymbol{k}_{f} \mid \boldsymbol{R}_{B}, B\right\rangle\right) \\
& =\frac{1}{\sqrt{2}}\left(e^{i \boldsymbol{k} \cdot \boldsymbol{R}_{A}} e^{-i \boldsymbol{k}_{f} \cdot \boldsymbol{R}_{A}}\left\langle\boldsymbol{k}_{f} \mid \mathbf{0}, A\right\rangle\right. \\
& \left. \pm \frac{h_{\mathbf{k}}}{\left|h_{\mathbf{k}}\right|} e^{i \boldsymbol{k} \cdot \boldsymbol{R}_{B}} e^{-i \boldsymbol{k}_{f} \cdot \boldsymbol{R}_{B}}\left\langle\boldsymbol{k}_{f} \mid \mathbf{0}, B\right\rangle\right) .
\end{aligned}
$$

Taking into account the in plane momentum conservation $\delta\left(\boldsymbol{k}_{\|}+\boldsymbol{K}_{\|}-\boldsymbol{k}_{f \|}\right)$ in the Brillouin zone summation of Eq. 8 and writing $|\mathbf{0}, A(B)\rangle \equiv|A(B)\rangle$, the result simplifies considerably and the photoemission intensity reads

$$
\begin{aligned}
w_{f i}^{ \pm} & \propto\left|\frac{1}{\sqrt{2}}\left(\left\langle\boldsymbol{k}_{f} \mid A\right\rangle \pm \frac{h_{\boldsymbol{k}_{f}}}{\left|h_{\boldsymbol{k}_{f}}\right|}\left\langle\boldsymbol{k}_{f} \mid B\right\rangle\right)\right|^{2} \\
& =\frac{1}{2}\left(\left|\left\langle\boldsymbol{k}_{f} \mid A\right\rangle\right|^{2}+\left|\left\langle\boldsymbol{k}_{f} \mid B\right\rangle\right|^{2}\right. \\
& \left. \pm 2 \Re\left\{\left\langle\boldsymbol{k}_{f} \mid A\right\rangle\left\langle B \mid \boldsymbol{k}_{f}\right\rangle \frac{h_{\boldsymbol{k}_{f}}}{\left|h_{\boldsymbol{k}_{f}}\right|}\right\}\right)
\end{aligned}
$$

Let us first assume orbitals $\mathrm{A}$ and $\mathrm{B}$ to be identical, i.e. $|A\rangle=|B\rangle$. If the distance in between site $\mathrm{A}$ and $\mathrm{B}$ is alternating in between $a+\Delta$ and $a-\Delta$, then $h_{k}=$ $-t_{-} e^{-i(a-\Delta) k_{x}}-t_{+} e^{i(a+\Delta) k_{x}}$ is complex, $\left|\Re h_{\boldsymbol{k}_{f}}\right|<\left|h_{\boldsymbol{k}_{f}}\right|$ and the photoemission intensity is given by

$$
w_{f i}^{ \pm} \propto\left|\left\langle\boldsymbol{k}_{f} \mid A\right\rangle\right|^{2}\left(1 \pm \frac{\Re h_{\boldsymbol{k}_{f}}}{\left|h_{\boldsymbol{k}_{f}}\right|}\right) \neq 0
$$

Assuming charge hopping in between $\mathrm{A}$ and $\mathrm{B}$ is $t_{-} \sim$ $t_{+} \sim t$, then $h_{\mathbf{k}}=-2 t e^{i \Delta k_{x}} \cos \left[a k_{x}\right]$ and we obtain 


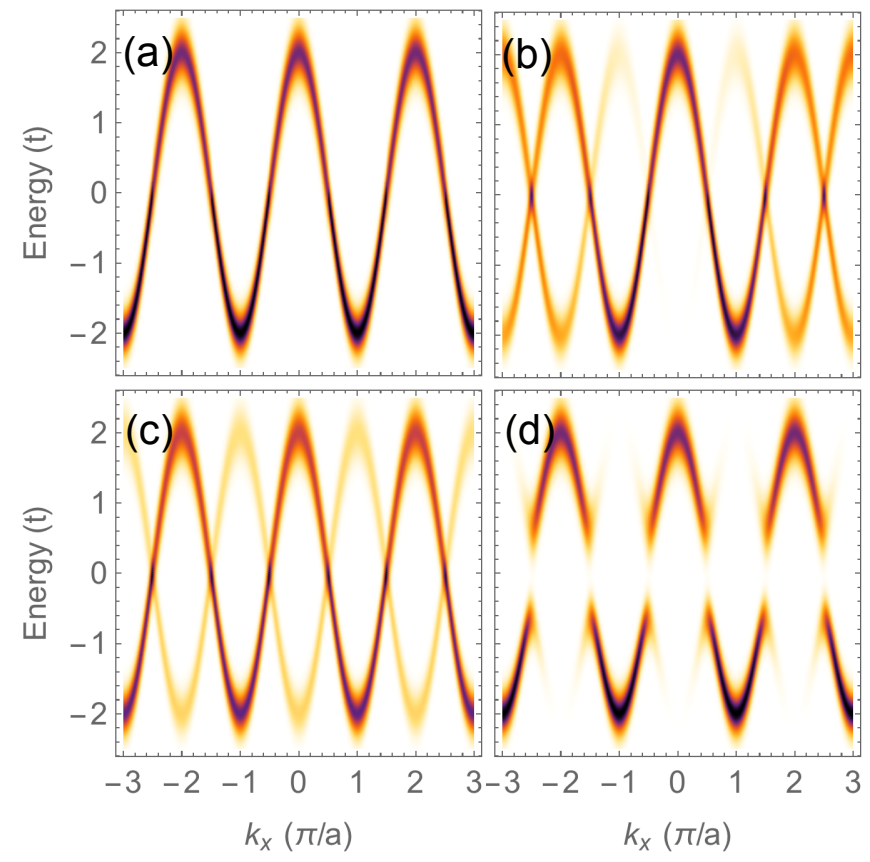

FIG. 12 ARPES intensity distribution in the diatomic chain: (a) the monoatomic chain limit $t_{-}=t_{+}, \Delta=0$ and $|A\rangle=$ $|B\rangle$. (b) dimerization $\Delta \neq 0$, (c) inequivalent orbitals $|A\rangle \neq$ $|B\rangle$, (d) gap formation due to hybridization $t_{+} \neq t_{-}$

$$
w_{f i}^{ \pm} \propto\left|\left\langle\boldsymbol{k}_{f} \mid A\right\rangle\right|^{2}\left(1 \pm \operatorname{sgn}\left(\cos \left[a k_{f x}\right]\right) \cos \left[\Delta k_{f x}\right]\right) .
$$

The intensity ratio in between the low and high intensity band is then given by

$$
\frac{w_{f i}^{\min }}{w_{f i}^{\max }}=\frac{1-\cos \left[\Delta k_{f x}\right]}{1+\cos \left[\Delta k_{f x}\right]} .
$$

If $\Delta=0$, then $w_{f i}^{ \pm} \propto \frac{1}{2}\left(1 \pm \operatorname{sgn}\left(\cos \left[a k_{f x}\right]\right)\right)$, i.e. a rectangular function which flips spectral weight in between states $|+\rangle$ and $|-\rangle$ whenever the cosine switches sign, i.e. with periodicity $2 \pi / a$ and thus at the double periodicity of $\varepsilon_{k}$ (which varies with periodicity $\pi / a$ ). Hence, one of the two eigensolutions $|+\rangle$ or $|-\rangle$ is always completely suppressed at a time with respect to the other and the primitive symmetry of a system with only one orbital $|A\rangle$ per unit cell - namely the mono atomic linear chain discussed above - is restored (see Fig. 12 a).

If we allow for $\Delta \neq 0$, i.e. the breaking of translational symmetry concerning sites $\mathrm{A}$ and $\mathrm{B}$ due to dimerization, then $w_{f i}$ still varies with $2 \pi / a$ and $\varepsilon_{k}$ still with $\pi / a$. However, $w_{f i}$ is now additionally modulated by a beating term $\cos \left[\Delta k_{x}\right]$. Thus, the intensity is not strictly zero anymore for $k_{x} \neq 0$ and a shadow/Umlapp band can be observed (see Fig. 12 b).

Let us now assume the chain to be equally spaced, $\Delta=0$ and $t_{-}=t_{+}=t$, but orbitals $\mathrm{A}$ and $\mathrm{B}$ to be distinct, i.e. $|A\rangle \neq|B\rangle$. The intensity ratio in between the low and high intensity band is then given by

$$
\frac{I_{\min }}{I_{\max }}=\frac{\left(\left\langle\boldsymbol{k}_{f} \mid A\right\rangle-\left\langle\boldsymbol{k}_{f} \mid B\right\rangle\right)^{2}}{\left(\left\langle\boldsymbol{k}_{f} \mid A\right\rangle+\left\langle\boldsymbol{k}_{f} \mid B\right\rangle\right)^{2}} .
$$

Clearly, the doubling of the unit cell via the inequivalence of $\mathrm{A}$ and $\mathrm{B}$ introduces nonzero spectral weight of an Umklapp band (see Fig. 12 c). The intensity ratio in between both bands along $k_{x}$ then can be interpreted as a measure of the strength of the Fourier component of the electron density which lowers the translational symmetry of a system with ordering vector $2 \pi / a$ to one with ordering vector $\pi / a$ - often times called the Umklapp potential.

Finally, if $t_{+} \neq t_{-}$, the main and the Umklapp band hybridize and a gap forms (see Fig. 12 d).

\section{F. Orbital mapping of ordered molecules}

Photoemission on adsorbed molecules is in principle a matter of long standing interest (Gadzuk, 1974a b, 1975, Grobman, 1978, Mahan, 1970 Schaich and Ashcroft, 1971: Ueno et al., 1997, 1993). However, it was due to big advances in instrumentation during the last decade that large portions of the photoemission hemisphere became readily accessible and the full orbital wave function of the molecules could be determined. This technique - often referred to as orbital tomography - exploits the fact that single molecules ordered on a substrate act as coherent emitters of photoelectrons (Berkebile et al. 2008 , Bradshaw and Woodruff, 2015; Dauth et al., 2011; Kera et al., 2006; Koller et al., 2007; Lueftner et al., 2014; Nguyen et al., 2015; Puschnig et al., 2009; Puschnig and Lueftner, 2015; Puschnig et al., 2011; Wießner et al., 2014; Wiessner et al., 2012; Ziroff et al. , 2010). The photo electron distribution then provides information on the spatial extent of the molecular orbitals.

Two benchmark examples which have been studied recently are sexiphenyl and pentacene (Puschnig et al. 2009). Their respective structures (hydrogen atoms are omitted) are depicted in Fig. 13 and 14 respectively. Both molecules are aromatic systems, i.e. the molecular states close to the Fermi level are primarily formed from a linear combination $|\boldsymbol{R}, \kappa\rangle \sim \sum_{\boldsymbol{R}_{j}} c_{j}^{\kappa}\left|\boldsymbol{R}_{j}, p_{z}\right\rangle$ of C $2 p_{z}$ orbitals, located at each carbon site $\boldsymbol{R}_{j}$ of the molecule. In the Hückel approximation, we assume a hopping parameter $t$ only in between adjacent carbon atoms. The coefficients $c_{j}^{\kappa}$ can then be determined from the orthogonalized Eigenset of the tight binding Hamiltonian.

For the example of sexiphenyl, we have to diagonalize a $36 \times 36$ matrix of the form 

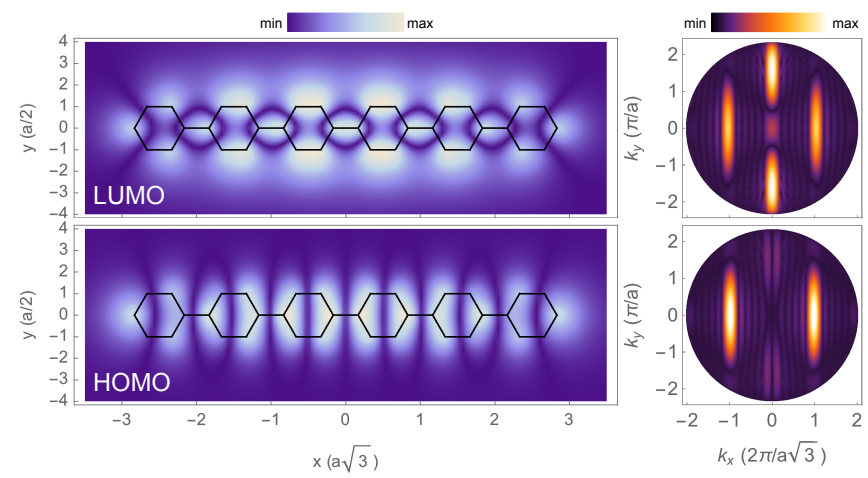

FIG. 13 Tight binding calculation (left) and corresponding stereographic projection of the angular distribution of photoelectrons of sexiphenyl calculated with $a=2.42 \AA$ and $E_{k i n}=30 \mathrm{eV}$ (right).
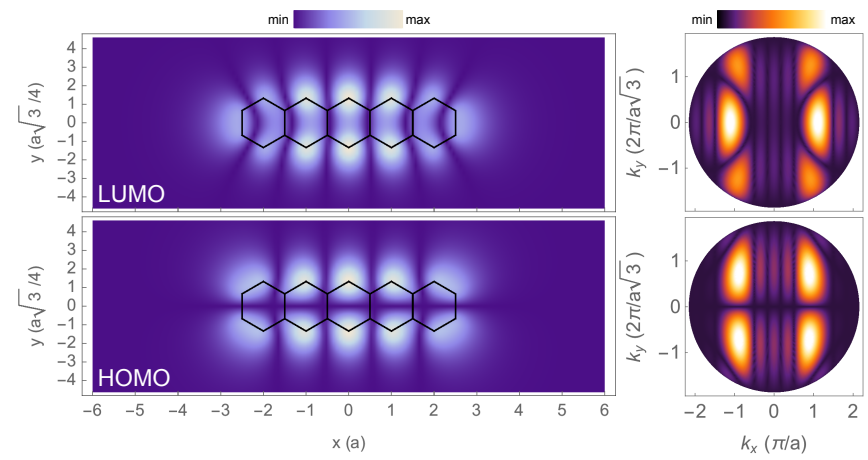

FIG. 14 Tight binding calculation (left) and corresponding stereographic projection of the angular distribution of photoelectrons of pentacene calculated with $a=2.42 \AA$ and $E_{\text {kin }}=30 \mathrm{eV}$ (right).

$$
H=\left(\begin{array}{cccccc}
H_{0} & H_{1} & 0 & 0 & 0 & 0 \\
H_{1}^{T} & H_{0} & H_{1} & 0 & 0 & 0 \\
0 & H_{1}^{T} & H_{0} & H_{1} & 0 & 0 \\
0 & 0 & H_{1}^{T} & H_{0} & H_{1} & 0 \\
0 & 0 & 0 & H_{1}^{T} & H_{0} & H_{1} \\
0 & 0 & 0 & 0 & H_{1}^{T} & H_{0}
\end{array}\right)
$$

where

$$
H_{0}=\left(\begin{array}{cccccc}
\varepsilon & t & 0 & 0 & 0 & t \\
t & \varepsilon & t & 0 & 0 & 0 \\
0 & t & \varepsilon & t & 0 & 0 \\
0 & 0 & t & \varepsilon & t & 0 \\
0 & 0 & 0 & t & \varepsilon & t \\
t & 0 & 0 & 0 & t & \varepsilon
\end{array}\right)
$$

is the Hamiltonian of the individual benzene cycles forming the molecule, which are interconnected through

$$
H_{1}=\left(\begin{array}{cccccc}
0 & 0 & 0 & 0 & 0 & 0 \\
0 & 0 & 0 & 0 & 0 & 0 \\
0 & 0 & 0 & 0 & 0 & 0 \\
t & 0 & 0 & 0 & 0 & 0 \\
0 & 0 & 0 & 0 & 0 & 0 \\
0 & 0 & 0 & 0 & 0 & 0
\end{array}\right) .
$$

This computational task can be greatly reduced by block factorization of the Hamiltonian, exploiting the $D_{2 h}$ symmetry of the molecule (Cotton, 1990). Sorting the result according to increasing Eigenvalues of $H$ and filling up the available states with a total of 36 electrons, we identify the highest occupied (HOMO) and lowest unoccupied molecular orbital (LUMO). Omitting the pseudo momentum conservation as well as the polarization dependent terms, the photoelectron distribution according to Eq. 41 can be written as

$$
M_{\boldsymbol{k}_{f} \boldsymbol{k}}^{\kappa} \propto\left\langle\boldsymbol{k}_{f} \mid \mathbf{0}, p_{z}\right\rangle \sum_{\boldsymbol{R}_{j}} c_{j}^{\kappa} e^{i \boldsymbol{k}_{f} \cdot \boldsymbol{R}_{j}},
$$

which is independent of $\varepsilon$ and $t$. The Hamiltonian of other aromatic compounds like pentacene and the corresponding calculation of the angular distribution of photoelectrons works in an analogous way.

Calculation results for the HOMO and LUMO of sexiphenyl and pentacene are shown in Fig. 13 and 14 , respectively. The left column shows a planar projection of the modulus square of the molecular wave function superposed to the formal chemical structure. The right column shows the corresponding angular distribution of photoelectrons as emitted into half space. Both the real and the $\boldsymbol{k}$-space representations of the molecular orbitals show good agreement to the experimental results published by Puschnig et al. (2009). For higher accuracy however, the coefficients $c_{j}^{\kappa}$ have to be determined within the framework of more realistic models such as density functional theory.

Recently, experiments on ordered 3,4,9,10perylenetetracarboxylic dianhydride (PTCDA), molecules have been performed using circular polarized light (Wießner et al. 2014 ). Surprisingly, the related dichroism signal shows non-vanishing signatures, despite the absence of distinct chiralities within the molecule. A particularly large circular dichroism asymmetry was found perpendicular to the incident light direction.

According to Fig. 3. a handedness of the experimental geometry is introduced naturally when light source and detector are non-collinear $(\alpha>0)$ and the penetration depth $\lambda$ of the final state is reasonably finite. In view of this handedness, one can argue that some contribution of this signal may come from the finite penetration depth of the free electron final state. According to Eq. 31 the circular dichroism intensity scales as 


$$
\left|M_{\boldsymbol{k}_{f} \boldsymbol{k}}^{\circlearrowright}\right|^{2}-\left|M_{\boldsymbol{k}_{f} \boldsymbol{k}}^{\circlearrowleft}\right|^{2} \propto-\frac{2 \epsilon^{2} k_{f x} \sin \alpha}{\lambda}\left|\left\langle\boldsymbol{k}_{f} \mid \mathbf{0}, \kappa\right\rangle\right|^{2},
$$

where $k_{f x}$ is the direction perpendicular to the scattering plane, i.e. perpendicular to the incoming light direction and along the detected asymmetry.

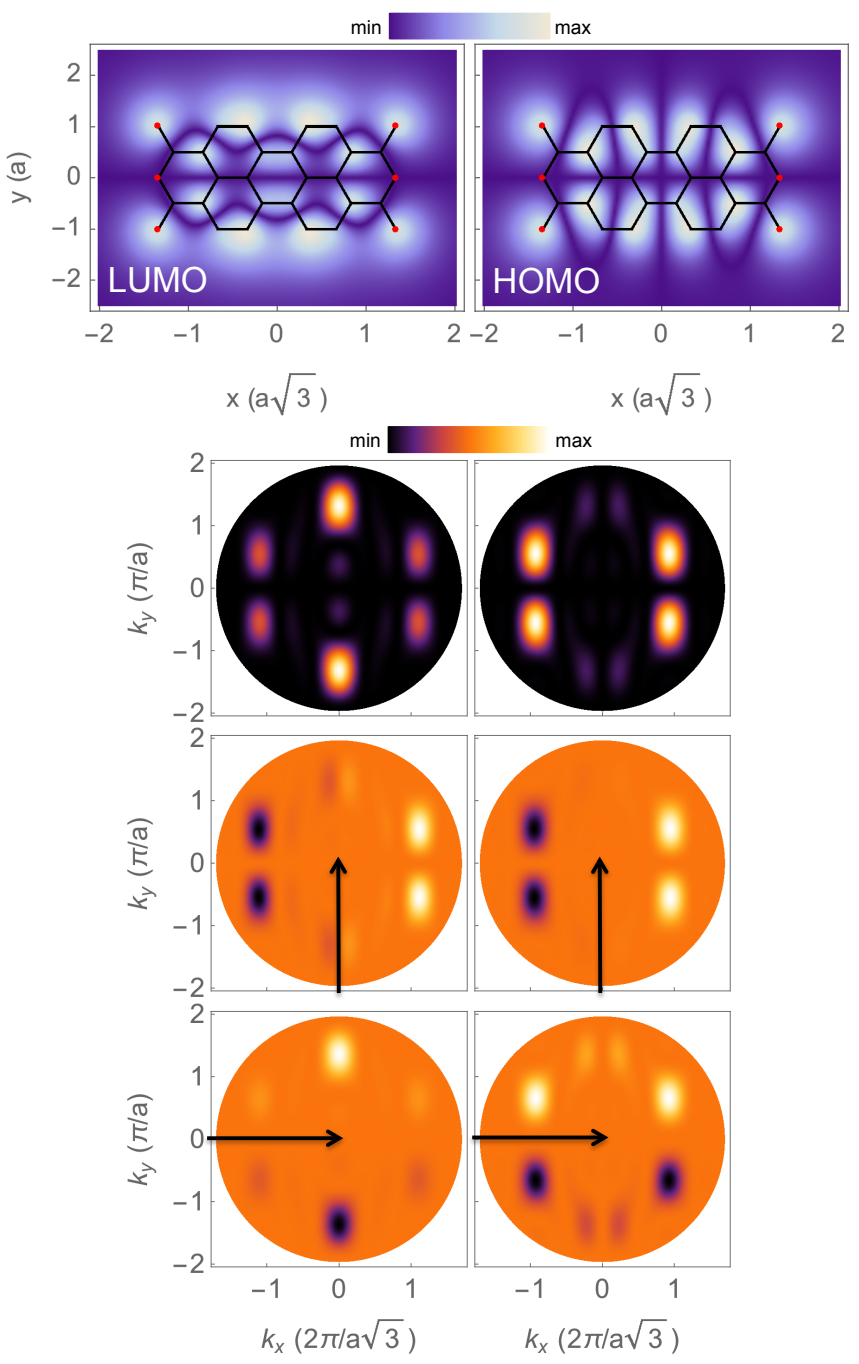

FIG. 15 Tight binding calculation (1st row) and corresponding stereographic projection of the angular distribution of photoelectrons (2nd row) of PTCDA calculated with $a=2.42 \AA$ and $E_{k i n}=27 \mathrm{eV}$. The red disks mark oxygen atoms, hydrogen atoms are omitted. p-type circular dichroism results for samples exposed along the $y z$ (3rd row) and along the $x z$ plane (4th row).

In analogy to sexiphenyl and pentacene, we construct a tight binding Hamiltonian only taking into account the $p_{z}$ orbitals of the carbon and oxygen atoms. For simplicity, we assume all bond lengths to be equal. The molecular structure (omitting hydrogen atoms) is shown in Fig. 15. The $\pi$-bonds in between adjacent carbon atoms are characterized by the hopping parameter $t$. The relative hybridization strength of the carbonyl groups $t_{1} / t$ as well as the hybridization strengths of the ester group $t_{2} / t$ were estimated from average bond dissociation enthalpies: $t_{1} / t \sim 1.5$ and $t_{1} / t \sim 1.35$ (Sanderson, 1976, 1983).

In plane cuts through the real space representations of the HOMO and LUMO are shown in the 1st row of Fig. 15 superposed to the molecular structure. Despite the simplicity of this model, they match the published DFT results reasonably well (Wießner et al., 2014). The 2nd row shows the angular distribution of photoelectrons calculated from Eq. 41 for both HOMO and LUMO, likewise matching the related experiments. In the 3rd row we introduce the asymmetry term of Eq. 31 adapted to light shining in along the $y z$ plane, mimicking the experimentally observed asymmetry. The 4th row shows an analogous calculation for light entering along the $x z$ plane. We also note that the experimental result might be further affected by deviations of the light polarization from their nominal value as discussed in Sec. III.E. The awareness of such effects is thus of primordial importance for the interpretation of dichroism data.

\section{G. Matrix elements in graphene}

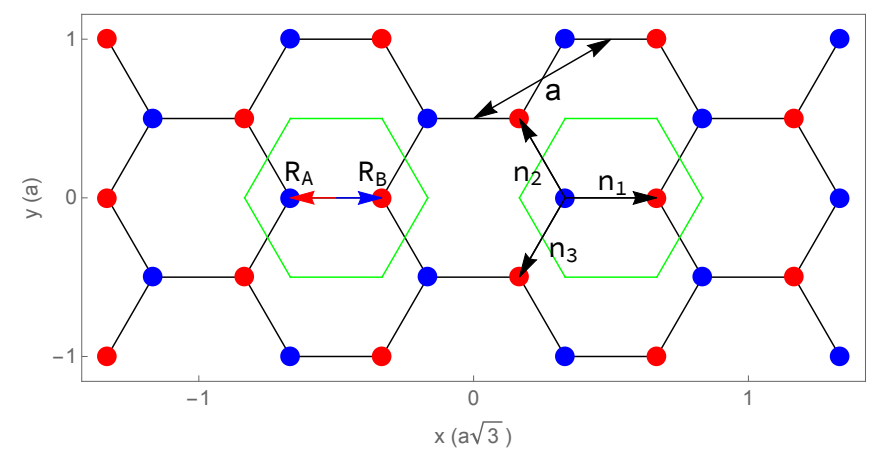

FIG. 16 . Graphene grid showing unit cell (green) and basic vectors. Inequivalent atoms are marked in red and blue.

Let us now turn to a real 2D system: graphene (Bena and Montambaux, 2009, Bostwick et al., 2007; Castro Neto et al., 2009, Hwang et al., 2011; MuchaKruczyński et al. 2008; Shirley et al., 1995). The unit cell of graphene consists of two inequivalent carbon atoms situated at $\boldsymbol{R}_{A}=\frac{a}{2 \sqrt{3}}\{-1,0\}$ and $\boldsymbol{R}_{B}=\frac{a}{2 \sqrt{3}}\{1,0\}$ within the unit cell (see Fig. 16). Each of these carbon atoms contributes one $\mathrm{C} 2 p_{z}$-orbital forming aromatic $\pi$ bonds. The nearest neighbors of carbon atom $A$ are atoms of type $B$ at site $\boldsymbol{n}_{1}=\{a / \sqrt{3}, 0\}$ within the same unit cell and at $\boldsymbol{n}_{2}=\{-a / 2 \sqrt{3}, a / 2\}$ and $\boldsymbol{n}_{3}=\{-a / 2 \sqrt{3},-a / 2\}$ within adjacent unit cells. In the basis $\left\{\left|R_{A}, p_{z}\right\rangle,\left|R_{B}, p_{z}\right\rangle\right\}$, the tight binding Hamil- 
ton reads

$$
H=\left(\begin{array}{cc}
0 & -t f(\boldsymbol{k}) \\
-t f^{*}(\boldsymbol{k}) & 0
\end{array}\right)
$$

where

$$
f(\boldsymbol{k})=e^{i \boldsymbol{n}_{1} \cdot \boldsymbol{k}}+e^{i \boldsymbol{n}_{2} \cdot \boldsymbol{k}}+e^{i \boldsymbol{n}_{3} \cdot \boldsymbol{k}}
$$

describes charge hopping in between $A$ to $B$, and $t$ is the nearest neighbor hopping integral (Bena and Montambaux, 2009). Diagonalizing $H$, we find eigenvalues

$$
\begin{aligned}
\varepsilon_{\boldsymbol{k}}^{ \pm} & = \pm t|f(\boldsymbol{k})| \\
& = \pm t \sqrt{3+2 \cos \left(a k_{y}\right)+4 \cos \left(a \sqrt{3} k_{x} / 2\right) \cos \left(a k_{y} / 2\right)}
\end{aligned}
$$

with eigenvectors $\left\{c_{A}^{ \pm}, c_{B}^{ \pm}\right\}=\frac{1}{\sqrt{2}}\left\{1, \pm e^{i \vartheta_{k}}\right\} . \quad e^{i \vartheta_{k}}=$ $f(\boldsymbol{k}) /|f(\boldsymbol{k})|$ defines the argument of $f(\boldsymbol{k})$, which is plotted in Fig. 17. We can thus write down the tight binding form of the Bloch wave as

$$
\left|\Psi_{ \pm \boldsymbol{k}}\right\rangle=\frac{1}{\sqrt{N}} \sum_{\boldsymbol{R}} e^{i \boldsymbol{k} \cdot R}|\boldsymbol{R}, \pm\rangle
$$

where

$$
|\boldsymbol{R}, \pm\rangle=\frac{1}{\sqrt{2}}\left(e^{i \boldsymbol{k} \cdot \boldsymbol{R}_{A}}\left|\boldsymbol{R}+\boldsymbol{R}_{A}, p_{z}\right\rangle \pm e^{i \vartheta_{\boldsymbol{k}}} e^{i \boldsymbol{k} \cdot \boldsymbol{R}_{B}}\left|\boldsymbol{R}+\boldsymbol{R}_{B}, p_{z}\right\rangle\right.
$$

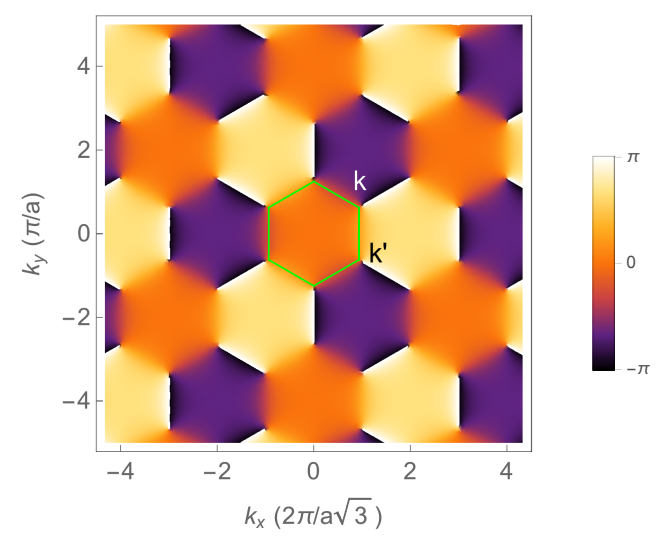

FIG. 17 . Phase $\vartheta_{\boldsymbol{k}}$ of graphene. The green hexagon indicates the first Brillouin zone.

The matrix element can now be directly written as the Fourier transformation of the local Wannier orbital $|\mathbf{0}, \pm\rangle$ and we obtain

$$
\begin{aligned}
M_{\boldsymbol{k}_{f} \boldsymbol{k}}^{ \pm} & \propto\left\langle\boldsymbol{k}_{f} \mid \mathbf{0}, \pm\right\rangle \\
& =\left\langle\boldsymbol { k } _ { f } \left|\frac{1}{\sqrt{2}}\left(e^{i \boldsymbol{k} \cdot \boldsymbol{R}_{A}}\left|\boldsymbol{R}_{A}, p_{z}\right\rangle \pm e^{i \vartheta_{\boldsymbol{k}}} e^{i \boldsymbol{k} \cdot \boldsymbol{R}_{B}}\left|\boldsymbol{R}_{B}, p_{z}\right\rangle\right)\right.\right. \\
& =\frac{1}{\sqrt{2}}\left(e^{i \boldsymbol{k} \cdot \boldsymbol{R}_{A}}\left\langle\boldsymbol{k}_{f} \mid \boldsymbol{R}_{A}, p_{z}\right\rangle \pm e^{i \vartheta_{\boldsymbol{k}}} e^{i \boldsymbol{k} \cdot \boldsymbol{R}_{B}}\left\langle\boldsymbol{k}_{f} \mid \boldsymbol{R}_{B}, p_{z}\right\rangle\right) \\
& =\frac{1}{\sqrt{2}}\left(e^{i \boldsymbol{k} \cdot \boldsymbol{R}_{A}} e^{-i \boldsymbol{k}_{f} \cdot \boldsymbol{R}_{A}} \pm e^{i \vartheta_{\boldsymbol{k}}} e^{i \boldsymbol{k} \cdot \boldsymbol{R}_{B}} e^{-i \boldsymbol{k}_{f} \cdot \boldsymbol{R}_{B}}\right)\left\langle\boldsymbol{k}_{f} \mid \mathbf{0}, p_{z}\right\rangle
\end{aligned}
$$

Taking into account the in plane momentum conservation $\delta\left(\boldsymbol{k}_{\|}+\boldsymbol{K}_{\|}-\boldsymbol{k}_{f \|}\right)$ in the Brillouin zone summation of Eq. 8, the result simplifies to

$$
M_{\boldsymbol{k}_{f} \boldsymbol{k}}^{ \pm} \propto \frac{1}{\sqrt{2}}\left(1 \pm e^{i \vartheta_{\boldsymbol{k}_{f}}}\right)\left\langle\boldsymbol{k}_{f} \mid \mathbf{0}, p_{z}\right\rangle
$$

The ARPES signal therefore vanishes wherever $\left|M_{\boldsymbol{k}_{f} \boldsymbol{k}}^{ \pm}\right|^{2}=1 \pm \cos \vartheta_{\boldsymbol{k}_{f}}$ is zero, i.e. $\vartheta_{\boldsymbol{k}_{f}}= \pm \pi$.

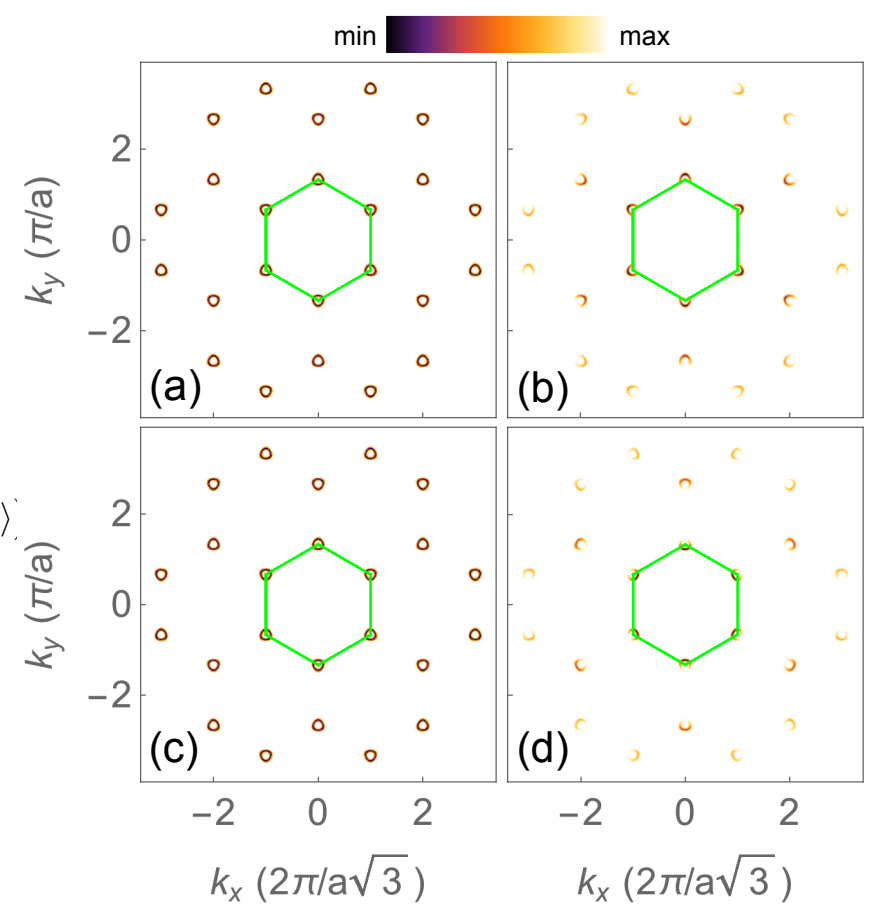

FIG. 18 Stereographic projection of the angular distribution of photoelectrons in graphene with $E_{k i n}=91 \mathrm{eV}$. (a,c) Tight binding spectral function without and with (b,d) matrix elements $\left|M_{\boldsymbol{k}_{f} \boldsymbol{k}}^{ \pm}\right|^{2}$. (a,b) Above the Dirac point: $E=0.3 t$. (c,d) Below the Dirac point: $E=-0.3 t$. The green hexagons indicate the first Brillouin zone.

Simulations of the ARPES spectral weight of graphene (not taking into account the polarization term) are shown in Fig. 18, calculated with Eq. 53 and 54. The left column shows constant energy cuts without, the right column with taking into account $M_{\boldsymbol{k}_{f} \boldsymbol{k}}^{ \pm}$. The top row is calculated at $E=0.3 t$, the bottom row at $E=-0.3 t$, 
i.e. above and below the Dirac Point. The archetypical horseshoe pattern is a signature of the interference in between photoelectrons emitted from sites $A$ and $B$, respectively, and nicely mimics the corresponding experimental results (Bostwick et al. , 2007, Hwang et al., 2011; Liu et al., 2011; Mucha-Kruczyński et al. |2008, Puschnig and Lueftner, 2015, Shirley et al., 1995). It is noted however, that a more realistic treatment includes additional plane wave contributions to the final state, which has been shown to restore some of the intensity suppressed by our simple model (Gierz et al., 2011).

\section{H. Matrix elements and spin orbit coupling}

In this section, we aim to describe the basic intensity variations observed in spin polarized two dimensional systems with enhanced spin orbit coupling, like Rashba systems (Ast et al. 2007; Bian et al., 2012, Bihlmayer et al. 2007, Frantzeskakis et al., 2010, 2008; Gierz et al., 2009, Meier et al., 2008, 2009, Nagano et al., 2009; Petersen and Hedegard, 2000; Premper et al. | 2007) and topological insulators (Chen et al. 2009; Hasan and Kane, 2010. Hsieh et al., 2008, Kane, 2008, Kane and Mele, 2005, 2006, König et al., 2007; Xia et al., 2009). Near the $\Gamma$ point and neglecting warping effects, these systems are reasonably well described by a Dirac type effective surface Hamiltonian (Ast and Gierz, 2012, Frantzeskakis and Grioni, 2011; Liu et al. , 2010, 2009, Mao et al. , 2011, Mii et al. 2014; Petersen and Hedegard, 2000, Shanavas et al. 2014, Zhang et al., 2009, 2013)

$$
H=\left(\begin{array}{cc}
\rho k^{2} & \lambda_{R}\left(k_{x}+i k_{y}\right) \\
\lambda_{R}\left(k_{x}-i k_{y}\right) & \rho k^{2}
\end{array}\right),
$$

where $\rho=\hbar^{2} / 2 m$ is the reciprocal band mass, $\lambda_{R}$ is the spin orbit coupling strength and $k^{2}=k_{x}^{2}+k_{y}^{2}$. The basis of this Hamiltonian is formed by states with total angular momentum $J=1 / 2$ and $z$-axis projection $J_{z}= \pm 1 / 2$ : $|\Uparrow\rangle$ and $|\Downarrow\rangle$. A variety of topological insulators such as $\mathrm{Bi}_{2} \mathrm{Se}_{3}, \mathrm{Bi}_{2} \mathrm{Te}_{3}, \mathrm{Sb}_{2} \mathrm{Te}_{3}, \mathrm{Sb}_{2} \mathrm{Se}_{3}$ and Rashba systems such as BiTeBr and BiTeI are members of the family of hexagonal crystal lattices and the basis states close to the Fermi level can be approximated in terms of a symmetry adapted Gutsche and Jahne basis (Bahramy et al. 2011, Jahne and Gutsche, 1967; Lew Yan Voon et al., 1996; Liu et al., 2010, Zhang et al., 2013; Zhu et al., 2014):

$$
|\Uparrow(\Downarrow)\rangle=\alpha\left|s p_{z}, \uparrow(\downarrow)\right\rangle \mp \frac{\beta}{\sqrt{2}}\left[\left|p_{x}, \downarrow(\uparrow)\right\rangle \pm i\left|p_{y}, \downarrow(\uparrow)\right\rangle\right],
$$

where $\left|s p_{z}\right\rangle$ is an appropriate linear combination of orbitals with $s$ and $p_{z}$ character. Both $\alpha$ and $\beta$ are material parameters, reflecting the crystal field splitting as well as the overall spin orbit coupling (Liu et al., 2010 ). Diagonalizing $H$, we find eigenvalues

$$
\varepsilon_{\boldsymbol{k}}^{ \pm}=\rho k^{2} \pm \lambda_{R} k
$$

with eigenvectors

$$
\begin{aligned}
| \pm\rangle & =\frac{1}{\sqrt{2}}\left(|\Uparrow\rangle \pm e^{i \vartheta_{k}}|\downarrow\rangle\right) \\
& =\frac{\alpha}{\sqrt{2}}\left(\left|s p_{z}, \uparrow\right\rangle \pm e^{i \vartheta_{k}}\left|s p_{z}, \downarrow\right\rangle\right) \\
& -\frac{\beta}{2}\left( \pm e^{i \vartheta_{k}}\left|p_{x}, \uparrow\right\rangle-\left|p_{x}, \downarrow\right\rangle\right) \\
& -\frac{i \beta}{2}\left( \pm e^{i \vartheta_{k}}\left|p_{y}, \uparrow\right\rangle+\left|p_{y}, \downarrow\right\rangle\right),
\end{aligned}
$$

where $e^{i \vartheta_{k}}=\left(k_{x}+i k_{y}\right) / k$. Within this minimalistic approach, the matrix elements are obtained by the spinintegrated Fourier transform of this expression

$$
\begin{aligned}
M_{\boldsymbol{k}_{f} \boldsymbol{k}}^{ \pm} & \propto\left\langle\boldsymbol{k}_{f} \mid \pm\right\rangle \\
& =\frac{\alpha}{\sqrt{2}}\left(1 \pm e^{i \vartheta_{\boldsymbol{k}_{f}}}\right)\left\langle\boldsymbol{k}_{f} \mid s p_{z}\right\rangle-\frac{\beta}{2}\left(-1 \pm e^{i \vartheta_{\boldsymbol{k}_{f}}}\right)\left\langle\boldsymbol{k}_{f} \mid p_{x}\right\rangle \\
& -\frac{i \beta}{2}\left(1 \pm e^{i \vartheta_{\boldsymbol{k}_{f}}}\right)\left\langle\boldsymbol{k}_{f} \mid p_{y}\right\rangle
\end{aligned}
$$

The photoemission intensity is given by $\left|M_{\boldsymbol{k}_{f} \boldsymbol{k}}^{ \pm}\right|^{2}$, which after some algebra can be simplified to

$$
\left|M_{\boldsymbol{k}_{f} \boldsymbol{k}}^{ \pm}\right|^{2} \propto \alpha^{2}\left(1 \pm \frac{k_{f x}}{k_{f}}\right)+\frac{\beta^{2}}{2}\left(k_{f}^{2} \pm \frac{\left(3 k_{f y}^{2}-k_{f x}^{2}\right) k_{f x}}{k_{f}}\right)
$$

We identify two major contributions to the photoemission intensity. The first one exhibits $s$ - and $p$-type symmetries and is controlled by $\alpha$, i.e. the fraction of out of plane $s p_{z}$ orbitals contributing to the $J=1 / 2$ manifold. The second term consists of isotropic $s$ - and threefold $f$-type contributions and reflects the in plane orbital contribution $\beta$ to the wave function.

In Fig. 19, we calculate the intensity distribution for a topological insulator with $\rho=0$ at different positions around the Dirac cone depicted in the left column. If we allow only for out of plane orbital components $\beta=$ 0 , we find a half moon shaped intensity pattern, which inverts below the Dirac point. For $\alpha=0$, the intensity pattern becomes threefold, and again, intensity inverts at the crossing point. If we allow for an admixture of in and out of plane orbitals $\alpha=\beta$, the intensity changes from threefold to half moon shaped upon approaching the Dirac point. Indeed, similar intensity patterns have been observed in ARPES experiments on $\mathrm{Bi}_{2} \mathrm{Se}_{3}$ with $\pi$ polarized light (Xie et al., 2014, Zhu et al., 2013).

Fig. 20 shows similar calculations for a hypothetical Rashba system with $\lambda_{R}=0.3 \rho$. Again, $\alpha$ introduces a one- whereas $\beta$ introduces a threefold pattern. Upon 
certain mixing ratios of in and out of plane orbitals, the Rashba states at higher momenta $k_{f}$ can preserve their three-foldness, whereas the states at smaller momenta exhibit the half moon pattern, in apparent contradiction with the six-fold crystal symmetry of the system. Similar intensity patterns have been experimentally observed in BiTeI and $\mathrm{BiAg}_{2}$ (Bentmann, 2012, Crepaldi et al. 2012 Ishizaka et al., 2011).

Let us now assume spin selectivity in the photoemission process, e.g. through a spin filter attached to the photoemission analyzer. Mathematically, this corresponds to a projection of the free electron final state onto one single spin channel, and the photoemission intensity becomes

$$
\begin{aligned}
\left|M_{\boldsymbol{k}_{f} \uparrow \boldsymbol{k}}^{ \pm}\right|^{2} & \propto\left|\left\langle\boldsymbol{k}_{f}, \uparrow \mid \pm\right\rangle\right|^{2} \\
& =\frac{\alpha^{2}}{2}+\frac{\beta^{2}}{4} k_{f}^{2} \pm \frac{\alpha \beta}{\sqrt{2}} \frac{k_{f x}^{2}-k_{f y}^{2}}{k_{f}}
\end{aligned}
$$

and

$$
\begin{aligned}
\left|M_{\boldsymbol{k}_{f} \downarrow \boldsymbol{k}}^{ \pm}\right|^{2} & \propto\left|\left\langle\boldsymbol{k}_{f}, \downarrow \mid \pm\right\rangle\right|^{2} \\
& =\frac{\alpha^{2}}{2}+\frac{\beta^{2}}{4} k_{f}^{2} \mp \frac{\alpha \beta}{\sqrt{2}} \frac{k_{f x}^{2}-k_{f y}^{2}}{k_{f}} .
\end{aligned}
$$

Exemplary calculation results are shown in the far right column of Fig. 19. Interestingly, both $\alpha$ and $\beta$ now individually only contribute isotropic $s$-type spectral weight, whereas the simultaneous contribution of both introduces a two-fold $d$-type signature with a pronounced node along the $k_{x}\left(k_{y}\right)$-axis, which inverts again upon Dirac point crossing. It is interesting to note that such signatures have been observed in spin integrated experiments with $\sigma$-polarized light (Cao et al. , 2013: Jozwiak et al., 2013; Park et al., 2012, Xie et al., 2014, Zhu et al., 2013).

\section{Matrix elements in multilayer systems}

Another interesting set of examples are multilayer systems. Due to a phase difference of the wave functions associated to each layer, a pronounced modulation of the photoemission intensity as a function of $k_{z} \equiv k_{\perp} \propto \sqrt{h \nu}$ has been observed, e.g. for surface states (Louie S. G. et al. 1980) for quantum well states (Mugarza et al. 2000), for multilayer graphene (Ohta et al. |2007) and for bilayer cuprates (Feng et al., 2002$)$ and iridates (Moreschini et al., 2014).

Currently, the iridates draw a lot of attention. Briefly summarized, spin-orbit- coupling and crystal field splitting in these $5 d$ systems order the $t_{2 g}$-bands into a fourfold degenerate and mostly filled $j_{\text {eff }}=3 / 2$ and a narrow two-fold degenerate and nearly half-filled $j_{\text {eff }}=1 / 2$

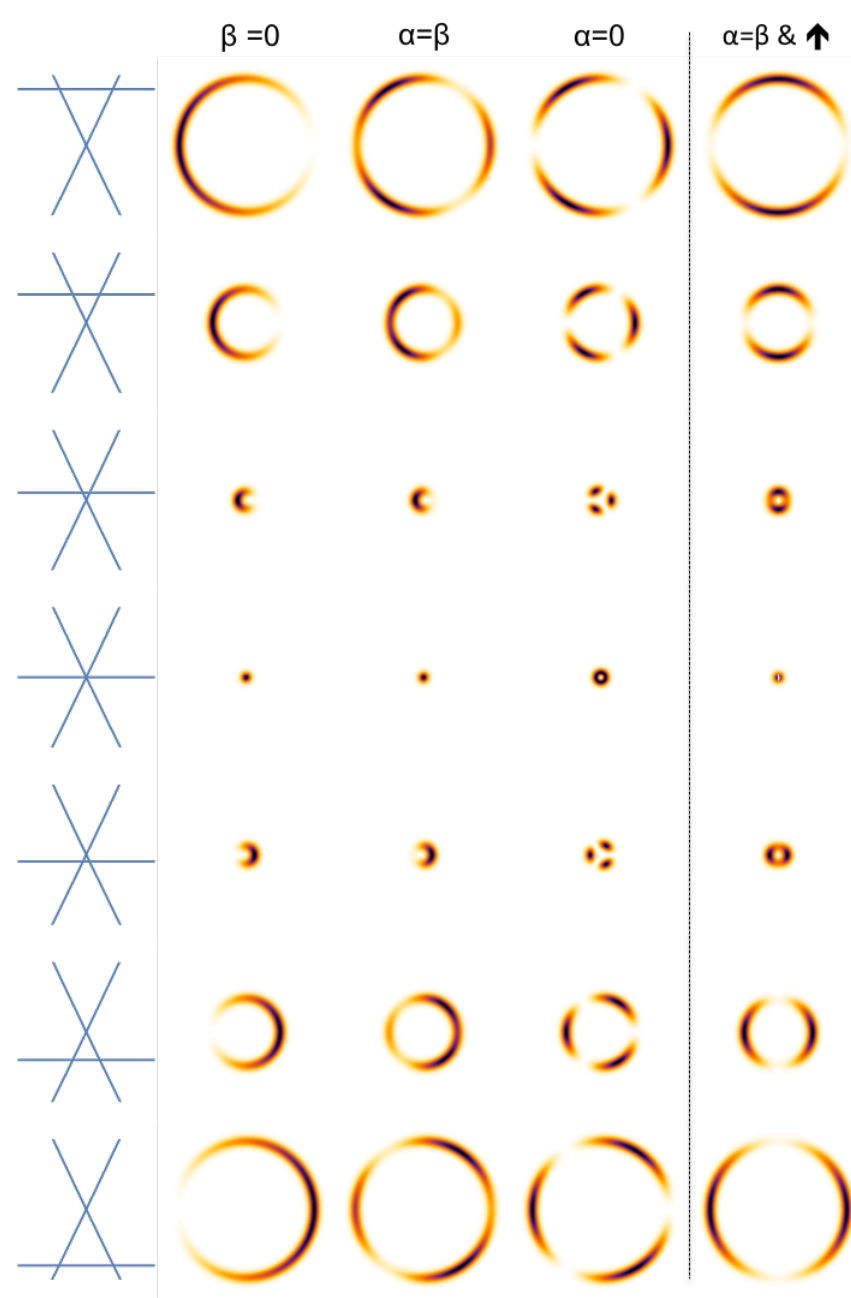

FIG. 19 Stereographic projection of the angular distribution of photoelectrons in a hexagonal topological insulator like $\mathrm{Bi}_{2} \mathrm{Se}_{3}$ calculated for different values of $\alpha$ and $\beta$ at different energy positions within the Dirac cone. The right column shows the final state projection on one single spin component.

band close to the Fermi level. The band-width of the latter is small enough to lift its degeneracy in the presence of correlation $(\sim \mathcal{O}(500) \mathrm{meV})$ and to open a Mott gap (Kim et al. 2008,2009$)$. One of the most prominent examples is $\mathrm{Sr}_{2} \mathrm{IrO}_{4}$. Due to the strong mixing of the orbital degrees in this system, the Wannier orbitals $\left\langle\boldsymbol{r} \mid J_{e f f}, m_{J}\right\rangle$ described by effective angular momenta $J_{\text {eff }}$ and $m_{J}$ are almost isotropic, and so is the ARPES intensity $\left|M_{\boldsymbol{k}_{f} \boldsymbol{k}}^{J_{\text {eff }}, m_{J}}\right|^{2}$ Kim et al. 2009, Moser et al. 2014a).

The bilayer version of $\mathrm{Sr}_{2} \mathrm{IrO}_{4}$ is $\mathrm{Sr}_{3} \mathrm{Ir}_{2} \mathrm{O}_{7}$, shown in Fig. 21 (a). Like the atoms in a hydrogen molecule, the electronic states $\left|\boldsymbol{R}_{\text {layer }}, J_{\text {eff }}, m_{J}\right\rangle$ resulting from each of the layers hybridize, resulting in bonding $(+)$ and antibonding (-) states (Moreschini et al. 2014) 


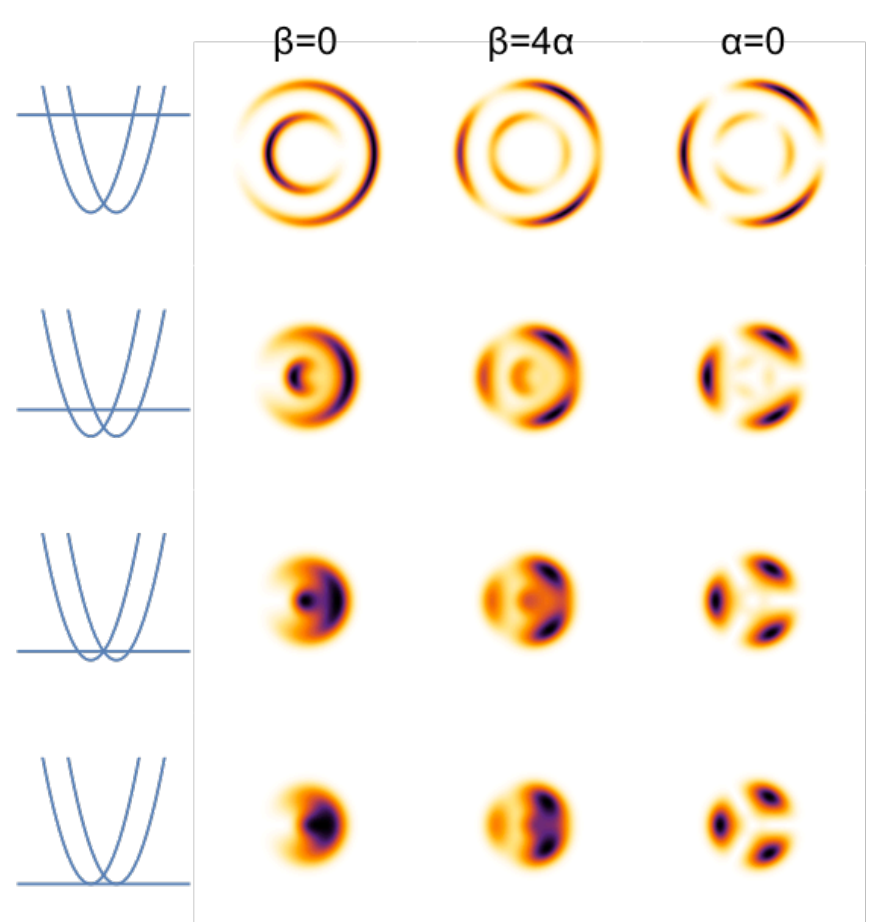

FIG. 20 Stereographic projection of the angular distribution of photoelectrons in a hexagonal topological insulator like BiTeI calculated for different values of $\alpha$ and $\beta$ at different energy positions.

$$
|\mathbf{0}, \pm\rangle=\frac{1}{\sqrt{2}}\left(\left|\boldsymbol{R}_{\mathrm{top}}, J_{\mathrm{eff}}, m_{J}\right\rangle \pm\left|\boldsymbol{R}_{\mathrm{bottom}}, J_{\mathrm{eff}}, m_{J}\right\rangle\right)
$$

whose energy splitting is clearly observed by ARPES (Fig. 21 b) (Moreschini et al., 2014). Due to this hybridization, the photoemission matrix element reads

$$
\begin{aligned}
M_{\boldsymbol{k}_{f} \boldsymbol{k}}^{ \pm} & \propto \frac{1}{\sqrt{2}} M_{\boldsymbol{k}_{f} \boldsymbol{k}}^{J_{\text {eff }}, m_{J}}\left[e^{i \boldsymbol{k}_{f} \cdot \boldsymbol{R}_{\mathrm{top}}} \pm e^{i \boldsymbol{k}_{f} \cdot \boldsymbol{R}_{\mathrm{bottom}}}\right] \\
& =\frac{1}{\sqrt{2}} M_{\boldsymbol{k}_{f} \boldsymbol{k}}^{J_{\text {eff }}, m_{J}}\left[e^{-i \frac{d}{2} k_{z}} \pm e^{i \frac{d}{2} k_{z}}\right] \\
& =\frac{2}{\sqrt{2}} M_{\boldsymbol{k}_{f} \boldsymbol{k}}^{J_{\text {eff }}, m_{J}} \times\left\{\begin{array}{c}
\cos \left(\frac{d}{2} k_{z}\right) \\
i \sin \left(\frac{d}{2} k_{z}\right)
\end{array},\right.
\end{aligned}
$$

Fig. 21 (c) shows the $k_{z}$-dependence of the ARPES signal at the $\Gamma$-point. As the model predicts, the spectral weight oscillates between the bonding $(+)$ and antibonding $(-)$ states with periodicity $d \sim c / 5$.

\section{J. Matrix elements in doped oxides}

Let us now test this formalism for systems of much higher complexity. A particularly interesting class of materials are the $d^{0}$ transition metal oxides, some of which
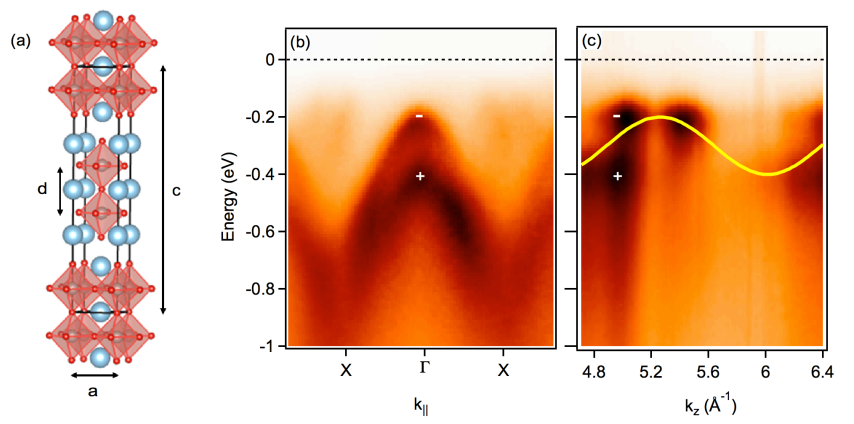

FIG. 21 (a) Structural model of $\mathrm{Sr}_{3} \mathrm{Ir}_{2} \mathrm{O}_{7}$. (b) ARPES intensity map along $X \Gamma X$ showing the energy splitting in between bonding $(+)$ and anti bonding $(-)$ states. (c) Intensity oscillation of bonding and antibonding states as a function of $k_{z}$. The model function $2 \sin ^{2}\left(\frac{d}{2} k_{z}\right)=1-\cos \left(d k_{z}\right)$ is superimposed in yellow. For a more detailed discussion on this topic, the reader is referred to Moreschini et al. 2014.

can be doped and become metallic. Prominent examples like $\mathrm{SrTiO}_{3}$ (Meevasana et al. , 2011, Santander-Syro et al., 2011), $\mathrm{KTaO}_{3}$ (King et al. | 2012) or $\mathrm{TiO}_{2}$ anatase (Moser et al., 2013) form metallic surface states upon electron doping. Interestingly, the conduction states in these systems retain their orbital order. In anatase for example, the conduction band states are of almost pure Ti $3 d_{x y}$ orbital character (Asahi et al., 2000) and the initial state Wannier orbital can be approximated as

$$
\left|d_{x y}\right\rangle=\frac{1}{i \sqrt{2}}(|2,2\rangle-|2,-2\rangle) .
$$

Due to the nodes of this orbital along the $x z$ and $y z$ plane, we expect the photoemission signal to vanish exactly along $k_{x}=0$ and $k_{y}=0$. In the real experiment however, the sample is typically rotated by an angle $\beta$ to yield the $k_{y}$ dimension, which softens this constraint (cf. Fig. 3). According to Eq. 48, the matrix element becomes 


$$
\begin{aligned}
M_{\boldsymbol{k}_{f}}^{d_{x y}}(\theta, \beta, \phi) & \propto \frac{1}{i \sqrt{2}} \sum_{m^{\prime}=-2}^{2}\left(D_{m^{\prime} 2}^{2}(\theta, \beta, \phi) M_{\boldsymbol{k}_{f}}^{2 m^{\prime}}-D_{m^{\prime}-2}^{2}(\theta, \beta, \phi) M_{\boldsymbol{k}_{f}}^{2 m^{\prime}}\right) \\
& =\frac{1}{i \sqrt{2}} \sum_{m^{\prime}=-2}^{2} M_{\boldsymbol{k}_{f}}^{2 m^{\prime}}\left(D_{m^{\prime} 2}^{2}(\theta, \beta, \phi)-D_{m^{\prime}-2}^{2}(\theta, \beta, \phi)\right) \\
& =\frac{1}{i} \sum_{d^{\prime}} M_{\boldsymbol{k}_{f}}^{d^{\prime}} D_{d^{\prime} d_{x y}}(\theta, \beta, \phi),
\end{aligned}
$$

where $d^{\prime}$ runs over the whole set $x y, x^{2}-y^{2}, x z, y z$ and $z^{2}$ of $d$-orbitals and the coefficients $D_{d^{\prime} d_{x y}}$ are given in Sec. VII.
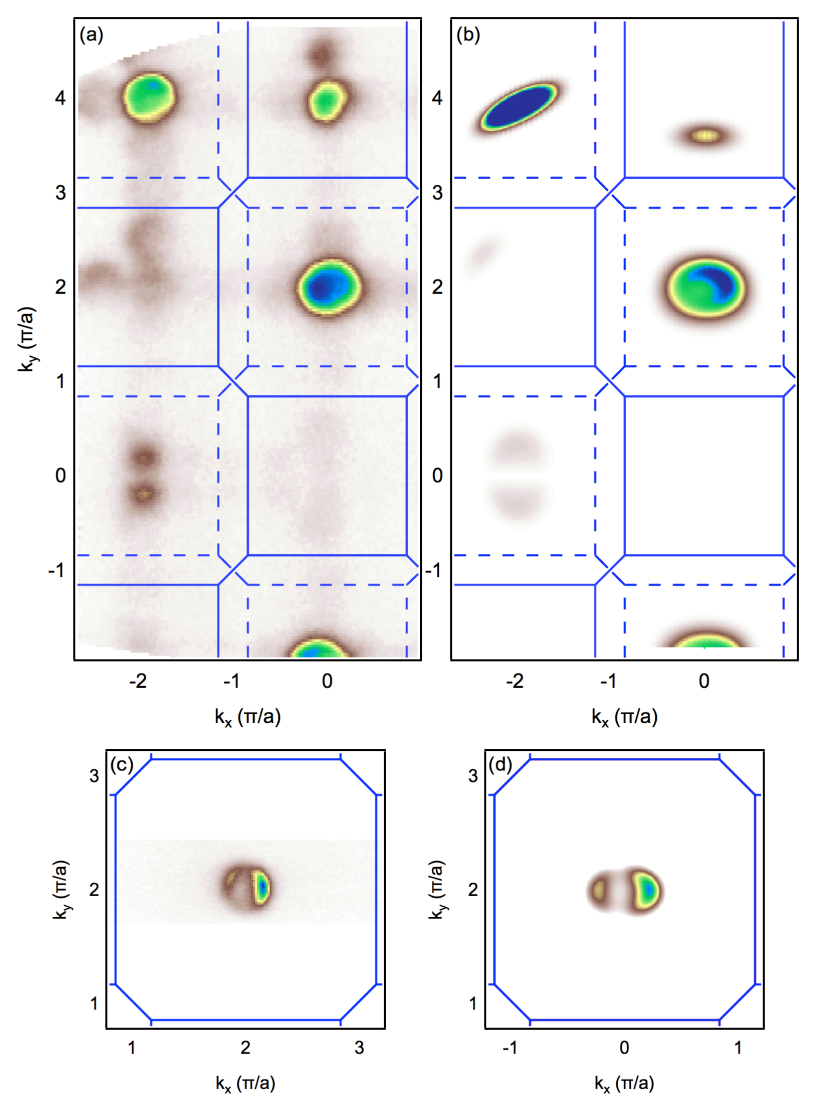

FIG. 22 (a) Measured and (b) simulated ARPES Fermi surfaces of anatase at $85 \mathrm{eV}$ photon energy. Blue lines indicate projections of the $3 \mathrm{D}$ Brillouin zone. Along $k_{y}=0$, spectral weight is completely suppressed but recovers for $k_{y} \neq 0$ due to the sample rotation. (c) Close up measurement of the electron pocket at $\left(k_{x}, k_{y}\right)=(0,2 \pi / a)$ compared to the simulation (d) with $\theta=0.5^{\circ}$. For more details on experiment and interpretation, the reader is referred to Moser et al. 2013.

To simulate the spectral function of the electron pocket, we use a tight binding dispersion $\varepsilon_{\boldsymbol{k}}=$ $-8 \cos \left(a k_{x} / 2\right) \cos \left(a k_{y} / 2\right) \cos \left(a k_{z} / 2\right)+\mu$ adapted to the periodicity to the bct 2 lattice of anatase. The chemical potential $\mu=7.7 \mathrm{eV}$ was fixed to mimic the experimen- tal observation. The ARPES intensity is calculated from Eq. 54, approximating the spectral function by a Gaus$\operatorname{sian}(\mathrm{Eq} \cdot 53$ ).

A Fermi surface of anatase measured with $85 \mathrm{eV}$ photons is given in Fig. 22 (a) and compared directly to the calculation results in (b). For a detailled discussion of experiment and interpretation, the reader is referred to Moser et al. 2013. The suppression of spectral weight at normal emission and along $k_{y}=0$ is nicely reproduced. Close to $\left(k_{x}, k_{y}\right)=(0,2 \pi / a)$, spectral weight reappears as result of the $\beta$-rotation of the sample in this experiment.

A close up of the electron pocket of the Brillouin zone centered at $(0,2 \pi / a)$ measured with $\theta \sim 0$ is shown in Fig. 22 (c). We now clearly see residual suppression of the electron pocket along $k_{x}=0$. The intensity distribution can be well reproduced taking into account a small misalignment of $\theta=0.5^{\circ}$ in the calculation of Fig. 22 (d).

The discrepancies in between calculation and measurement towards large $k_{y}$ can to some extent be related to the simplistic modeling of the initial state and the dispersion relation. Nevertheless, it has to be noted that in complex oxides, the validity of the free electron final state approximation is at its limits and lifetime effects in both the initial and final state will become more important.

\section{K. Matrix elements in cuprates}

Finally, we apply this intensity formalism to the twodimensional cuprates. The important structural unit in the high-temperature cuprate superconductors is the ubiquitous two dimensional $\mathrm{CuO}_{2}$ plane, with $\mathrm{Cu} 3 d_{x^{2}-y^{2}}$ and $\mathrm{O} 2 p_{x, y}$ orbitals forming the backbone of its low energy electronic structure (Eskes et al., 1990; Ghijsen et al., 1988, Zaanen et al., 1985). In the undoped parent compounds, copper has a nominal valence of $\mathrm{Cu}^{2+}$ with a $3 d^{9}$ electron configuration. As a consequence of the tetrahedral crystal field, the single hole occupies the $\mathrm{Cu}$ $3 d_{x^{2}-y^{2}}$ orbital.

Doping the system or performing photoemission introduces additional holes (Damascelli et al., 2003). Due to the large on site Coulomb interaction of the $3 d$ orbitals, these holes preferentially occupy the ligand O $2 p$ orbitals resulting in a $d^{9} \underline{L}$ configuration (Eskes et al., 1990; Za- 
anen et al., 1985). Through superexchange interaction, the $\mathrm{Cu} 3 d$ hole and the $\mathrm{O} 2 p$ hole form a quasi bound state of singlet character and ${ }^{1} A_{1}$ symmetry - which is energetically preferred to the ${ }^{3} B_{1}$ Hund's rule ground state and propagates as an effective one particle entity the Zhang Rice Singlet (ZRS) (Zhang and Rice, 1988). Formally, this state is constructed from the $\mathrm{Cu} d_{x^{2}-y^{2}}$ orbital and a linear combination of the four surrounding oxygen $p_{x / y}$-orbitals with equivalently $x^{2}-y^{2}$ symmetry
(Eskes et al. 1990). Consequently, the angular part of the emitting orbital can be written as

$$
\left|d_{x^{2}-y^{2}}\right\rangle=\frac{1}{\sqrt{2}}(|2,2\rangle+|2,-2\rangle) .
$$

According to Eq. 48 the matrix element under rotation becomes

$$
\begin{aligned}
M_{\boldsymbol{k}_{f}}^{d_{x^{2}-y^{2}}}(\theta, \beta, \phi) & \propto \frac{1}{\sqrt{2}} \sum_{m^{\prime}=-2}^{2}\left(D_{m^{\prime} 2}^{2}(\theta, \beta, \phi) M_{\boldsymbol{k}_{f}}^{2 m^{\prime}}+D_{m^{\prime}-2}^{2}(\theta, \beta, \phi) M_{\boldsymbol{k}_{f}}^{2 m^{\prime}}\right) \\
& =\frac{1}{\sqrt{2}} \sum_{m^{\prime}=-2}^{2} M_{\boldsymbol{k}_{f}}^{2 m^{\prime}}\left(D_{m^{\prime} 2}^{2}(\theta, \beta, \phi)+D_{m^{\prime}-2}^{2}(\theta, \beta, \phi)\right) \\
& =\sum_{d^{\prime}} M_{\boldsymbol{k}_{f}}^{d^{\prime}} D_{d^{\prime} d_{x^{2}-y^{2}}}(\theta, \beta, \phi) .
\end{aligned}
$$

We illustrate this result with ARPES data obtained on tetragonal $\mathrm{CuO}$ (Moser et al. . 2014b). To simulate the spectral function of the ZRS, we use a mean field dispersion of the extended $t$-J-model $\varepsilon_{\boldsymbol{k}}=$ $-4 t^{\prime} \cos \left(a k_{x}\right) \cos \left(a k_{y}\right)-2 t^{\prime \prime}\left(\cos \left(2 a k_{x}\right)+\cos \left(2 a k_{y}\right)\right)-$ $J\left(\cos \left(a k_{x}\right)+\cos \left(a k_{y}\right)\right)^{2}-4 t_{d} \cos \left(a k_{x} / 2\right) \cos \left(a k_{y} / 2\right)$ with $t^{\prime}=-40 \mathrm{meV}, t^{\prime \prime}=12 \mathrm{meV}, J=120 \mathrm{meV}$ and $t_{d}=-40 \mathrm{meV}$ which fits our quasi particle dispersion well. The ARPES intensity is again calculated from Eq. 54, approximating the spectral function by a Gaus$\operatorname{sian}(\mathrm{Eq} .53)$.

Fig. 23 illustrates the effect of the matrix elements on the ZRS band. Top (1) and bottom (2) row show $k_{x} k_{y}$ intensity maps around $k_{x}=0 \AA^{-1}\left(\theta=0^{\circ}\right)$ and $k_{x}=-3 \AA^{-1}\left(\theta=36^{\circ}\right)$, respectively. Fig. 23 (a) displays the experimental result at the top of the ZRS band at $\sim-2.3 \mathrm{eV}$. Panel (b) mimics the spectral function without matrix elements. The same intensity maps, multiplied by the angle-dependent modulus square of the matrix elements of the $d_{x^{2}-y^{2}}$ orbital, are shown in panel (c). One observes a general reduction of intensity around normal emission $\left(k_{x}=k_{y}=0\right)$. Intensity is also suppressed along the diagonals $k_{x}= \pm k_{y}$ - but not along the diagonals of a generic Brillouin zone - consistent with the experimental observations. Even more than in the $d^{0}$ oxides, many particle and lifetime effects have to be considered in the cuprate compounds. This brief assessment of the photoemission intensity such has to be taken as a crude thumb-rule estimate of the physical reality.

\section{CONCLUDING REMARKS}

Throughout this work, we developed a consistent and intuitive formalism to describe ARPES "matrix element effects" in their most simple form. In the free electron final state approximation, the ARPES intensity distribution was shown to be essentially determined by the Fourier transform $\left\langle\boldsymbol{k}_{f} \mid \mathbf{0}, n\right\rangle$ of the local Wannier orbital associated to the emitting Bloch band, which reflects the overall orbital symmetry of this state. An additional polarization term $\boldsymbol{\epsilon} \cdot\left(i \boldsymbol{k}_{f}-\frac{1}{\lambda} \boldsymbol{e}_{\perp}\right)$ further enforces the dipole selection rules. Despite the simplicity of this term, it gives rise to surprising geometric effects, one of them being $p$-type circular dichroism as direct consequence of the handedness of the ARPES experiment. Even if this formalism inherently reflects a one particle picture, it still seems to be useful for interacting systems where all spectral contributions corresponding to one distinct initial state can be identified (cf. Eq. 8). The global validity of the latter hypothesis however needs to be carefully tested and in most cases, an approach based on Dyson orbitals might be the better method of choice (Dauth et al., 2014). Nevertheless, the free electron final state approximation satisfactorily describes a number of molecular and solid state systems of varying complexity.

Upon discussing some showcases however, it became very clear that this simple approach also suffers some deficits. One point is the questionable validity of the free electron final state approximation pointed out recently by Bradshaw and Woodruff (2015), which neglects the spherical-wave nature of the initial emission and elastic scattering of the outgoing photoelectron (Feder et al. 1983: Osterwalder et al. 1996). The introduction of the phenomenological final state penetration depth $\lambda$ gives 

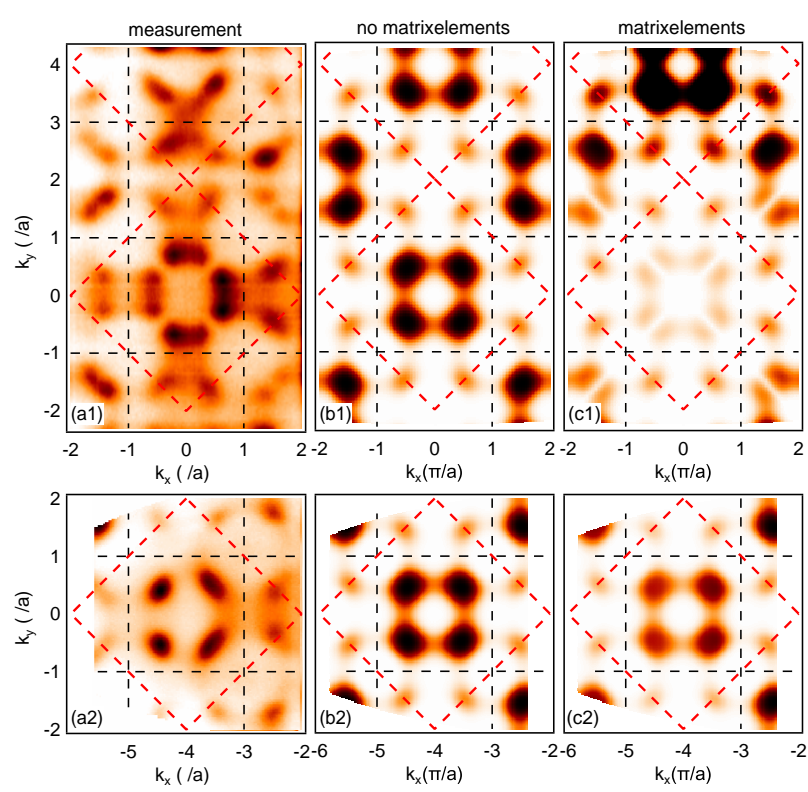

FIG. 23 ARPES constant energy maps at the top of the ZRS band at $-2.3 \mathrm{eV}$ measured with $120 \mathrm{eV}$ photons. (a) ARPES measurement. (b) Simulated spectral function without matrix elements. (c) Spectral function weighted by matrix elements of the $\mathrm{Cu} d_{x^{2}-y^{2}}$ orbital $\left|\mathbf{0}, d_{x^{2}-y^{2}}\right\rangle$. The top row (1) shows results for a sample pointing towards the detector $(\theta=0)$, the bottom row (2) for a sample rotated by $\theta=36^{\circ}$. The intensity is clearly suppressed along the $k_{x}= \pm k_{y}$ axis as result of the node in the emitting orbital. For more details on experiment and interpretation, the reader is referred to Moser et al. 2014b.

good qualitative insights to the influence of the sample surface, like the out of plane "pseudo-momentum conservation" or the experimental handedness, the microscopic descriptive power of this quantity is however rather questionable.

This becomes most obvious in noticing that this description models the surface by an exponential cutoff of a Wannier orbital representative of the material's bulk. A more reasonable approach of course requires a detailed microscopic modeling of the sample's surface, and of the photoelectron's final state. A still simplistic but more accurate approach might be to consider more than just one plane wave component of the final state in Eq. 16 . especially at low photon energies. This has been shown to give good results in systems like graphene, where a finite but manageable number of plane wave components to the final state results in a more realistic description of ARPES spectral weight (Gierz et al., 2011).

Another problem lies in the action of the polarization term $\boldsymbol{\epsilon} \cdot\left(i \boldsymbol{k}_{f}-\frac{1}{\lambda} \boldsymbol{e}_{\perp}\right)$, which in this approach shows no coupling effect to the initial state. Namely, it is incapable of selecting states of distinct orbital symmetry, and therefore incapable in predicting any form of intrinsic circular dichroism. Additionally, this term is identical to zero for all photoelectrons probed at normal emission with $\sigma$-polarized light - describing the experimental trend of overall lower intensities - but in definite contrast to the selective power of $\sigma$-polarization in a variety of materials. Again, taking into account more but one plane wave components to the final state could potentially resolve this problem. In many cases however, a full one step approach involving relativistic time-reversed low energy electron diffraction final states and a proper many body description is probably inevitable (Braun, 1996, Braun and Donath, 2004, Minár, 2014; Minár et al., 2011).

\section{ACKNOWLEDGEMENTS}

I would like to thank Hendrik Bentmann, Mona Berciu, Silke Biermann, Aaron Bostwick, Ashish Chainani, Young Jun Chang, Federico Cilento, Ralph Claessen, Alberto Crepaldi, Chuck Fadley, Sara Fatale, Emmanouil Frantzeskakis, Martin Graus, Michael Greif, Marco Grioni, Yoshihisa Harada, Karsten Horn, Jens Johannsen, Beomyoung Kim, Roland Koch, Peter Krüger, Mathieu Leclerc, José Lorenzana, Chris Jozwiak, Peter Lutz, Henriette Maaß, Daniel Malterre, Giulia Manzoni, Andrea Marini, Luca Moreschini, Ngoc Linh Nguyen, Yusuke Nomura, Luca Perfetti, Fulvio Parmigiani, Friedel Reinert, Tobias Rödel, Victor Rogalev, Eli Rotenberg, Andres Santander-Syro, Achim Schöll, Daniel Schwarz, Denys Sutter, Cédric Tournier-Colletta, Søren Ulstrup, Guillaume Vasseur and HyangKeun Yoo for many helpful discussions and lots of constructive feedback on this topic. This research was supported by the Swiss National Science Foundation, in particular through Grant No. P2ELP2-155357. The Advanced Light Source is supported by the Director, Office of Science, Office of Basic Energy Sciences, of the U.S. Department of Energy under Contract No. DE-AC02-05CH11231.

\section{APPENDIX: ROTATION OF ATOMIC ORBITALS}

Rotations in 3D space are expressed in terms of an orthogonal $3 \times 3$ matrix $\mathcal{R}$. Under such a rotation, the spherical harmonics $|l m\rangle$ transform according to the coefficients $D_{m^{\prime} m}^{l}=\left\langle l m^{\prime}|U| l m\right\rangle$ of the Wigner D-matrix, where $U$ is a unitary transformation rotating the atomic orbitals according to $\mathcal{R}$.

Typically, the Wigner D-Matrix is expressed in terms of Euler angles in the $z-x-z$ convention, namely a first rotation $\alpha_{z_{0}}$ around the $z$-axis, a second rotation $\alpha_{x}$ around the $x$-axis and a third rotation $\alpha_{z_{1}}$ again around the $z$ axis, where the rotation axes are referenced to the rotating object. The Wigner D-coefficients are then given by

$$
D_{m^{\prime} m}^{l}\left(\alpha_{z_{0}}, \alpha_{x}, \alpha_{z_{1}}\right)=e^{-i m^{\prime} \alpha_{z_{1}}} d_{m^{\prime} m}^{l}\left(\alpha_{x}\right) e^{-i m \alpha_{z_{0}}},
$$


where

$$
d_{m^{\prime} m}^{l}\left(\alpha_{x}\right)=\left\langle l m^{\prime}\left|e^{-i \alpha_{x} L_{x}}\right| l m\right\rangle,
$$

and $L_{x}$ is the $x$-component of the angular momentum operator.

However, great care has to be taken to express $\alpha_{z_{0}}$, $\alpha_{x}$ and $\alpha_{z_{1}}$ in terms of an arbitrary rotation $\mathcal{R}$ as such a factorization is in general not unique (Eberly, 2008). Therefore, we give the Wigner D-coefficients directly for the $s, p, d$ and $f$ orbitals in terms of an arbitrary rotation matrix $\mathcal{R}$ (Stewart, 2007), which has to be constructed according to the rotation properties of the sample manip- ulator of the individual experiment. In the particular example of the $\mu$ ARPES setup of the MAESTRO beamline at the Advanced Light Source, the sample manipulator turns axially around the $x$-axis by an angle $\theta$ as defined in Fig. 3 A rotation stage which is mounted on the manipulator allows for sample tilts $\beta$ around the $y$-axis and finally for azimuthal rotations $\phi$ around the $z$-axis. To obtain the full $k_{x} k_{y}$-space information by ARPES, the sample tilt $\beta$ is typically scanned at fixed angles $\theta$ and $\phi$. Accordingly to this $x-y$ - $z$-scheme, the rotation matrix in this particular experiment is constructed as

$$
\begin{aligned}
& \mathcal{R}=\left(\begin{array}{lll}
a & b & c \\
d & e & f \\
g & h & o
\end{array}\right)=\left(\begin{array}{ccc}
\cos \phi & -\sin \phi & 0 \\
\sin \phi & \cos \phi & 0 \\
0 & 0 & 1
\end{array}\right) \cdot\left(\begin{array}{ccc}
\cos \beta & 0 & -\sin \beta \\
0 & 1 & 0 \\
\sin \beta & 0 & \cos \beta
\end{array}\right) \cdot\left(\begin{array}{ccc}
1 & 0 & 0 \\
0 & \cos \theta & -\sin \theta \\
0 & \sin \theta & \cos \theta
\end{array}\right) \\
& =\left(\begin{array}{ccc}
\cos \beta \cos \phi & -\cos \phi \sin \beta \sin \theta-\cos \theta \sin \phi & \sin \theta \sin \phi-\cos \theta \cos \phi \sin \beta \\
\cos \beta \sin \phi & \cos \theta \cos \phi-\sin \beta \sin \theta \sin \phi & -\cos \phi \sin \theta-\cos \theta \sin \beta \sin \phi \\
\sin \beta & \cos \beta \sin \theta & \cos \beta \cos \theta
\end{array}\right) \text {. } \\
& \begin{array}{l|l}
D_{s^{\prime} s}^{0} & s \\
\hline s^{\prime} & 1
\end{array} \\
& \begin{array}{c|ccc}
D_{p^{\prime} p}^{1} & p_{x} & p_{y} & p_{z} \\
\hline p_{x}^{\prime} & a & b & c \\
p_{y}^{\prime} & d & e & f \\
p_{z}^{\prime} & g & h & o
\end{array}
\end{aligned}
$$

\begin{tabular}{|c|c|c|}
\hline$D_{f^{\prime} f}^{3}$ & $f_{z\left(x^{2}-y^{2}\right)}$ & $f_{x z^{2}}$ \\
\hline$f_{x\left(x^{2}-3 y^{2}\right)}^{\prime}$ & $\frac{1}{2} \sqrt{\frac{3}{2}}\left(c\left(a^{2}-b^{2}-d^{2}+e^{2}\right)-2 a d f+2 b e f\right)$ & $-\frac{1}{4} \sqrt{\frac{3}{5}}\left(a^{3}+\left(b^{2}-4 c^{2}-3 d^{2}-e^{2}+4 f^{2}\right) a-2 b d e+8 c d f\right)$ \\
\hline $\begin{array}{c}f_{y\left(3 x^{2}-y^{2}\right)}^{\prime} \\
f_{x y z}^{\prime}\end{array}$ & $\begin{array}{c}\frac{1}{2} \sqrt{\frac{3}{2}}\left(f a^{2}+2 c d a-2 b c e-b^{2} f+\left(e^{2}-d^{2}\right) f\right) \\
c d g+a f g-c e h-b f h+a d o-b e o\end{array}$ & $\begin{array}{c}-\frac{1}{4} \sqrt{\frac{3}{5}}\left(3 d a^{2}+2(b e-4 c f) a-d\left(-b^{2}+4 c^{2}+d^{2}+e^{2}-4 f^{2}\right)\right) \\
-\frac{3 a d g+b e g-4 c f g+b d h+a e h-4(c d+a f) o}{3-a n}\end{array}$ \\
\hline$f_{z\left(x^{2}-y^{2}\right)}^{\prime}$ & $\frac{1}{2}\left(o a^{2}+2 c g a-2 d f g-2 b c h+2 e f h-b^{2} o-d^{2} o+e^{2} o\right)$ & $-\frac{3 g a^{2}+2 b h a-8 c o a+b^{2} g-4 c^{2} g-3 d^{2} g-e^{2} g+4 f^{2} g-2 d e h+8 d f o}{2 \sqrt{10}}$ \\
\hline$f_{x z^{2}}^{\prime}$ & $-\frac{3 c a^{2}+2(d f-4 g o) a-3 b^{2} c+c\left(d^{2}-e^{2}-4 g^{2}+4 h^{2}\right)+b(8 h o-2 e f)}{2 a^{2}\left(2 c d a-4 f g^{2}+4 f h^{2}-2 b c e-b^{2} f+3 d^{2} f-3 e^{2} f-8 d q o+8 e h o\right.}$ & $\frac{1}{20}\left(3 a^{3}+\left(3 b^{2}-12 c^{2}+3 d^{2}+e^{2}-4 f^{2}-12 g^{2}-4 h^{2}+16 o^{2}\right) a+2 b d e-8 c d f-8 b g h+32 c g o\right)$ \\
\hline $\begin{array}{l}f_{y z^{2}} \\
f_{z^{3}}^{\prime}\end{array}$ & $-\frac{1}{2} \sqrt{\frac{3}{5}}\left(o a^{2}+2 c g a+2 d f g-2(b c+e f) h+d^{2} o-\left(b^{2}+e^{2}+2 g^{2}-2 h^{2}\right) o\right)$ & $\begin{array}{l}\frac{1}{20}\left(3 d^{3}+3 a^{2} d+b^{2} d-4 c^{2} d+3 e^{2} d-12 f^{2} d-12 g^{2} d-4 h^{2} d+16 o^{2} d+2 a b e-8 a c f-8 e g h+32 f g o\right) \\
\frac{1}{10} \sqrt{\frac{3}{2}}\left(-2 g^{3}+3 a^{2} g+b^{2} g-4 c^{2} g+3 d^{2} g+e^{2} g-4 f^{2} g-2 h^{2} g+8 o^{2} g+2 a b h+2 d e h-8 a c o-8 d f o\right)\end{array}$ \\
\hline
\end{tabular}

\begin{tabular}{c|ccccc}
$D_{d^{\prime} d}^{2}$ & $d_{x y}$ & $d_{x^{2}-y^{2}}$ & $d_{x z}$ & $d_{y z}$ & $d_{z^{2}}$ \\
\hline$d_{x y}^{\prime}$ & $b d+a e$ & $a d-b e$ & $c d+a f$ & $c e+b f$ & $-\frac{a d+b e-2 c f}{\sqrt{3}}$ \\
$d_{x^{2}-y^{2}}^{\prime}$ & $a b-d e$ & $\frac{1}{2}\left(a^{2}-b^{2}-d^{2}+e^{2}\right)$ & $a c-d f$ & $b c-e f$ & $\frac{-a^{2}-b^{2}+2 c^{2}+d^{2}+e^{2}-2 f^{2}}{2 \sqrt{3}}$ \\
$d_{x z}^{\prime}$ & $b g+a h$ & $a g-b h$ & $c g+a o$ & $c h+b o$ & $-\frac{a g+b h-2 c o}{\sqrt{3}}$ \\
$d_{y z}^{\prime}$ & $e g+d h$ & $d g-e h$ & $f g+d o$ & $f h+e o$ & $-\frac{d g+e h-2 f o}{\sqrt{3}}$ \\
$d_{z^{2}}^{\prime}$ & $-\frac{a b+d e-2 g h}{\sqrt{3}}$ & $-\frac{a^{2}-b^{2}+d^{2}-e^{2}-2 g^{2}+2 h^{2}}{2 \sqrt{3}}$ & $-\frac{a c+d f-2 g o}{\sqrt{3}}$ & $-\frac{b c+e f-2 h o}{\sqrt{3}}$ & $\frac{1}{6}\left(a^{2}+b^{2}-2 c^{2}+d^{2}+e^{2}-2\left(f^{2}+g^{2}+h^{2}-2 o^{2}\right)\right)$
\end{tabular}

\begin{tabular}{c|ccc}
$D_{f^{\prime} f}^{3}$ & $f_{x\left(x^{2}-3 y^{2}\right)}$ & $f_{y\left(3 x^{2}-y^{2}\right)}$ & $f_{x y z}$ \\
\hline$f_{x\left(x^{2}-3 y^{2}\right)}^{\prime}$ & $\frac{1}{4}\left(a^{3}-3\left(b^{2}+d^{2}-e^{2}\right) a+6 b d e\right)$ & $\frac{1}{4}\left(3 b a^{2}-6 d e a-b\left(b^{2}+3 d^{2}-3 e^{2}\right)\right)$ & $-\sqrt{\frac{3}{2}}(-a b c+d e c+b d f+a e f)$ \\
$f_{y\left(3 x^{2}-y^{2}\right)}^{\prime}$ & $\frac{1}{4}\left(3 d a^{2}-6 b e a-d\left(3 b^{2}+d^{2}-3 e^{2}\right)\right)$ & $\frac{1}{4}\left(e^{3}+3 a^{2} e-3 b^{2} e-3 d^{2} e+6 a b d\right)$ & $\sqrt{\frac{3}{2}}(b c d-e f d+a c e+a b f)$ \\
$f_{x y z}^{\prime}$ & $-\sqrt{\frac{3}{2}}(-a d g+b e g+b d h+a e h)$ & $\sqrt{\frac{3}{2}}(b d g+a e g+a d h-b e h)$ & $c e g+b f g+c d h+a f h+b d o+a e o$ \\
$f_{z\left(x^{2}-y^{2}\right)}^{\prime}$ & $\sqrt{2} \sqrt{\frac{3}{2}}\left(\left(a^{2}-b^{2}-d^{2}+e^{2}\right) g-2 a b h+2 d e h\right)$ & $\frac{1}{2} \sqrt{\frac{3}{2}}\left(h a^{2}+2 b g a-2 d e g-d^{2} h+\left(e^{2}-b^{2}\right) h\right)$ & $b c g-e f g+a c h-d f h+a b o-d e o$ \\
$f_{x z^{2}}^{\prime}$ & $-\frac{1}{4} \sqrt{\frac{3}{5}}\left(a^{3}+\left(-3 b^{2}+d^{2}-e^{2}-4 g^{2}+4 h^{2}\right) a-2 b d e+8 b g h\right)$ & $-\frac{1}{4} \sqrt{\frac{3}{5}}\left(3 b a^{2}+2(d e-4 g h) a-b\left(b^{2}-d^{2}+e^{2}+4 g^{2}-4 h^{2}\right)\right)$ & $-\frac{c d e+b d f-4 c g h-4 b g o+a(3 b c+e f-4 h o)}{\sqrt{10}}$ \\
$f_{y z^{2}}^{\prime}$ & $-\frac{1}{4} \sqrt{\frac{3}{5}}\left(d^{3}+a^{2} d-b^{2} d-3 e^{2} d-4 g^{2} d+4 h^{2} d-2 a b e+8 e g h\right)$ & $\frac{1}{4} \sqrt{\frac{3}{5}}\left(-e a^{2}-2 b d a-4 e h^{2}+e\left(b^{2}-3 d^{2}+e^{2}+4 g^{2}\right)+8 d g h\right)$ & $-\frac{b c d+3 e f d-4 h o d+a c e+a b f-4 f g h-4 e g o}{\sqrt{10}}$ \\
$f_{z^{3}}^{\prime}$ & $-\frac{-2 g^{3}+3 a^{2} g-3 b^{2} g+3 d^{2} g-3 e^{2} g+6 h^{2} g-6 a b h-6 d e h}{2 \sqrt{10}}$ & $-\frac{2 h^{3}+3 a^{2} h+3 d^{2} h-3\left(b^{2}+e^{2}+2 g^{2}\right) h+6 a b g+6 d e g}{2 \sqrt{10}}$ & $-\sqrt{\frac{3}{5}}(b c g+e f g-2 h o g+a c h+d f h+a b o+d e o)$
\end{tabular}




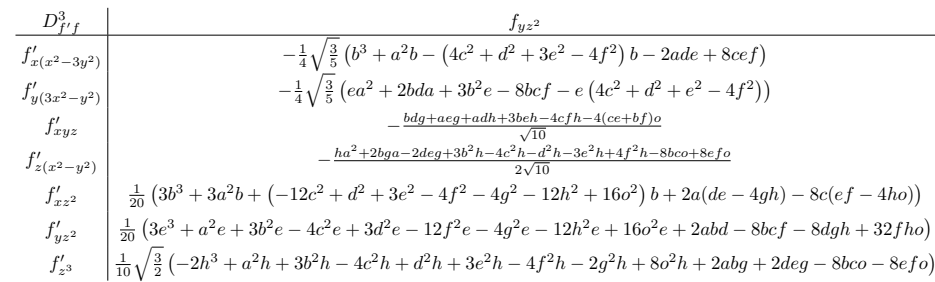

\section{REFERENCES}

Anderson, J., G. J. Lapeyre, and R. J. Smith (1978), Physical Review B 17 (6), 2436.

Arfken, G. B., H. J. Weber, and F. E. Harris (2012), Mathematical methods for physicists, 7th ed. (Elsevier Academic Press).

Armitage, N. P., D. H. Lu, C. Kim, A. Damascelli, K. M. Shen, F. Ronning, D. L. Feng, P. Bogdanov, X. J. Zhou, W. L. Yang, Z. Hussain, P. K. Mang, N. Kaneko, M. Greven, Y. Onose, Y. Taguchi, Y. Tokura, and Z.-X. Shen (2003), Physical Review B 68 (6), 064517

Arpiainen, V., V. Zalobotnyy, A. A. Kordyuk, S. V. Borisenko, and M. Lindroos (2008), Physical Review B 77 (2), 024520.

Arrälä, M., J. Nieminen, J. Braun, H. Ebert, and M. Lindroos (2013), Physical Review B 88 (19), 195413.

Asahi, R., Y. Taga, W. Mannstadt, and A. J. Freeman (2000), Physical Review B 61 (11), 7459

Ast, C. R., and I. Gierz (2012), Physical Review B 86 (8), 085105 .

Ast, C. R., J. Henk, A. Ernst, L. Moreschini, M. C. Falub, D. Pacilé, P. Bruno, K. Kern, and M. Grioni (2007), Physical Review Letters 98 (18), 186807

Bahramy, M. S., R. Arita, and N. Nagaosa (2011), Physical Review B 84 (4), 041202.

Bahrdt, J., R. Follath, W. Frentrup, A. Gaupp, M. Scheer, R. Garrett, I. Gentle, K. Nugent, and S. Wilkins (2010), in AIP Conference Proceedings, Vol. 335, pp. 335-338.

Bena, C., and G. Montambaux (2009), New Journal of Physics 11 (9), 095003

Bentmann, H. (2012), Spin-Bahn-Kopplung in Grenzschichten: Mikroskopische Zusammenhänge und Strategien zur Manipulation, Ph.D. thesis (Universität Würzburg).

Berglund, C. N., and W. E. Spicer (1964), Physical Review 136 (4A), A1044

Berkebile, S., P. Puschnig, G. Koller, M. Oehzelt, F. P. Netzer, C. Ambrosch-Draxl, and M. G. Ramsey (2008), Physical Review B 77 (11), 115312

Bian, G., L. Zhang, Y. Liu, T. Miller, and T.-C. Chiang (2012), Physical Review Letters 108 (18), 186403

Bihlmayer, G., S. Blügel, and E. V. Chulkov (2007), Physical Review B 75 (19), 195414

Borstel, G., W. Braun, M. Neumann, and G. Seitz (1979), Physica Status Solidi (b) 95 (2), 453

Borstel, G., M. Neumann, and M. Wöhlecke (1981), Physical Review B 23 (7), 3121.

Bostwick, A., T. Ohta, T. Seyller, K. Horn, and E. Rotenberg (2007), Nature Physics 3 (1), 36

Bovensiepen, U., and P. Kirchmann (2012), Laser \& Photonics Reviews 6 (5), 589.

Bradshaw, A. M., and D. P. Woodruff (2015), New Journal

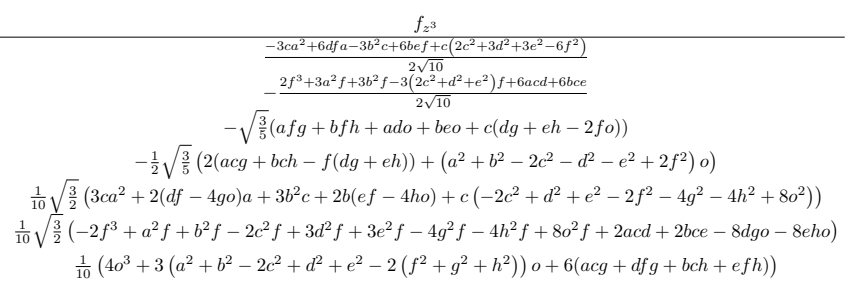

of Physics 17 (1), 013033

Braun, J. (1996), Reports on Progress in Physics 59 (10), 1267.

Braun, J., G. Borstel, and W. Nolting (1992), Physical Review B 46 (6), 3510

Braun, J., and M. Donath (2004), Journal of Physics: Condensed Matter 16 (26), S2539

Braun, J., J. Minár, S. Mankovsky, V. N. Strocov, N. B. Brookes, L. Plucinski, C. M. Schneider, C. S. Fadley, and H. Ebert (2013), Physical Review B 88 (20), 205409.

Braun, J., G. Thörner, and G. Borstel (1985), physica status solidi (b) 130 (2), 643

Brouder, C., G. Panati, M. Calandra, C. Mourougane, and N. Marzari (2007), Physical Review Letters 98 (4), 046402

Brundle, C. R., and A. D. Baker, Eds. (1978), Electron Spectroscopy: Theory, Techniques and Applications (Academic Press, New York/San Francisco/London, London, New York, San Francisco).

Cao, Y., J. a. Waugh, X.-W. X.-W. Zhang, J.-W. J.-W. Luo, Q. Wang, T. J. Reber, S. K. Mo, Z. Xu, a. Yang, J. Schneeloch, G. D. Gu, M. Brahlek, N. Bansal, S. Oh, a. Zunger, and D. S. Dessau (2013), Nature Physics 9 (8), 499, arXiv:arXiv:1011.1669v3.

Carlson, T. A. (1975), Annual Review of Physical Chemistry 26 (1), 211.

Castro Neto, A. H., F. Guinea, N. M. R. Peres, K. S. Novoselov, and A. K. Geim (2009), Reviews of Modern Physics 81 (1), 109

Chassé, A., and P. Rennert (1997), Physical Review B 55 (7), 4120

Chen, Y. L., J. G. Analytis, J.-H. Chu, Z. K. Liu, S.-K. Mo, X. L. Qi, H. J. Zhang, D. H. Lu, X. Dai, Z. Fang, S. C. Zhang, I. R. Fisher, Z. Hussain, and Z.-X. Shen (2009), Science 325 (5937), 178

Chuang, Y.-D. (2001), Science 292 (5521), 1509.

Condon, E. U., and G. H. Shortley (1935), The theory of atomic spectra (Cambridge University Press).

Cooper, J. W. (1962), Physical Review 128 (2), 681

Cotton, F. A. F. A. (1990), Pyrodynamic, 3rd ed. (John Wiley \& sons, New York, N.Y.).

Cowan, R. D. (1981), Los Alamos Series in Basic and Applied Sciences (University of California Press, Berkeley).

Crepaldi, A., L. Moreschini, G. Autès, C. Tournier-Colletta, S. Moser, N. Virk, H. Berger, P. Bugnon, Y. J. Chang, K. Kern, A. Bostwick, E. Rotenberg, O. V. Yazyev, and M. Grioni (2012), Physical Review Letters 109 (9), 096803 , arXiv:1205.1395

Daimon, H., T. Nakatani, S. Imada, S. Suga, Y. Kagoshima, and T. Miyahara (1993), Japanese Journal of Applied Physics 32 (Part 2, No. 10A), L1480

Damascelli, A. (2004), Physica Scripta T109, 61.

Damascelli, A., Z. Hussain, and Z.-X. Shen (2003), Reviews of Modern Physics 75 (2), 473 
Dauth, M., T. Körzdörfer, S. Kümmel, J. Ziroff, M. Wiessner, A. Schöll, F. Reinert, M. Arita, and K. Shimada (2011), Physical Review Letters 107 (19), 193002.

Dauth, M., M. Wiessner, V. Feyer, A. Schöll, P. Puschnig, F. Reinert, and S. Kümmel (2014), New Journal of Physics 16 (10), 103005.

Drake, G. W. F., and M. M. Cassar (2006), SciencesNew York, edited by G. Drake (Springer New York, New York, NY).

Eberhardt, W., and E. W. Plummer (1980), Physical Review B 21 (8), 3245

Eberly, D. (2008), Geometric Tools, LLC, Technical Report ,

1 .

Ebert, H., D. Ködderitzsch, and J. Minár (2011), Reports on

Progress in Physics 74 (9), 096501

Einstein, A. (1905), Annalen Der Physik 17 (6), 132.

Escher, M., N. Weber, M. Merkel, C. Ziethen, P. Bernhard, G. Schönhense, S. Schmidt, F. Forster, F. Reinert, B. Krömker, and D. Funnemann (2005), Journal of Physics: Condensed Matter 17 (16), S1329

Eskes, H., L. H. Tjeng, and G. A. Sawatzky (1990), Physical Review B 41 (1), 288.

Fadley, C. (2010), Journal of Electron Spectroscopy and Related Phenomena 178-179, 2

Fadley, C. S., and S. Nemšák (2014), Journal of Electron Spectroscopy and Related Phenomena 195, 409

Fanelsa, A., R. Schellenberg, F. U. Hillebrecht, E. Kisker, J. G. Menchero, A. P. Kaduwela, C. S. Fadley, and M. A. Van Hove (1996), Physical Review B 54 (24), 17962

Fano, U., and J. W. Cooper (1968), Reviews of Modern Physics 40 (3), 441

Fecher, G., V. Kuznetsov, N. Cherepkov, and G. Schönhense (2002), Journal of Electron Spectroscopy and Related Phenomena 122 (2), 157

Feder, R. (1981), Journal of Physics C: Solid State Physics 14 (15), 2049

Feder, R., and H. J. Meister (1969), Zeitschrift für Physik 229 (3-5), 309.

Feder, R., F. Rosicky, and B. Ackermann (1983), Zeitschrift für Physik B Condensed Matter 52 (1), 31

Feng, D. L., C. Kim, H. Eisaki, D. H. Lu, A. Damascelli, K. M. Shen, F. Ronning, N. P. Armitage, N. Kaneko, M. Greven, J.-i. Shimoyama, K. Kishio, R. Yoshizaki, G. D. Gu, and Z.-X. Shen (2002), Physical Review B 65 (22), 220501.

Finetti, P., D. Holland, C. Latimer, and C. Binns (2004), Nuclear Instruments and Methods in Physics Research Section B: Beam Interactions with Materials and Atoms 215 (3-4), 565 .

Frantzeskakis, E., and M. Grioni (2011), Physical Review B 84 (15), 155453.

Frantzeskakis, E., S. Pons, and M. Grioni (2010), Physical Review B 82 (8), 085440.

Frantzeskakis, E., S. Pons, H. Mirhosseini, J. Henk, C. R. Ast, and M. Grioni (2008), Physical Review Letters 101 (19), 196805 .

Gadzuk, J. (1974a), Solid State Communications 15 (6), 1011

Gadzuk, J. (1974b), Physical Review B 10 (12), 5030

Gadzuk, J. (1975), Physical Review B 12 (12), 5608.

Gelius, U., and K. Siegbahn (1972), Faraday Discussions of the Chemical Society 54, 257.

Geller, M. R., and W. Kohn (1993), Physical Review B 48 (19), 14085.

Ghijsen, J., L. H. Tjeng, J. van Elp, H. Eskes, J. Westerink, G. A. Sawatzky, and M. T. Czyzyk (1988), Physical Review
B 38 (16), 11322

Ghosh, a., and S. Karmakar (1999), The European Physical Journal B 11 (4), 575

Gierz, I., J. Henk, H. Höchst, C. R. Ast, and K. Kern (2011), Physical Review B 83 (12), 121408, arXiv:1010.1618.

Gierz, I., T. Suzuki, E. Frantzeskakis, S. Pons, S. Ostanin, A. Ernst, J. Henk, M. Grioni, K. Kern, and C. R. Ast (2009), Physical Review Letters 103 (4), 046803.

Gobeli, G. W., F. G. Allen, and E. O. Kane (1964), Physical Review Letters 12 (4), 94.

Goldberg, S., C. Fadley, and S. Kono (1981), Journal of Electron Spectroscopy and Related Phenomena 21 (4), 285

Goringe, C. M., D. R. Bowler, and E. Hernández (1997), Reports on Progress in Physics 60 (12), 1447.

Graber, T., F. Forster, A. Schöll, and F. Reinert (2011), Surface Science 605 (9-10), 878

Gray, a. X., J. Minár, S. Ueda, P. R. Stone, Y. Yamashita, J. Fujii, J. Braun, L. Plucinski, C. M. Schneider, G. Panaccione, H. Ebert, O. D. Dubon, K. Kobayashi, and C. S. Fadley (2012), Nature Materials 11 (11), 957.

Gray, a. X., C. Papp, S. Ueda, B. Balke, Y. Yamashita, L. Plucinski, J. Minár, J. Braun, E. R. Ylvisaker, C. M. Schneider, W. E. Pickett, H. Ebert, K. Kobayashi, and C. S. Fadley (2011), Nature Materials 10 (10), 759

Grobman, W. D. (1978), Physical Review B 17 (12), 4573

Gumbs, G., and M. K. Ali (1989), Journal of Physics A: Mathematical and General 22 (8), 951

Hallwachs, W. (1888), Annalen Der Physik 269, 301.

Hasan, M. Z., and C. L. Kane (2010), Reviews of Modern Physics 82 (4), 3045

Hengsberger, M., D. Purdie, P. Segovia, M. Garnier, and Y. Baer (1999), Physical Review Letters 83 (3), 592

Henk, J. (2002), in Handbook of Thin Films (Elsevier) pp. 479-526.

Henk, J., W. Schattke, H.-P. Barnscheidt, C. Janowitz, R. Manzke, and M. Skibowski (1989), Physical Review B 39 (18), 13286

Henk, J., W. Schattke, H. Cartensen, R. Manzke, and M. Skibowski (1993), Physical Review B 47 (4), 2251

Hermanson, J. (1993), Solid State Communications 88 (1112), 1097

Hertz, H. (1887), Annalen Der Physik 267, 983.

Himpsel, F. (1983), Advances in Physics 32 (1), 1

Hopkinson, J. F. L., and J. B. Pendry (1980), Computer Physics Communications 19 (1), 69

Hsieh, D., D. Qian, L. Wray, Y. Xia, Y. S. Hor, R. J. Cava, and M. Z. Hasan (2008), Nature 452 (7190), 970

Hüfner, S. (1995), Photoelectron spectroscopy. Principles and applications, 2nd ed. (Springer, Berlin/Heidelberg/New York).

Hüfner, S. (2007), Lecture Notes in Physics, 1st ed. (Springer, Berlin/Heidelberg/New York).

Hussain, Z., S. Kono, R. E. Connelly, and C. S. Fadley (1980), Physical Review Letters 44 (13), 895.

Hwang, C., C.-H. Park, D. A. Siegel, A. V. Fedorov, S. G. Louie, and A. Lanzara (2011), Physical Review B 84 (12), 125422 (10 pp.).

Inosov, D. S., J. Fink, A. A. Kordyuk, S. V. Borisenko, V. B. Zabolotnyy, R. Schuster, M. Knupfer, B. Büchner, R. Follath, H. A. Dürr, W. Eberhardt, V. Hinkov, B. Keimer, and H. Berger (2007), Physical Review Letters 99 (23), 237002

Ishizaka, K., M. S. Bahramy, H. Murakawa, M. Sakano, T. Shimojima, T. Sonobe, K. Koizumi, S. Shin, H. Miya- 
hara, A. Kimura, K. Miyamoto, T. Okuda, H. Namatame, M. Taniguchi, R. Arita, N. Nagaosa, K. Kobayashi, Y. Murakami, R. Kumai, Y. Kaneko, Y. Onose, and Y. Tokura (2011), Nature Materials 10 (7), 521.

Jahne, E., and E. Gutsche (1967), physica status solidi (b) $21(1), 57$.

Jahnke, T., T. Weber, A. L. Landers, A. Knapp, S. Schössler, J. Nickles, S. Kammer, O. Jagutzki, L. Schmidt, A. Czasch, T. Osipov, E. Arenholz, A. T. Young, R. Díez Muiño, D. Rolles, F. J. García de Abajo, C. S. Fadley, M. A. Van Hove, S. K. Semenov, N. A. Cherepkov, J. Rösch, M. H. Prior, H. Schmidt-Böcking, C. L. Cocke, and R. Dörner (2002), Physical Review Letters 88 (7), 073002

Jozwiak, C., C.-H. Park, K. Gotlieb, C. Hwang, D.-H. Lee, S. G. Louie, J. D. Denlinger, C. R. Rotundu, R. J. Birgeneau, Z. Hussain, and A. Lanzara (2013), Nature Physics 9 (5), 293 , arXiv:1302.5094.

Jung, W. S., C. S. Leem, C. Kim, S. R. Park, S. Y. Park, B. J. Kim, E. Rotenberg, and C. Kim (2010), Physical Review B 82 (23), 235105

Kane, C. L. (2008), Nature 4, 348.

Kane, C. L., and E. J. Mele (2005), Physical Review Letters 95 (14), 146802.

Kane, C. L., and E. J. Mele (2006), Science 314 (5806), 1692

Kera, S., S. Tanaka, H. Yamane, D. Yoshimura, K. K. Okudaira, K. Seki, and N. Ueno (2006), Chemical Physics 325 (1), 113

Kevan, S. D. (1992), Angle-Resolved Photoemission: Theory and Current Applications (Elsevier, Amsterdam).

Kim, B. J., H. Jin, S. J. Moon, J. . Y. Kim, B. . G. Park, C. S. Leem, J. Yu, T. W. Noh, C. Kim, S. . J. Oh, J. . H. Park, V. Durairaj, G. Cao, and E. Rotenberg (2008), Physical Review Letters 101 (7), 76402

Kim, B. J., H. Ohsumi, T. Komesu, S. Sakai, T. Morita, H. Takagi, and T. Arima (2009), Science 323 (5919), 1329

King, P. D. C., R. H. He, T. Eknapakul, P. Buaphet, S.-K. Mo, Y. Kaneko, S. Harashima, Y. Hikita, M. S. Bahramy, C. Bell, Z. Hussain, Y. Tokura, Z.-X. Shen, H. Y. Hwang, F. Baumberger, and W. Meevasana (2012), Physical Review Letters 108 (11), 117602

Kittel, C. (1996), Introduction to solid state physics, 5th edition, 7th ed. (John Wiley, New York, NY).

Koide, T., T. Shidara, and M. Yuri (1993), Nuclear Instruments and Methods in Physics Research Section A: Accelerators, Spectrometers, Detectors and Associated Equipment 336 (1-2), 368

Koide, T., T. Shidara, M. Yuri, N. Kandaka, K. Yamaguchi, and H. Fukutani (1991), Nuclear Instruments and Methods in Physics Research Section A: Accelerators, Spectrometers, Detectors and Associated Equipment 308 (3), 635.

Koller, G., S. Berkebile, M. Oehzelt, P. Puschnig, C. Ambrosch-Draxl, F. P. Netzer, and M. G. Ramsey (2007), Science 317 (5836), 351.

König, M., S. Wiedmann, C. Brune, A. Roth, H. Buhmann, L. W. Molenkamp, X.-L. Qi, and S.-C. Zhang (2007), Science 318 (5851), 766

Koster, G. F., and J. C. Slater (1954), Physical Review 95 (5), 1167.

Krömker, B., M. Escher, D. Funnemann, D. Hartung, H. Engelhard, and J. Kirschner (2008), Review of Scientific Instruments 79 (5), 53702

Lanzara, A., P. V. Bogdanov, X. J. Zhou, S. A. Kellar, D. L. Feng, E. D. Lu, T. Yoshida, H. Eisaki, A. Fujimori, K. Kishio, J. I. Shimoyama, T. Noda, S. Uchida, Z. Hus- sain, and Z. X. Shen (2001), Nature 412 (6846), 510

Larsson, C. G., and J. B. Pendry (1981), Journal of Physics C: Solid State Physics 14 (21), 3089

LaShell, S., E. Jensen, and T. Balasubramanian (2000), Physical Review B 61 (3), 2371.

Lenard, P. (1900), Annalen Der Physik 2 (6), 359.

Lew Yan Voon, L. C., M. Willatzen, M. Cardona, and N. E. Christensen (1996), Physical Review B 53 (16), 10703.

Liebsch, A. (1974), Physical Review Letters 32 (21), 1203

Liebsch, A. (1976), Physical Review B 13 (2), 544

Liebsch, A., and E. W. Plummer (1974), Faraday Discussions 58,19

Liu, C.-X., X.-L. Qi, H. Zhang, X. Dai, Z. Fang, and S.-C. Zhang (2010), Physical Review B 82 (4), 45122

Liu, G., Z. Wang, and S.-S. Li (2009), Physics Letters A 373 (23-24), 2091

Liu, Y., G. Bian, T. Miller, and T.-C. Chiang (2011), Physical Review Letters 107 (16), 166803

Liu, Y., D. Ikeda, S. Nagamatsu, T. Nishi, N. Ueno, and S. Kera (2014), Journal of Electron Spectroscopy and Related Phenomena 195, 287.

Louie S. G., S. G., P. Thiry, R. Pinchaux, Y. Petroff, D. Chandesris, and J. Lecante (1980), Physical Review Letters $44(8), 549$.

Lueftner, D., T. Ules, E. M. Reinisch, G. Koller, S. Soubatch, F. S. Tautz, M. G. Ramsey, and P. Puschnig (2014), Proceedings of the National Academy of Sciences 111 (2), 605.

Mahan, G. D. (1970), Physical Review B 2 (11), 4334

Mahan, G. D. (1993), Many-Particle Physics, 2nd ed. (Plenum, New York, N.Y.).

Mao, S., A. Yamakage, and Y. Kuramoto (2011), Physical Review B 84 (11), 115413.

Marzari, N., A. A. Mostofi, J. R. Yates, I. Souza, and D. Vanderbilt (2012), Reviews of Modern Physics 84 (4), 1419.

Meevasana, W., P. D. C. King, R. H. He, S.-K. Mo, M. Hashimoto, A. Tamai, P. Songsiriritthigul, F. Baumberger, and Z.-X. Shen (2011), Nature Materials 10 (2), 114

Meier, F., H. Dil, J. Lobo-Checa, L. Patthey, and J. Osterwalder (2008), Physical Review B 77 (8), 165431

Meier, F., J. H. Dil, and J. Osterwalder (2009), New Journal of Physics 11 (12), 125008.

Mii, T., N. Shima, K. Kano, and K. Makoshi (2014), Journal of the Physical Society of Japan 83 (6), 64706.

Minár, J. (2011), Journal of Physics: Condensed Matter 23 (25), 253201.

Minár, J. (2014), in DMFT at 25: Infinite Dimensions. Lecture Notes of the Autumn School on Correlated Electrons, edited by E. Pavarini, E. Koch, D. Vollhardt, and A. Lichtenstein, 4th ed., Chap. 13 (Forschungszentrum Julich).

Minár, J., J. Braun, S. Mankovsky, and H. Ebert (2011), Journal of Electron Spectroscopy and Related Phenomena 184 (3), 91

Moreschini, L., S. Moser, A. Ebrahimi, B. Dalla Piazza, K. S. Kim, S. Boseggia, D. F. McMorrow, H. M. Ronnow, J. Chang, D. Prabhakaran, A. T. Boothroyd, E. Rotenberg, A. Bostwick, and M. Grioni (2014), Physical Review B 89 (20), 201114

Moser, S., L. Moreschini, A. Ebrahimi, B. Dalla Piazza, M. Isobe, H. Okabe, J. Akimitsu, V. V. Mazurenko, K. S. Kim, A. Bostwick, E. Rotenberg, J. Chang, H. M. Rønnow, and M. Grioni (2014a), New Journal of Physics 16 (1), 013008

Moser, S., L. Moreschini, J. Jaćimović, O. S. Barišić, 
H. Berger, A. Magrez, Y. J. Chang, K. S. Kim, A. Bostwick, E. Rotenberg, L. Forró, and M. Grioni (2013), Physical Review Letters 110 (19), 196403

Moser, S., L. Moreschini, H.-Y. Yang, D. Innocenti, F. Fuchs, N. H. Hansen, Y. J. Chang, K. S. Kim, A. L. Walter, A. Bostwick, E. Rotenberg, F. Mila, and M. Grioni (2014b), Physical Review Letters 113 (18), 187001

Mucha-Kruczyński, M., O. Tsyplyatyev, A. Grishin, E. McCann, V. I. Fal'ko, A. Bostwick, and E. Rotenberg (2008), Physical Review B 77 (19), 195403, arXiv:0711.1129

Mugarza, A., J. E. Ortega, F. J. Himpsel, and F. J. G. de Abajo (2003), Physical Review B 67 (8), 81404

Mugarza, A., J. E. Ortega, A. Mascaraque, E. G. Michel, K. N. Altmann, and F. J. Himpsel (2000), Physical Review B 62 (19), 12672

Nagano, M., A. Kodama, T. Shishidou, and T. Oguchi (2009), Journal of Physics-condensed Matter 21 (6), Minist Educ, Culture, Sports, Sci \& Technol Japan.

Nahon, L., and C. Alcaraz (2004), Applied Optics 43 (5), 1024 .

Nahon, L., N. de Oliveira, G. A. Garcia, J.-F. Gil, B. Pilette, O. Marcouillé, B. Lagarde, and F. Polack (2012), Journal of Synchrotron Radiation 19 (4), 508.

Nemšák, S., N. Kaduwela, and C. Fadley (2012), "Spatially resolved photo-electron cross-sections."

Nguyen, N. L., G. Borghi, A. Ferretti, I. Dabo, and N. Marzari (2015), Physical Review Letters 114 (16), 166405 .

Nordling, C., E. Sokolowski, and K. Siegbahn (1957), Physical Review 105 (5), 1676.

Noschese, S., L. Pasquini, and L. Reichel (2013), Numerical Linear Algebra with Applications 20 (2), 302.

Ohta, T., A. Bostwick, J. L. McChesney, T. Seyller, K. Horn, and E. Rotenberg (2007), Physical Review Letters 98 (20), 206802 .

Osterwalder, J., T. Greber, P. Aebi, R. Fasel, and L. Schlapbach (1996), Physical Review B 53 (15), 10209

Panati, G., and A. Pisante (2013), Communications in Mathematical Physics 322 (3), 835.

Papp, C., L. Plucinski, J. Minar, J. Braun, H. Ebert, C. M. Schneider, and C. S. Fadley (2011), Physical Review B 84 (4), 045433.

Park, C.-H., and S. G. Louie (2012), Physical Review Letters 109 (9), 097601

Park, S. R., J. Han, C. Kim, Y. Y. Koh, C. Kim, H. Lee, H. J. Choi, J. H. Han, K. D. Lee, N. J. Hur, M. Arita, K. Shimada, H. Namatame, and M. Taniguchi (2012), Physical Review Letters 108 (4), 046805.

Pendry, J. B. (1976), Surface Science 57 (2), 679.

Petersen, L., and P. Hedegard (2000), Surface Science 459 (12), 49

Plucinski, L., J. Minár, B. C. Sell, J. Braun, H. Ebert, C. M. Schneider, and C. S. Fadley (2008), Physical Review B 78 (3), 035108 .

Plummer, E. W., and W. Eberhardt (1982), Adv. Chem. Phys. 49, 533.

Podolsky, B., and L. Pauling (1929), Physical Review 34 (1), 109.

Premper, J., M. Trautmann, J. Henk, and P. Bruno (2007), Physical Review B 76 (7), 73310.

Puschnig, P., S. Berkebile, A. J. Fleming, G. Koller, K. Emtsev, T. Seyller, J. D. Riley, C. Ambrosch-Draxl, F. P. Netzer, and M. G. Ramsey (2009), Science 326 (5953), 702

Puschnig, P., and D. Lueftner (2015), Journal of Electron
Spectroscopy and Related Phenomena 200, 193.

Puschnig, P., E.-M. Reinisch, T. Ules, G. Koller, S. Soubatch, M. Ostler, L. Romaner, F. S. Tautz, C. Ambrosch-Draxl, and M. G. Ramsey (2011), Physical Review B 84 (23), 235427

Rotenberg, E. (2010), "Many-Body Interactions in Nanoscale Materials by Angle-Resolved Photoemission Spectroscopy," in X-Rays in Nanoscience, edited by G. J., Chap. ManyBody (Wiley-VCH Verlag GmbH \& Co. KGaA, Weinheim, Germany) pp. 169-209.

Rotenberg, E., and S. D. Kevan (2002), Journal of Electron Spectroscopy and Related Phenomena 126 (1-3), PII S0368.

Rotenberg, E., W. Theis, K. Horn, and P. Gille (2000), Nature 406 (6796), 602

S. A. Ketabi,, and N. Shahtahmasebi (2005), Iranian Journal of Physics Research 4 (1), 1.

Saito, T., K. Ozaki, K. Fukui, H. Iwai, K. Yamamoto, H. Miyake, and K. Hiramatsu (2014), Thin Solid Films 571 (P3), 517

Sánchez-Barriga, J., A. Varykhalov, J. Braun, S.-Y. Xu, N. Alidoust, O. Kornilov, J. Minár, K. Hummer, G. Springholz, G. Bauer, R. Schumann, L. V. Yashina, H. Ebert, M. Z. Hasan, and O. Rader (2014), Physical Review X 4 (1), 011046.

Sanderson, R. T. (1976), Chemical Bonds and Bond Energy, 2nd ed. (Academic Press, New York/San Francisco/London).

Sanderson, R. T. (1983), Chemical \& Engineering News, 2nd ed. (Academic Press, New York/San Francisco/London).

Santander-Syro, A. F., O. Copie, T. Kondo, F. Fortuna, S. Pailhès, R. Weht, X. G. Qiu, F. Bertran, A. Nicolaou, A. Taleb-Ibrahimi, P. Le Fèvre, G. Herranz, M. Bibes, N. Reyren, Y. Apertet, P. Lecoeur, A. Barthélémy, and M. J. Rozenberg (2011), Nature 469 (7329), 189.

Schaich, W. L., and N. W. Ashcroft (1971), Physical Review B 3 (8), 2452.

Schoenhense, G., K. Medjanik, H.-J. J. Elmers, G. Schönhense, K. Medjanik, and H.-J. J. Elmers (2015), Journal of Electron Spectroscopy and Related Phenomena 200, 94.

Schönhense, G. (1990), Physica Scripta T31, 255.

Seah, M. P., and W. A. Dench (1979), Surface and Interface Analysis 1 (1), 2 .

Shanavas, K. V., Z. S. Popović, and S. Satpathy (2014), Physical Review B 90 (16), 165108

Shi, J., S. . J. Tang, B. Wu, P. T. Sprunger, W. L. Yang, V. Brouet, X. J. Zhou, Z. Hussain, Z. . X. Shen, Z. Zhang, and E. W. Plummer (2004), Physical Review Letters 92 (18), 186401/1

Shinotsuka, H., S. Tanuma, C. J. Powell, and D. R. Penn (2015), Surface and Interface Analysis 47 (9), 871.

Shirley, D. A. (1972), Physical Review B 5 (12), 4709.

Shirley, E. L., L. J. Terminello, A. Santoni, and F. J. Himpsel (1995), Physical Review B 51 (19), 13614

Smallwood, C. L., C. Jozwiak, W. Zhang, and A. Lanzara (2012), Review of Scientific Instruments 83 (12), 123904.

Sólyom, J. (2007), Fundamentals of the physics of solids: volume 1 - structure and dynamics (Springer, Berlin/Heidelberg/New York).

Spanjaard, D. J., D. W. Jepsen, and P. M. Marcus (1977), Physical Review B 15 (4), 1728

Stewart, J. J. P. (2007), "Generic (non-trigonometric) rotation of atomic orbitals," 
Stolow, A., A. E. Bragg, and D. M. Neumark (2004), Chemical Reviews 104 (4), 1719

Tanuma, S., C. Powell, and D. Penn (1987), Surface Science Letters 192 (1), L849.

Tanuma, S., C. J. Powell, and D. R. Penn (1988), Surface and Interface Analysis 11 (11), 577.

Thörner, G., and G. Borstel (1984), Physica Status Solidi B-basic Research 126 (2), 617.

Tougaard, S. (1990), Journal of Vacuum Science \& Technology A-vacuum Surfaces and Films 8 (3), AMER VACUUM SOC.

Turner, N. H. (1988), Analytical Chemistry 60 (12), 377.

Tusche, C., M. Ellguth, A. A. Uenal, C. . T. Chiang, A. Winkelmann, A. Krasyuk, M. Hahn, G. Schoenhense, and J. Kirschner (2011), Applied Physics Letters 99 (3), 32505

Ueno, N., A. Kitamura, K. K. Okudaira, T. Miyamae, Y. Harada, S. Hasegawa, H. Ishii, H. Inokuchi, T. Fujikawa, T. Miyazaki, and K. Seki (1997), The Journal of Chemical Physics 107 (6), 2079.

Ueno, N., K. Suzuki, S. Hasegawa, K. Kamiya, K. Seki, and H. Inokuchi (1993), The Journal of Chemical Physics $99(9), 7169$.

Uschakow, S., A. Gaupp, M. MacDonald, and F. Schäfers (2013), Journal of Physics: Conference Series 425 (15), 152011

Valla, T., A. V. Fedorov, P. D. Johnson, and S. L. Hulbert (1999a), Physical Review Letters 83 (10), 2085.

Valla, T., A. V. Fedorov, P. D. Johnson, B. O. Wells, S. L. Hulbert, Q. Li, G. D. Gu, and N. Koshizuka (1999b), Science 285 (5436), 2110

Vasilyev, D., C. Tusche, F. Giebels, H. Gollisch, R. Feder, and J. Kirschner (2015), Journal of Electron Spectroscopy and Related Phenomena 199, 10

Wagner, L., Z. Hussain, C. Fadley, and R. Baird (1977), Solid State Communications 21 (5), 453.

Wang, X.-P., P. Richard, Y.-B. Huang, H. Miao, L. Cevey, N. Xu, Y.-J. Sun, T. Qian, Y.-M. Xu, M. Shi, J.-P. Hu, X. Dai, and H. Ding (2012), Physical Review B 85 (21), 214518 .

Wang, Y. H., D. Hsieh, D. Pilon, L. Fu, D. R. Gardner, Y. S. Lee, N. Gedik, I. Si, S. I. Ii, and V. Si (2011), Physical Review Letters 107 (20), 1, arXiv:arXiv:1101.5636v2.

Wannier, G. H. (1937), Physical Review 52 (3), 191.

Weiß, S., D. Lüftner, T. Ules, E. M. Reinisch, H. Kaser, A. Gottwald, M. Richter, S. Soubatch, G. Koller, M. G. Ramsey, F. S. Tautz, and P. Puschnig (2015), Nature Communications 6, 8287.

Westphal, C., J. Bansmann, M. Getzlaff, and G. Schönhense (1989), Physical Review Letters 63 (2), 151.

White, R., C. Fadley, M. Sagurton, P. Roubin, D. Chandesris, J. Lecante, C. Guillot, and Z. Hussain (1987), Physical Review B 35 (3), 1147

Wießner, M., D. Hauschild, C. Sauer, V. Feyer, A. Schöll, and F. Reinert (2014), Nature Communications 5, 4156.

Wiessner, M., D. Hauschild, A. Schoell, F. Reinert, V. Feyer, K. Winkler, and B. Kroemker (2012), Physical Review B 86 (4), 45417.

Wöhlecke, M., and G. Borstel (1983), Physica Scripta T4 (T4), 162

Wöhlecke, M., and G. Borstel (1984), in Optical Orientation, edited by F. Meier and B. Zakharchenya, 1st ed. (NorthHolland, Amsterdam) pp. 423-462.

Woicik, J. C. (2016), Hard X-ray Photoelectron Spectroscopy
(HAXPES), edited by J. C. Woicik, Springer Series in Surface Sciences, Vol. 59 (Springer International Publishing, Cham).

Wolfram Research, I. (2016), Mathematica, version 10 ed. (Wolfram Research, Inc., Champaign, Illinois).

Xia, Y., D. Qian, D. Hsieh, L. Wray, A. Pal, H. Lin, A. Bansil, D. Grauer, Y. S. Hor, R. J. Cava, and M. Z. Hasan (2009), Nature Physics 5 (6), 398.

Xie, Z., S. He, C. Chen, Y. Feng, H. Yi, A. Liang, L. Zhao, D. Mou, J. He, Y. Peng, X. Liu, Y. Liu, G. Liu, X. Dong, L. Yu, J. Zhang, S. Zhang, Z. Wang, F. Zhang, F. Yang, Q. Peng, X. Wang, C. Chen, Z. Xu, and X. J. Zhou (2014), Nature Communications 5 (May 2013), 3382 , arXiv:1303.0698

Yamamoto, S., Y. Senba, T. Tanaka, H. Ohashi, T. Hirono, H. Kimura, M. Fujisawa, J. Miyawaki, A. Harasawa, T. Seike, S. Takahashi, N. Nariyama, T. Matsushita, M. Takeuchi, T. Ohata, Y. Furukawa, K. Takeshita, S. Goto, Y. Harada, S. Shin, H. Kitamura, A. Kakizaki, M. Oshima, and I. Matsuda (2014), Journal of Synchrotron Radiation 21 (2), 352.

Zaanen, J., G. A. Sawatzky, and J. W. Allen (1985), Physical Review Letters 55 (4), 418.

Zhang, F. C., and T. M. Rice (1988), Physical Review B 37 (7), 3759.

Zhang, H., C.-X. Liu, X.-L. Qi, X. Dai, Z. Fang, and S.-C. Zhang (2009), Nature Physics 5 (6), 438.

Zhang, H., C.-X. Liu, and S.-C. Zhang (2013), Physical Review Letters 111 (6), 066801 (5 pp.).

Zhong, J. X., J. Q. You, J. R. Yan, and X. H. Yan (1991), Physical Review B 43 (16), 13778.

Zhu, Z. . H., C. N. Veenstra, S. Zhdanovich, M. P. Schneider, T. Okuda, K. Miyamoto, S. . Y. Zhu, H. Namatame, M. Taniguchi, M. W. Haverkort, I. S. Elfimov, and A. Damascelli (2014), Physical Review Letters 112 (7), 76802

Zhu, Z.-H., C. N. Veenstra, G. Levy, A. Ubaldini, P. Syers, N. P. Butch, J. Paglione, M. W. Haverkort, I. S. Elfimov, and A. Damascelli (2013), Physical Review Letters 110 (21), 216401.

Ziroff, J., F. Forster, A. Schoell, P. Puschnig, and F. Reinert (2010), Physical Review Letters 104 (23), 233004. 